\title{
CONGRUENCE MODULES RELATED TO EISENSTEIN SERIES
}

\author{
BY MASAMI OHTA
}

ABSTRACT. - The purpose of this paper is to study the structure of congruence modules (or modules of congruences) associated with Eisenstein series in various contexts in the $\Lambda$-adic theory of elliptic modular forms. Under some assumptions, we explicitly describe such modules in terms of Kubota-Leopoldt $p$-adic $L$-functions.

() 2003 Éditions scientifiques et médicales Elsevier SAS

RÉSUMÉ. - Le but de cet article est d'étudier la structure des modules de congruence qui, dans la théorie $\Lambda$-adique des formes modulaires elliptiques, sont, de diverses manières, associées aux séries d'Eisenstein. Sous certaines conditions, nous décrivons explicitement de tels modules en termes des fonctions $L$ $p$-adiques de Kubota-Leopoldt.

(c) 2003 Éditions scientifiques et médicales Elsevier SAS

\section{Introduction}

The notion of congruence modules (or modules of congruences) was introduced by Hida [4,5] to study congruence properties of modular forms in terms of Hecke algebras. In the works of Hida, such modules are thus defined for rings, but one can consider similar modules replacing rings by modules in an obvious way. For our purpose, it is convenient to formulate it in the following manner: Let $R$ be an integral domain with its quotient field $L$. We consider an exact sequence of flat $R$-modules:

$$
0 \rightarrow A \stackrel{i}{\rightarrow} B \stackrel{\pi}{\rightarrow} C \rightarrow 0
$$

given with its splitting over $L$, i.e. when tensored with $L$ over $R$ :

$$
0 \leftarrow A \otimes_{R} L \stackrel{t}{\leftarrow} B \otimes_{R} L \stackrel{s}{\leftarrow} C \otimes_{R} L \leftarrow 0 .
$$

The congruence module attached to this situation is defined by

$$
\mathcal{C}:=C / \pi(B \cap s(C)) \cong t(B) / A \text {. }
$$

The aim of this paper is to study the structure of such modules, which are naturally associated with Eisenstein series in various contexts.

Since the exact statement of our main result requires rather lengthy preparation of terminologies, we would like to first illustrate what kind of problems we are going to consider, in the following elementary situation: 
Let $\Gamma=S L_{2}(\mathbf{Z})$, and denote by $M_{k}(\Gamma)$ (resp. $S_{k}(\Gamma)$ ) the space of holomorphic modular forms (resp. cusp forms) of weight $k$ with respect to $\Gamma$, for $k \geqslant 4$ even. Via the $q$-expansion at the unique cusp $i \infty$ of $\Gamma$, we consider this space as embedded in $\mathbf{C}[[q]]$ and set:

$$
\begin{cases}M_{k}(\Gamma ; \mathbf{Z}):=M_{k}(\Gamma) \cap \mathbf{Z} \llbracket q \rrbracket & \text { and } M_{k}(\Gamma ; R):=M_{k}(\Gamma ; \mathbf{Z}) \otimes \mathbf{Z} R, \\ S_{k}(\Gamma ; \mathbf{Z}):=S_{k}(\Gamma) \cap \mathbf{Z} \llbracket q \rrbracket & \text { and } S_{k}(\Gamma ; R):=S_{k}(\Gamma ; \mathbf{Z}) \otimes \mathbf{Z} R,\end{cases}
$$

for any ring $R$. Let $p \geqslant 5$ be a prime number, and assume that $k \not \equiv 0 \bmod (p-1)$. We take $\mathbf{Z}_{p}$ for $R$. It is easy to see that we have an exact sequence:

$$
0 \rightarrow S_{k}\left(\Gamma ; \mathbf{Z}_{p}\right) \rightarrow M_{k}\left(\Gamma ; \mathbf{Z}_{p}\right) \stackrel{\pi}{\rightarrow} \mathbf{Z}_{p} \rightarrow 0
$$

where $\pi$ sends an element of $M_{k}\left(\Gamma ; \mathbf{Z}_{p}\right)\left(\hookrightarrow \mathbf{Z}_{p} \llbracket q \rrbracket\right)$ to its constant term. Let $\mathcal{H}$ (resp. $h$ ) be the $\mathbf{Z}_{p}$-algebra generated by the standard Hecke operators acting on $M_{k}\left(\Gamma ; \mathbf{Z}_{p}\right)\left(\right.$ resp. $S_{k}\left(\Gamma ; \mathbf{Z}_{p}\right)$ ). Then the exact sequence (i) splits uniquely as $\mathcal{H}$-modules over $\mathbf{Q}_{p}$, giving $t$ and $s$ as above. In this case, the associated congruence module $\mathcal{C}=\mathbf{Z}_{p} / \pi\left(M_{k}\left(\Gamma ; \mathbf{Z}_{p}\right) \cap s\left(\mathbf{Q}_{p}\right)\right)$ is quite easy to describe: Let

$$
E_{k}=\frac{\zeta(1-k)}{2}+\sum_{n=1}^{\infty}\left(\sum_{0<d \mid n} d^{k-1}\right) q^{n} \in M_{k}(\Gamma ; \mathbf{Q})
$$

be the usual Eisenstein series, where $\zeta$ is Riemann's zeta function, and note that

$$
\zeta(1-k) / 2=-B_{k} / 2 k \in \mathbf{Q}
$$

is $p$-integral. Then it is clear that $M_{k}\left(\Gamma ; \mathbf{Z}_{p}\right) \cap s\left(\mathbf{Q}_{p}\right)$ is the $\mathbf{Z}_{p}$-module generated by $E_{k}$; and hence $\mathcal{C} \cong \mathbf{Z}_{p} / \zeta(1-k) \mathbf{Z}_{p}$.

Next, let us define the Eisenstein ideal of $\mathcal{H}$ (resp. $h$ ) by $\mathcal{I}:=\operatorname{Ann}_{\mathcal{H}}\left(E_{k}\right)$ (resp. $I:=$ (the image of $\mathcal{I}$ by $H \rightarrow h$ )). The module $\mathcal{C}$ above is an $\mathcal{H}$-module in an obvious manner, and moreover the action of $\mathcal{H}$ factors through $\mathcal{H} \rightarrow \mathcal{H} / \mathcal{I} \rightarrow h / I$. As a consequence, we have a surjective ring homomorphism $h / I \rightarrow \mathbf{Z}_{p} / \zeta(1-k) \mathbf{Z}_{p}$. Here, the left-hand side may be interpreted as the congruence module attached to the exact sequence

$$
0 \rightarrow \mathcal{I} \rightarrow \mathcal{H} \rightarrow \mathbf{Z}_{p} \rightarrow 0
$$

together with its unique splitting as $\mathcal{H}$-modules over $\mathbf{Q}_{p}$. It is not difficult, but less trivial than the case (i), to show that the surjection above is in fact an isomorphism (cf. Kurihara [9], Lemma 3.1).

A similar problem arises for the Eichler-Shimura cohomology groups: Let

$$
S^{k-2}\left(\mathbf{Z}_{p}\right)=\mathbf{Z}_{p}^{\oplus(k-1)}
$$

on which $\Gamma$ acts via the symmetric tensor representation of degree $k-2$. Then we have an exact sequence of free $\mathbf{Z}_{p}$-modules, considered by Harder and Pink [2]:

$$
0 \rightarrow H_{P}^{1}\left(\Gamma, S^{k-2}\left(\mathbf{Z}_{p}\right)\right)_{\mathrm{Eis}} \rightarrow H^{1}\left(\Gamma, S^{k-2}\left(\mathbf{Z}_{p}\right)\right)_{\mathrm{Eis}} \rightarrow \mathbf{Z}_{p} \rightarrow 0 .
$$

Here, $H_{P}^{1}$ is the first parabolic cohomology, and the subscript "Eis" means the localization at the maximal ideal $(\mathcal{I}, p)$ of $\mathcal{H}$. The Eichler-Shimura theory implies that the sequence (iii) uniquely splits over $\mathbf{Q}_{p}$ as $\mathcal{H}_{\text {Eis }}$-modules. In this case, the associated congruence module is again isomorphic to $\mathbf{Z}_{p} / \zeta(1-k) \mathbf{Z}_{p}$. However, this determination of the congruence module (or 
the "denominator of the Eisenstein cohomology class"), due to Harder, is by no means trivial, and it was one of the starting point of the investigation by Harder and Pink.

One can also ask about the structure of the congruence module associated with the exact sequence with canonical splitting over $\mathbf{Q}_{p}$ :

$$
0 \rightarrow \mathbf{Z}_{p} \rightarrow \operatorname{Symb}_{\Gamma}\left(S^{k-2}\left(\mathbf{Z}_{p}\right)\right)_{\mathrm{Eis}} \rightarrow H_{P}^{1}\left(\Gamma, S^{k-2}\left(\mathbf{Z}_{p}\right)\right)_{\mathrm{Eis}} \rightarrow 0
$$

where $\operatorname{Symb}_{\Gamma}\left(S^{k-2}\left(\mathbf{Z}_{p}\right)\right)$ is the group of $S^{k-2}\left(\mathbf{Z}_{p}\right)$-valued modular symbols over $\Gamma$ (cf. Greenberg and Stevens, [1], Theorem (4.3)). Once again, the answer is given by $\mathbf{Z}_{p} / \zeta(1-k) \mathbf{Z}_{p}$. This in fact follows from the above result of Harder, together with the Poincaré duality and (1.1.12) in the text.

The purpose of this paper is to consider similar problems, replacing $\mathbf{Z}_{p}$ by the Iwasawa algebra $\Lambda_{\mathfrak{r}}$ over the ring of integers $\mathfrak{r}$ of a finite extension of $\mathbf{Q}_{p}$, and the above classical objects by the corresponding $\Lambda$-adic ones. For this, we fix a positive integer $N$ prime to $p$. For each pair of Dirichlet characters $(\theta, \psi)$ such that the product of their conductors is either $N$ or $N p, \theta \psi$ is even, and the conductor of $\psi$ is prime to $p$, we can associate an ordinary (in the sense of Hida) $\Lambda$-adic Eisenstein series $\mathcal{E}(\theta, \psi)$ of level $N$. We can then consider the Eisenstein ideal and the (unique) maximal ideal containing it (the Eisenstein maximal ideal), in Hida's universal ordinary $p$-adic Hecke algebra acting on the space of ordinary $\Lambda$-adic modular forms of level $N$. Also, we have the Hecke algebra acting on the cohomology groups in (III) and (IV) below, which is canonically isomorphic to the above one. (But in the text, we always put the superscript "**" to this algebra and the Hecke operators in it, to indicate that we are considering the covariant action of Hecke correspondences, as in our previous works.) We have the corresponding Eisenstein (maximal) ideal in this algebra.

Now our objectives of study are the exact sequences of free $\Lambda_{\mathfrak{r}}$-modules replacing the objects in (i)-(iv) by the following $\Lambda$-adic ones of level $N$ localized at the above mentioned Eisenstein maximal ideals, respectively:

(I) The spaces of ordinary $\Lambda$-adic cusp forms and modular forms;

(II) Hida's ordinary $p$-adic Hecke algebra and its Eisenstein ideal;

(III) Ordinary $p$-adic Eichler-Shimura cohomology group and the ordinary generalized $p$-adic Eichler-Shimura cohomology group studied in our previous works;

(IV) The group of ordinary $p$-adic modular symbols and the ordinary $p$-adic Eichler-Shimura cohomology group.

All such exact sequences uniquely split over the quotient field of the Iwasawa algebra as modules over the Hecke algebras. Our main result of this paper is Theorem (1.5.5) which determines the precise structure of the congruence modules associated with the situations (I)-(IV) under the following assumptions:

$$
\left\{\begin{array}{l}
p \text { does not divide } \varphi(N) \\
\left(\theta \omega \psi^{-1}\right)(p) \neq 1
\end{array}\right.
$$

where $\varphi$ is the Euler function, and $\omega$ is the Teichmüller character. Indeed, the congruence modules are all isomorphic, and described in terms of Iwasawa's power series giving the KubotaLeopoldt $p$-adic $L$-function attached to $\theta \psi^{-1} \omega^{2}$, multiplied by an elementary extra factor. (Here and in the rest of the introduction, we exclude the special case where $\theta=\omega^{-2}$ and $\psi$ is the trivial character, in which case all the congruence modules vanish.)

We remark that the assumptions above assure us that there is no congruence between $\mathcal{E}(\theta, \psi)$ and other $\Lambda$-adic Eisenstein series, and hence the localization allows us to isolate $\mathcal{E}(\theta, \psi)$. To 
treat more general cases, it seems better to work with the language of components in the sense of Mazur and Wiles [11]; but we do not do this in this paper which is already somewhat lengthy.

One of the motivation of this work was our desire to understand the "Eisenstein components" of the above $\Lambda$-adic objects better. We were also directly motivated by a conjecture (resp. a problem) of Hida (resp. Greenberg and Stevens). Namely, in [4], Hida conjectured that the congruence module in the case (II) above should be expressed in terms of Kubota-Leopoldt $p$-adic $L$-function. On the other hand, in [1], Greenberg and Stevens posed a problem of analyzing the structure of the congruence module in the case (IV). Our result gives affirmative answers to these problems for the local components described above.

Our strategy and the content of this paper is as follows. Section 1 is preliminary: In Section 1.1, we state some general facts about congruence modules, modelled by Hida's account [5], Section 1. In Section 1.2, we introduce $\Lambda$-adic objects we are going to consider. We then give a Poincaré type duality between the group of ordinary $p$-adic modular symbols and the ordinary generalized $p$-adic Eichler-Shimura cohomology group in Section 1.3, and study $\Lambda$-adic Eisenstein series in Section 1.4. In Section 1.5, we state our main theorem (1.5.5).

Now among the four cases (I)-(IV), it may be apparent from our discussions in the classical cases (i)-(iv) that the easiest is the case (I). Indeed, when $\psi=\mathbf{1}$, the trivial character, we applied the same argument as in the classical case to determine the congruence module, in the course of the proof of [15], (5.2.14) (under some restrictive assumptions). It was based on the fact that the "constant term" of $\mathcal{E}(\theta, \psi)$ is given by the $p$-adic $L$-function. However, when $\psi \neq \mathbf{1}$, the constant term of $\mathcal{E}(\theta, \psi)$ vanishes. The classical theory of Eisenstein series then suggests that we should look for the "constant term hidden at some cusp". This is the content of Section 2.

To do this, we make use of a homomorphism of the space of ordinary $\Lambda$-adic modular forms onto the ordinary ( $\Lambda$-adic) cuspidal group whose kernel consists of ordinary $\Lambda$-adic cusp forms. Such a mapping, called the residue mapping, was in fact constructed in our previous work [15] (and complemented by [16]). This will be recalled in Section 2.4. Before this, in Sections 2.1-2.3, we give a detailed study of the ordinary cuspidal group, especially the kernel of the Eisenstein ideal in it. Under the assumption $p \nmid \varphi(N)$, we give an explicit basis of this kernel, which is a free module of rank one over the Iwasawa algebra. The main result of Section 2 is Theorem (2.4.10), which describes the image of $\mathcal{E}(\theta, \psi)$ under the residue mapping in terms of that basis. Its proof, largely computational, is carried out in Sections 2.5-2.6. Via the specializations to classical forms, this ultimately reduces to the computation of the constant terms of $q$-expansions of classical Eisenstein series (of weight 2) at various cusps.

In Section 3, we give the proof of our main theorem. We first settle the case (I) in Section 3.1. The method is almost identical with the classical case (i) above, replacing the "constant term mapping" by our residue mapping, after the key result (2.4.10). The proof for the case (II) is divided into two parts, both dependent on the result in the case (I): We treat the cases where (a) $\psi \neq \mathbf{1}$; and (b) $\theta \psi^{-1} \neq \omega^{-2}$, separately. The proof in the case (a), which is given in Section 3.2, is completely elementary, and in fact the conclusion is derived from the case (I) via simple duality argument (cf. (1.1.12) and (3.2.7) in the text). On the other hand, the proof in the case (b) is far from being elementary. It makes use of the Iwasawa main conjecture for $\mathbf{Q}$ (a theorem of Mazur and Wiles [11], for which we have given a simple proof in our previous work [16]). However, the method itself is not new: It is an adaptation of the one initiated by Harder and Pink [2] and Kurihara [9] to a slightly wider $\Lambda$-adic situation than in [15], 5.3, where we have already settled the case (II) when $\psi=\mathbf{1}$ under additional assumptions. We thus place this argument in Appendix A.

The key ingredient of the proof for the case (III) is the $p$-adic Eichler-Shimura isomorphisms established in our previous works. Such isomorphisms, together with some results on Galois representations on $p$-adic Eichler-Shimura cohomology groups (3.4), easily reduce the problem 
to the case (I). Finally, via the duality obtained in Section 1.3, and the duality between certain space of ordinary $\Lambda$-adic modular forms and its Hecke algebra (3.3.1), the case (IV) is reduced to the case (II). These are given in Section 3.5.

In the appendix, we describe the above mentioned application of the method of Harder-Pink and Kurihara, extending the consideration in [15] to general $(\theta, \psi)$ satisfying (b). We thus look at the Galois representation on the local component, attached to $\mathcal{E}(\theta, \psi)$, of the $p$-adic EichlerShimura cohomology group, and construct a pro- $p$ abelian extension $L$ over the cyclotomic $\mathbf{Z}_{p}$-extension $F_{\infty}$ of some abelian number field. A new feature here is that this pro- $p$ extension may ramify at primes dividing $N$. We analyze such ramifications, and show that the characteristic power series of $\operatorname{Gal}\left(L / F_{\infty}\right)$ coincides with (a twist of) the power series appearing in our main theorem (cf. Theorem (A.2.2)). It is at this stage we need the Iwasawa main conjecture for $\mathbf{Q}$. As a consequence, we find that the ramifications precisely correspond to the "extra factor" in our description of the congruence modules, and that the extension $L$ contains the maximal unramified pro- $p$ abelian extension of $F_{\infty}$ satisfying certain Galois property. This also allows us to settle the case (b) of (II).

\section{Congruence modules and our main result}

\subsection{Congruence modules}

We first give general facts about congruence modules associated with the following situation: Let $R$ be an integral domain with its quotient field $L$. Suppose we are given an exact sequence of flat $R$-modules:

$$
0 \rightarrow A \stackrel{i}{\rightarrow} B \stackrel{\pi}{\rightarrow} C \rightarrow 0 \quad(\text { exact })
$$

together with its splitting over $L$ :

$$
0 \leftarrow A \otimes_{R} L \stackrel{t}{\leftarrow} B \otimes_{R} L \stackrel{s}{\leftarrow} C \otimes_{R} L \leftarrow 0 \quad \text { (exact) }
$$

so that

$$
t \circ\left(i \otimes_{R} 1_{L}\right)=1_{A \otimes_{R} L} \quad \text { and } \quad\left(\pi \otimes_{R} 1_{L}\right) \circ s=1_{C \otimes_{R} L} .
$$

We then clearly have $t(B) \supseteq A$, and the mapping $t \oplus \pi: B \rightarrow t(B) \oplus C$ is injective. Identify $B$ with its image in $t(B) \oplus C$.

LEMMA (1.1.4). - The notation being as above, we have the following canonical isomorphisms of R-modules:

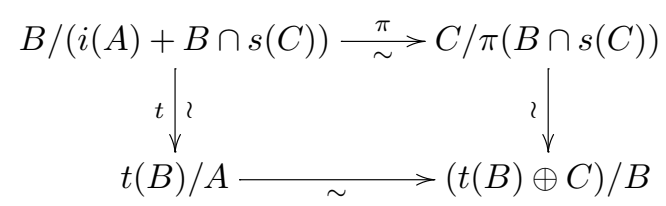

Here, the right vertical (resp. the lower horizontal) arrow is induced from the inclusion of $C$ (resp. $t(B)$ ) into the direct sum $t(B) \oplus C$.

The proof is direct, and left to the reader. We only note here that

$$
B \cap s\left(C \otimes_{R} L\right)=B \cap s(C) .
$$


DEFINITION (1.1.6). - We identify the modules in (1.1.4), and call it the congruence module (or the module of congruences) attached to the exact sequence (1.1.1) with the given splitting (1.1.2). Denote this module by $\mathcal{C}$ in the following.

When $H$ is an $R$-algebra, and (1.1.1) and (1.1.2) are exact sequences of $H$-modules, it is clear that $\mathcal{C}$ is equipped with the obvious structure of an $H$-module.

In the following, for later use, we list some elementary properties of $\mathcal{C}$.

First, we note that the formation of congruence modules commutes with arbitrary flat extensions of integral domains $R \rightarrow R^{\prime}$ : Let $\mathcal{C}^{\prime}$ be the congruence module attached to the base extension of (1.1.1) to $R^{\prime}$ with the obvious splitting over the quotient field of $R^{\prime}$. Then it is easy to see that we have a canonical isomorphism:

$$
\mathcal{C}^{\prime} \cong \mathcal{C} \otimes_{R} R^{\prime}
$$

In the rest of this subsection, we assume that:

$$
\left\{\begin{array}{l}
R \text { is noetherian and factorial; } \\
A, B \text { and } C \text { are finitely generated free } R \text {-modules; } \\
C \text { is a free } R \text {-module of rank one. }
\end{array}\right.
$$

LEMMA (1.1.9). - If we assume the above conditions, then $\mathcal{C}$ is isomorphic to $R /$ a $R$ with a non-zero element $a \in R$.

Proof (cf. Hida [7], Theorem 4.3). - This is easy using (1.1.5): Identifying $C$ with $R, B \cap s(L)$ is principal in $s(R) \cong R$, since it is reflexive.

Next, let us indicate by the superscript " $\vee$ " the $R$-dual: $M^{\vee}:=\operatorname{Hom}_{R}(M, R)$ for an $R$-module $M$. From our assumption (1.1.8), we have the exact sequence dual to (1.1.1):

$$
0 \rightarrow C^{\vee} \stackrel{\pi^{\vee}}{\rightarrow} B^{\vee} \stackrel{i^{\vee}}{\rightarrow} A^{\vee} \rightarrow 0
$$

together with the splitting dual to (1.1.2):

$$
0 \leftarrow C^{\vee} \otimes_{R} L \stackrel{s^{\vee}}{\leftarrow} B^{\vee} \otimes_{R} L \stackrel{t^{\vee}}{\leftarrow} A^{\vee} \otimes_{R} L \leftarrow 0 .
$$

We denote by $\mathcal{C}^{\vee}$ the congruence module associated with this situation.

LEMMA (1.1.12). - Assume (1.1.8) and moreover that $\mathcal{C}^{\vee}$ is generated by one element as an $R$-module. Then $\mathcal{C}^{\vee}$ is isomorphic to $\mathcal{C}$. form:

Proof. - We may assume that $B=R^{n}$ and $C=R$. Then the mapping $\pi$ (resp. $s$ ) is of the

$$
\left[\begin{array}{c}
x_{1} \\
\vdots \\
x_{n}
\end{array}\right] \mapsto \sum_{i=1}^{n} p_{i} x_{i} \text { with } p_{i} \in R \quad\left(\text { resp. } x \mapsto x\left[\begin{array}{c}
c_{1} \\
\vdots \\
c_{n}
\end{array}\right] \text { with } c_{i} \in L\right) .
$$

We have $\sum_{i=1}^{n} p_{i} c_{i}=1$ since $\left(\pi \otimes_{R} 1_{L}\right) \circ s=1_{C \otimes_{R} L}$.

Now we have

$$
\pi(B \cap s(C))=\left\{x \in R \mid x\left[\begin{array}{c}
c_{1} \\
\vdots \\
c_{n}
\end{array}\right] \in R^{n}\right\}
$$


so that $\mathcal{C} \cong R /\left(R:\left(c_{1}, \ldots, c_{n}\right)\right)$, where $\left(c_{1}, \ldots, c_{n}\right)$ is the $R$-submodule of $L$ generated by $c_{1}, \ldots, c_{n}$ and $R:\left(c_{1}, \ldots, c_{n}\right):=\left\{x \in R \mid x\left(c_{1}, \ldots, c_{n}\right) \subseteq R\right\}$. By (1.1.9), we know that $R:\left(c_{1}, \ldots, c_{n}\right)=a R$ with $a \in R$.

On the other hand, identifying $B^{\vee}$ and $C^{\vee}$ with $R^{n}$ and $R$ in a natural manner, we see that $s^{\vee}$ is given by

$$
\left[\begin{array}{c}
x_{1} \\
\vdots \\
x_{n}
\end{array}\right] \mapsto \sum_{i=1}^{n} c_{i} x_{i} .
$$

Consequently, we have: $\mathcal{C}^{\vee} \cong s^{\vee}\left(B^{\vee}\right) / C^{\vee} \cong\left(c_{1}, \ldots, c_{n}\right) / R$.

By our assumption, there is an $\alpha \in L$ such that $\left(c_{1}, \ldots, c_{n}\right)=\alpha R+R$. If $\alpha=b / a^{\prime}$ with coprime $a^{\prime}, b \in R$, then $R:\left(c_{1}, \ldots, c_{n}\right)=a^{\prime} R$, and hence we may assume that $a^{\prime}=a$. We conclude that

$$
\mathcal{C}^{\vee} \cong\left(\frac{b}{a} R+R\right) / R \cong b R / a b R \cong R / a R
$$

\subsection{Basic cohomology groups, Hecke algebras and $\Lambda$-adic modular forms}

We henceforth fix a prime number $p \geqslant 5$. We also fix a complete subfield $K$ of $\mathbf{C}_{p}:=\widehat{\overline{\mathbf{Q}}}_{p}$, and denote by $\mathfrak{o}$ its ring of integers.

Terminology (1.2.1). - For a positive integer $r$, we write $U_{r}$ for the multiplicative subgroup $1+p^{r} \mathbf{Z}_{p}$ of $\mathbf{Z}_{p}^{\times}$. We denote by

$$
\Lambda_{\mathfrak{o}}:=\mathfrak{o} \llbracket 1+p \mathbf{Z}_{p} \rrbracket=\mathfrak{o} \llbracket U_{1} \rrbracket
$$

the Iwasawa algebra over $\mathfrak{o}$. As usual, we fix a topological generator $u_{0}$ of $U_{1}$ and identify $\Lambda_{\mathfrak{o}}$ with the formal power series ring $\mathfrak{o} \llbracket T \rrbracket$ by $u_{0} \leftrightarrow 1+T$.

In the following, we fix our basic notation: For a positive integer $M$, we let $X_{1}(M)$ be the canonical model over $\mathbf{Q}$ of the (complete) modular curve associated with the group $\Gamma_{1}(M)$, whose cusp at infinity is $\mathbf{Q}$-rational. Let $Y_{1}(M)$ be its open subscheme consisting of non-cuspidal points.

Definition (1.2.2). - We fix a positive integer $N$ prime to $p$, and set:

$$
\left\{\begin{array}{l}
E S_{p}(N)_{\mathfrak{o}}:=\left(\lim _{r \geqslant 1} H^{1}\left(X_{1}\left(N p^{r}\right) \otimes_{\mathbf{Q}} \overline{\mathbf{Q}}, \mathbf{Z}_{p}\right)\right) \widehat{\otimes}_{\mathbf{z}_{p}} \mathfrak{o}, \\
G E S_{p}(N)_{\mathfrak{o}}:=\left(\varliminf_{r \geqslant 1}^{\lim } H^{1}\left(Y_{1}\left(N p^{r}\right) \otimes_{\mathbf{Q}} \overline{\mathbf{Q}}, \mathbf{Z}_{p}\right)\right) \widehat{\otimes}_{\mathbf{z}_{p}} \mathfrak{o}, \\
M S_{p}(N)_{\mathfrak{o}}:=\left(\lim _{r \geqslant 1} H_{c}^{1}\left(Y_{1}\left(N p^{r}\right) \otimes_{\mathbf{Q}} \overline{\mathbf{Q}}, \mathbf{Z}_{p}\right)\right) \widehat{\otimes}_{\mathbf{z}_{p}} \mathfrak{o} .
\end{array}\right.
$$

Here, all the cohomology groups in the right-hand side are étale cohomology groups, and $\widehat{\otimes}$ mean the completed tensor products viewing the projective limits in the parentheses as pro- $p$ abelian groups.

The Galois group $\operatorname{Gal}(\overline{\mathbf{Q}} / \mathbf{Q})$ acts on these groups o-linearly in a natural manner. On the other hand, Hecke correspondences on $X_{1}\left(N p^{r}\right)$ or $Y_{1}\left(N p^{r}\right)$ give rise to endomorphisms of individual first cohomology groups in the right-hand side. Precisely, in this paper, we will always 
consider the covariant action (except in the proof of (1.3.3) below), and denote by $T^{*}(n)$ and $T^{*}(q, q)$ the endomorphisms attached to the usual Hecke correspondences (cf. e.g. [13], 7.3, 7.4). $T^{*}(n)$ and $T^{*}(q, q)$ on the first cohomology groups commute with the trace mappings, and hence define endomorphisms of the groups in the left-hand side, which we denote by the same symbols. Especially, we have Hida's idempotent

$$
e^{*}:=\lim _{n \rightarrow \infty} T^{*}(p)^{n !}
$$

acting on the groups above.

The groups $E S_{p}(N)_{\mathfrak{o}}, G E S_{p}(N)_{\mathfrak{o}}$ and $M S_{p}(N)_{\mathfrak{o}}$ are then naturally equipped with the structure of modules over

$$
\mathfrak{o}\left[\left[\varliminf_{r \geqslant 1}^{\lim }\left(\mathbf{Z} / N p^{r} \mathbf{Z}\right)^{\times}\right]\right]=\mathfrak{o}\left[(\mathbf{Z} / N p \mathbf{Z})^{\times}\right] \llbracket U_{1} \rrbracket
$$

in such a way that (the image of) a positive integer $q$ prime to $N p$ in $\varliminf_{r \geqslant 1}\left(\mathbf{Z} / N p^{r} \mathbf{Z}\right)^{\times}$acts as $T^{*}(q, q)$. In particular, we can consider them as modules over the Iwasawa algebra $\Lambda_{\mathfrak{o}}$. One of the fundamental fact discovered by Hida ([6], Theorem 3.1, complemented by [15], (1.3.5)) is that the ordinary parts $e^{*} E S_{p}(N)_{\mathfrak{o}}, e^{*} G E S_{p}(N)_{\mathfrak{o}}$ and $e^{*} M S_{p}(N)_{\mathfrak{o}}$ are all free of finite rank over $\Lambda_{\mathfrak{o}}$.

We also know that $e^{*} E S_{p}(N)_{\mathfrak{o}}$ (resp. $\left.e^{*} G E S_{p}(N)_{\mathfrak{o}}\right)$ are modules over Hida's universal ordinary $p$-adic Hecke algebras:

$$
\left\{\begin{array}{l}
e^{*} h^{*}(N ; \mathfrak{o}) \hookrightarrow \operatorname{End}\left(e^{*} E S_{p}(N)_{\mathfrak{o}}\right), \\
e^{*} \mathcal{H}^{*}(N ; \mathfrak{o}) \hookrightarrow \operatorname{End}\left(e^{*} G E S_{p}(N)_{\mathfrak{o}}\right),
\end{array}\right.
$$

which are generated by all $T^{*}(n)$ and $T^{*}(q, q)$ over $\Lambda_{\mathfrak{o}}$. We can consider $e^{*} h^{*}(N ; \mathfrak{o})$ and $e^{*} \mathcal{H}^{*}(N ; \mathfrak{o})$ as algebras over $\mathfrak{o}\left[(\mathbf{Z} / N p \mathbf{Z})^{\times}\right] \llbracket U_{1} \rrbracket$ in the same manner as above; and again by Hida ([4], Theorem 3.1; cf. also [15], (1.5.7)), these are finite and flat over $\Lambda_{\mathfrak{o}}$.

On the other hand, there are notions of ordinary $\Lambda_{\mathfrak{o}}$-adic cusp forms and $\Lambda_{\mathfrak{o}}$-adic modular forms, introduced by Hida [4] and Wiles [21]. We use the same notation and convention as in [14], Section 2 and [15], Section 2, and denote by $e S\left(N ; \Lambda_{\mathfrak{o}}\right)$ and $e M\left(N ; \Lambda_{\mathfrak{o}}\right)$ the spaces of such forms, respectively. We only recall here that they consist of formal power series $\mathcal{F}=\sum_{n=0}^{\infty} a_{n}(T) q^{n} \in \Lambda_{\mathfrak{o}} \llbracket q \rrbracket$ such that their specializations

$$
\mathcal{F}_{\varepsilon, d}:=\sum_{n=0}^{\infty} a_{n}\left(\varepsilon\left(u_{0}\right) u_{0}^{d}-1\right) \cdot q^{n}
$$

are ordinary cusp forms (resp. modular forms), with coefficients in the ring generater by the values of $\varepsilon$ over $\mathfrak{o}$, of wight $d+2$ with prescribed level and the $p$-part of the Nebentypus character. Here, $\varepsilon$ is any $\overline{\mathbf{Q}}^{\times}$-valued character of finite order of $U_{1}$, and $d$ is any non-negative integer. $e S\left(N ; \Lambda_{\mathfrak{o}}\right)$ and $e M\left(N ; \Lambda_{\mathfrak{o}}\right)$ are $\Lambda_{\mathfrak{o}}$-modules in an obvious way; and are in fact finite and free over $\Lambda_{\mathfrak{o}}$. One can define the Hecke operators $T(n)$ and $T(q, q)$ on these spaces in such a way that they correspond to the usual ones via the specializations (1.2.5) to classical forms. The Hecke algebras generated by all $T(n)$ and $T(q, q)$ over $\Lambda_{\mathfrak{o}}$

$$
\left\{\begin{array}{l}
e h(N ; \mathfrak{o}) \hookrightarrow \operatorname{End}\left(e S\left(N ; \Lambda_{\mathfrak{o}}\right)\right), \\
e \mathcal{H}(N ; \mathfrak{o}) \hookrightarrow \operatorname{End}\left(e M\left(N ; \Lambda_{\mathfrak{o}}\right)\right),
\end{array}\right.
$$


are canonically $\Lambda_{\mathfrak{o}}$-isomorphic to $e^{*} h^{*}(N ; \mathfrak{o})$ and $e^{*} \mathcal{H}^{*}(N ; \mathfrak{o})$, respectively, via the correspondence: $T(n) \leftrightarrow T^{*}(n)$ and $T(q, q) \leftrightarrow T^{*}(q, q)$.

\subsection{Duality between $e^{*} G E S_{p}(N)_{\mathfrak{o}}$ and $e^{*} M S_{p}(N)_{\mathrm{o}}$}

Let us denote by

$$
\iota: \lim _{r \geqslant 1}\left(\mathbf{Z} / N p^{r} \mathbf{Z}\right)^{\times} \hookrightarrow \mathfrak{o}\left[[\underbrace{\lim }_{r \geqslant 1}\left(\mathbf{Z} / N p^{r} \mathbf{Z}\right)^{\times}]\right]
$$

the natural inclusion, to distinguish the image of an element in the left-hand side in the completed group algebra from a scalar. Also in what follows, let

$$
\kappa: \operatorname{Gal}(\overline{\mathbf{Q}} / \mathbf{Q}) \rightarrow \mathbf{Z}_{p}^{\times}
$$

be the $p$-cyclotomic character.

The main result of this subsection is the following

THEOREM (1.3.3). - There is a canonical $\Lambda_{\mathfrak{o}}$-bilinear form

$$
\{,\}: e^{*} M S_{p}(N)_{\mathfrak{o}} \times e^{*} G E S_{p}(N)_{\mathfrak{o}} \rightarrow \Lambda_{\mathfrak{o}}
$$

which enjoys the following properties:

(i) It gives a perfect duality of free $\Lambda_{\mathfrak{o}}$-modules.

(ii) It commutes with the Hecke operators: For any $x \in e^{*} M S_{p}(N)_{\mathfrak{o}}$ and $y \in e^{*} G E S_{p}(N)_{\mathfrak{o}}$, we have

$$
\left\{\begin{array}{l}
\left\{x \mid T^{*}(n), y\right\}=\left\{x, y \mid T^{*}(n)\right\}, \\
\left\{x \mid T^{*}(q, q), y\right\}=\left\{x, y \mid T^{*}(q, q)\right\} .
\end{array}\right.
$$

(iii) It has the following compatibility with the Galois action: For $\sigma \in \operatorname{Gal}(\overline{\mathbf{Q}} / \mathbf{Q})$, let $a(\sigma)$ be the unique element of $\lim _{r \geqslant 1}\left(\mathbf{Z} / N p^{r} \mathbf{Z}\right)^{\times}$such that $\zeta^{\sigma}=\zeta^{a(\sigma)}$ for all $N p^{r}$ th roots of unity $(r \geqslant 1)$. Then we have:

$$
\left\{x^{\sigma}, y^{\sigma}\right\}=\kappa(\sigma)^{-1}\left\{x, \iota\left(a(\sigma)^{-1}\right) \cdot y\right\}
$$

We studied similar $\Lambda_{\mathfrak{o}}$-bilinear form on $e^{*} E S_{p}(N)_{\mathfrak{o}} \times e^{*} E S_{p}(N)_{\mathfrak{o}}$ in [14], Section 4; and the proof in the present case is almost identical. So, we only sketch the method referring to [14], 4.1 and 4.2 for details.

First note that we may assume that $\mathfrak{o}=\mathbf{Z}_{p}$, since then the general case follows by tensoring $\Lambda_{\mathfrak{o}}$ over $\Lambda_{\mathbf{Z}_{p}}$. Now for each integer $r \geqslant 1$, we write $Y_{r}:=Y_{1}\left(N p^{r}\right)$ and indicate by bar the base extension from $\mathbf{Q}$ to $\overline{\mathbf{Q}}$ for notational simplicity.

For each $r \geqslant 1$, we recall that there is a $p$-adic sheaf $F_{\mathcal{M}_{r}}=F_{\mathbf{Z}_{p} \llbracket Z_{r} \rrbracket}$ on the étale site $Y_{r \text {,ét }}$ of $Y_{r}$, and $G E S_{p}(N) \mathbf{z}_{p}$ (resp. $\left.M S_{p}(N) \mathbf{z}_{p}\right)$ is canonically isomorphic to $H^{1}\left(\bar{Y}_{r}, F_{\mathcal{M}_{r}}\right.$ ) (resp. $H_{c}^{1}\left(\bar{Y}_{r}, F_{\mathcal{M}_{r}}\right)$ ) ([14], 1.2). On the other hand, there is another $p$-adic sheaf $F_{\mathcal{M}_{r}^{\prime}}$ and a pairing of sheaves:

$$
F_{\mathcal{M}_{r}} \times F_{\mathcal{M}_{r}^{\prime}} \rightarrow F_{\mathbf{Z}_{p} \llbracket U_{r} \rrbracket}
$$

on $\bar{Y}_{r, \text { ét }}([14], 4.1)$, where $F_{\mathbf{Z}_{p} \llbracket U_{r} \rrbracket}$ is the constant sheaf corresponding to

$$
\mathbf{Z}_{p} \llbracket U_{r} \rrbracket=\mathbf{Z}_{p} \llbracket 1+p^{r} \mathbf{Z}_{p} \rrbracket .
$$


This then gives rise to the cup product pairing:

$$
{ }_{s} \cup_{r}: H_{c}^{1}\left(\bar{Y}_{s}, F_{\mathcal{M}_{r}}\right) \times H^{1}\left(\bar{Y}_{s}, F_{\mathcal{M}_{r}^{\prime}}\right) \rightarrow H_{c}^{2}\left(\bar{Y}_{s}, F_{\mathbf{Z}_{p} \llbracket U_{r} \rrbracket}\right) \cong \mathbf{Z}_{p} \llbracket U_{r} \rrbracket(-1)
$$

for integers $s \geqslant r \geqslant 1$. The inclusions of modules

$$
\left\{\begin{array}{l}
i_{r}^{s}: \mathcal{M}_{s} \hookrightarrow \mathcal{M}_{r} \\
i_{r}^{\prime s}: \mathcal{M}_{s}^{\prime} \hookrightarrow \mathcal{M}_{r}^{\prime}
\end{array}\right.
$$

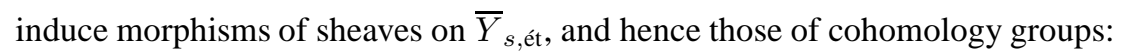

$$
\left\{\begin{array}{l}
i_{r *}^{s}: H_{c}^{1}\left(\bar{Y}_{s}, F_{\mathcal{M}_{s}}\right) \rightarrow H_{c}^{1}\left(\bar{Y}_{s}, F_{\mathcal{M}_{r}}\right) \\
i_{r *}^{\prime s}: H^{1}\left(\bar{Y}_{s}, F_{\mathcal{M}_{s}^{\prime}}\right) \rightarrow H^{1}\left(\bar{Y}_{s}, F_{\mathcal{M}_{s}^{\prime}}\right)
\end{array}\right.
$$

Using the operator " $\tau_{r}$ " (resp. " $\sigma_{\alpha}^{-1 ")}$ given by the same formula as [14], (4.1.3) (resp. (4.1.13)) on $H^{1}\left(\bar{Y}_{r}, F_{\mathcal{M}_{r}}\right) \cong H^{1}\left(\Gamma_{1}\left(N p^{r}\right), \mathcal{M}_{r}\right)$ (the group cohomology) etc., we define

$$
\{,\}_{r}: H_{c}^{1}\left(\bar{Y}_{r}, F_{\mathcal{M}_{r}}\right) \times H^{1}\left(\bar{Y}_{r}, F_{\mathcal{M}_{r}}\right) \rightarrow \Lambda_{\mathbf{Z}_{p}}
$$

by the formula

$$
\{x, y\}_{r}:=\sum_{\alpha \in U_{1} / U_{r}} i_{1 *}^{r}(x)_{r} \cup_{1}\left(i_{1 *}^{\prime r}\left(y\left|T^{*}(p)^{r}\right| \tau_{r}\right) \mid \sigma_{\alpha}^{-1}\right)
$$

fixing an isomorphism $\mathbf{Z}_{p}(-1) \cong \mathbf{Z}_{p}$. With these definitions, it is an easy matter to obtain the same result as in [14], 4.1 and 4.2. Especially, the pairings $\{,\}_{r}$ are compatible with the canonical isomorphisms $G E S_{p}(N) \mathbf{z}_{p} \cong H^{1}\left(\bar{Y}_{r}, F_{\mathcal{M}_{r}}\right)$ and $M S_{p}(N) \mathbf{z}_{p} \cong H_{c}^{1}\left(\bar{Y}_{r}, F_{\mathcal{M}_{r}}\right)$ when $r \geqslant 1$ varies, and define the pairing on the whole spaces

$$
\{,\}: M S_{p}(N) \mathbf{z}_{p} \times G E S_{p}(N) \mathbf{z}_{p} \rightarrow \Lambda_{\mathbf{z}_{p}}
$$

which specializes to certain ("twisted") cup product pairing

$$
H_{c}^{1}\left(\bar{Y}_{r}, F_{S^{d}\left(\mathbf{z}_{p}\right)}\right) \times H^{1}\left(\bar{Y}_{r}, F_{S^{d}\left(\mathbf{z}_{p}\right)}\right) \rightarrow \Lambda_{\mathbf{z}_{p}} /\left(\omega_{r, d}\right)
$$

for each $r \geqslant 1$ and $d \geqslant 0$, as in [14], (4.2.5) (cf. also [15], (1.4.2)). This $\{$,$\} already satisfies the$ compatibilities (ii) and (iii) of the theorem (use [15], (3.3.4) and (4.2.4) for (iii)), and induces the pairing in the theorem on the ordinary parts.

Finally, we show the assertion (i) of the theorem. Since $e^{*} G E S_{p}(N) \mathbf{z}_{p}$ and $e^{*} M S_{p}(N) \mathbf{z}_{p}$ are finite and free over $\Lambda_{\mathbf{Z}_{p}}$, it is sufficient to show that the pairing reduced modulo $(T)$ is perfect. By the above mentioned compatibility with specializations, this is reduced to the perfectness of the pairing between free $\mathbf{Z}_{p}$-modules:

$$
e^{*} H_{c}^{1}\left(\bar{Y}_{1}, \mathbf{Z}_{p}\right) \times e^{*} H^{1}\left(\bar{Y}_{1}, \mathbf{Z}_{p}\right) \rightarrow \mathbf{Z}_{p}\left(\cong \mathbf{Z}_{p}(-1)\right)
$$

defined by $(x, y) \mapsto x \cup\left(y\left|T^{*}(p)\right| \tau_{1}\right)$. However, $y \mapsto y\left|T^{*}(p)\right| \tau_{1}$ gives an isomorphism of $e^{*} H^{1}\left(\bar{Y}_{1}, \mathbf{Z}_{p}\right)$ onto the ordinary part $e H^{1}\left(\bar{Y}_{1}, \mathbf{Z}_{p}\right)$ with respect to $T(p)$, which is adjoint to $T^{*}(p)$ with respect to the cup product. Our claim therefore follows from the usual Poincare duality theorem. 
For the same reason, the pairing

$$
\{,\}: e^{*} E S_{p}(N)_{\mathfrak{o}} \times e^{*} E S_{p}(N)_{\mathfrak{o}} \rightarrow \Lambda_{\mathfrak{o}}
$$

studied in [14] is also perfect.

There is a natural injection $i: e^{*} E S_{p}(N)_{\mathfrak{o}} \rightarrow e^{*} G E S_{p}(N)_{\mathfrak{o}}$ and a natural surjection

$$
\pi: e^{*} M S_{p}(N)_{\mathfrak{o}} \rightarrow e^{*} E S_{p}(N)_{\mathfrak{o}} .
$$

It follows easily from the description of $\{$,$\} above that$

$$
\{\pi(x), y\}=\{x, i(y)\}
$$

for all $x \in e^{*} M S_{p}(N)_{\mathfrak{o}}$ and $y \in e^{*} E S_{p}(N)_{\mathfrak{o}}$.

Note that, by the theorem above, the Hecke algebra defined similarly as (1.2.4) for $e^{*} M S_{p}(N)_{\mathfrak{o}}$ is canonically isomorphic to $e^{*} \mathcal{H}^{*}(N ; \mathfrak{o})$; and that this makes $e^{*} M S_{p}(N)_{\mathfrak{o}}$ into a faithful $e^{*} \mathcal{H}^{*}(N ; \mathfrak{o})$-module.

\section{4. $\Lambda$-adic Eisenstein series}

In general, let $\omega$ denote the Teichmüller character, and set

$$
\langle a\rangle:=a \omega(a)^{-1} \in U_{1} \quad \text { for } a \in \mathbf{Z}_{p}^{\times} .
$$

Let $N$ be as in previous subsections, and consider Dirichlet characters $\theta$ and $\psi$ defined modulo $u$ and $v$, respectively. (Dirichlet characters will be assumed to be primitive unless otherwise stated.) We will always assume that $\theta \psi(-1)=1, v$ is prime to $p$, and $u v$ is a divisor of $N p$.

Fix a finite extension $k$ of $\mathbf{Q}_{p}$ which contains all the values of $\theta$ and $\psi$. We denote by $\mathfrak{r}$ its ring of integers, and take a prime element $\varpi$ of $\mathfrak{r}$. The $\Lambda$-adic Eisenstein series to be considered is of the following form (cf. [15], 2.3):

$$
\mathcal{E}(\theta, \psi ; c):=\delta(\psi) G\left(T, \theta \omega^{2}\right)+\sum_{n=1}^{\infty}\left(\sum_{\substack{0<t \mid n \\ p \nmid t}} \theta(t) \psi\left(\frac{n}{t}\right) A_{t}(T)\right) q^{c n} .
$$

Here, $c$ is a positive divisor of $N p / u v$ prime to $p$,

$$
\begin{aligned}
& \delta(\psi):= \begin{cases}\frac{1}{2} & \text { if } \psi=\mathbf{1}:=(\text { the trivial character }), \\
0 & \text { otherwise, }\end{cases} \\
& A_{t}(T):=t(1+T)^{s(t)}=t \iota(\langle t\rangle) \text { if }\langle t\rangle=u_{0}^{s(t)}
\end{aligned}
$$

and $G\left(T, \theta \omega^{2}\right)$ is a twist of the Kubota-Leopoldt $p$-adic $L$-function in the following sense: It belongs to $\Lambda_{\mathfrak{r}}$ unless $\theta \omega^{2}=\mathbf{1}$, while $\left((1+T)-u_{0}^{-2}\right) G(T, \mathbf{1}) \in \Lambda_{\mathbf{Z}_{p}}$, and satisfies

$$
G\left(\varepsilon\left(u_{0}\right) u_{0}^{s}-1, \theta \omega^{2}\right)=L_{p}\left(-1-s, \theta \omega^{2} \varepsilon\right)
$$

for every character $\varepsilon$ of finite order of $U_{1}$ (which we identify with a Dirichlet character of the second kind), $L_{p}$ being the $p$-adic $L$-function. We henceforth set

$$
\mathcal{E}(\theta, \psi):=\mathcal{E}(\theta, \psi ; 1) .
$$


$\mathcal{E}(\theta, \psi ; c)$ then belongs to $e M\left(N ; \Lambda_{\mathfrak{r}}\right)$ unless $\theta=\omega^{-2}$ and $\psi=\mathbf{1}$; while $\left((1+T)-u_{0}^{-2}\right) \times$ $\mathcal{E}\left(\omega^{-2}, \mathbf{1} ; c\right)$ belongs to $\operatorname{e} M\left(N ; \Lambda_{\mathbf{Z}_{p}}\right)$. The $(\varepsilon, d)$-specialization $\mathcal{E}(\theta, \psi ; c)_{\varepsilon, d}$ in the sense of (1.2.5) is explicitly given by

$$
-\delta(\psi)\left(1-\left(\theta \varepsilon \omega^{-d}\right)(p) p^{d+1}\right) \frac{B_{d+2, \theta \varepsilon \omega^{-d}}}{d+2}+\sum_{n=1}^{\infty}\left(\sum_{\substack{0<t \mid n \\ p \nmid t}}\left(\theta \varepsilon \omega^{-d}\right)(t) \psi\left(\frac{n}{t}\right) t^{d+1}\right) q^{c n} .
$$

When $c=1$, this gives a modular form of weight $d+2$ with respect to the group $\Gamma_{1}\left(u v p^{r} /(u, p)\right)$ if $\operatorname{Ker}(\varepsilon)=U_{r}$. Its Nebentypus character is the (possibly imprimitive) character $\theta \varepsilon \omega^{-d} \psi$ defined modulo $u v p^{r} /(u, p)$, and it is a common eigen function of all $T(n)$ of level $u v p^{r} /(u, p)$. One then obtains the following lemma comparing the specializations:

LEMMA (1.4.8). - When $(\theta, \psi) \neq\left(\omega^{-2}, \mathbf{1}\right)$, we have

$$
\left\{\begin{array}{l}
\mathcal{E}(\theta, \psi ; c) \mid T(q, q)=(\theta \psi)(q) \iota(\langle q\rangle) \mathcal{E}(\theta, \psi ; c) \\
\mathcal{E}(\theta, \psi ; c) \mid T(l)=(\theta(l) l \iota(\langle l\rangle)+\psi(l)) \mathcal{E}(\theta, \psi ; c) \\
\mathcal{E}(\theta, \psi ; c) \mid T(p)=\psi(p) \mathcal{E}(\theta, \psi ; c)
\end{array}\right.
$$

Here, $q$ is any positive integer prime to $N p$, and $l$ is a prime number not dividing $N p$. Also, for $a \in(\mathbf{Z} / N p \mathbf{Z})^{\times}$, the action of $\iota(a)$ on $\mathcal{E}(\theta, \psi ; c)$ is multiplication by $(\theta \psi)(a)$.

When $u v=N$ or $N p$ (and hence $c=1)$, the second formula above for $\mathcal{E}(\theta, \psi) \mid T(l)$ is valid for all prime numbers $l \neq p$.

The same holds for $\left((1+T)-u_{0}^{-2}\right) \mathcal{E}\left(\omega^{-2}, \mathbf{1} ; c\right)$ when $(\theta, \psi)=\left(\omega^{-2}, \mathbf{1}\right)$.

LEMma (1.4.9). - Assume that $p$ does not divide $\varphi(N)$ (the Euler function). Let $\theta_{i}$ and $\psi_{i}$ be Dirichlet characters defined modulo $u_{i}$ and $v_{i}$ satisfying the conditions at the beginning of this subsection; and let $c_{i}$ be positive divisors of $N p / u_{i} v_{i}$ prime to $p(i=1,2)$, respectively. Assume that $\mathfrak{r}$ contains all the values of $\theta_{i}$ and $\psi_{i}$.

(i) The eigenvalues of $T(l)$ for $\mathcal{E}\left(\theta_{1}, \psi_{1} ; c_{1}\right)$ and $\mathcal{E}\left(\theta_{2}, \psi_{2} ; c_{2}\right)$ are congruent modulo $(\varpi, T)$ for all prime numbers $l$ not dividing $N p$, if and only if

$$
\left\{\begin{array}{l}
\theta_{1}=\theta_{2} \text { and } \psi_{1}=\psi_{2} ; \text { or } \\
\theta_{2}=\psi_{1} \omega^{-1} \text { and } \psi_{2}=\theta_{1} \omega .
\end{array}\right.
$$

(ii) Assume moreover that $u_{i} v_{i}=N$ or $N p$ for $i=1,2$. Then the eigenvalues of $T(l)$ for $\mathcal{E}\left(\theta_{1}, \psi_{1}\right)$ and $\mathcal{E}\left(\theta_{2}, \psi_{2}\right)$ are congruent modulo $(\varpi, T)$ for all prime numbers $l$ if and only if

$$
\left\{\begin{array}{l}
\theta_{1}=\theta_{2} \text { and } \psi_{1}=\psi_{2} ; \text { or } \\
\theta_{2}=\psi_{1} \omega^{-1}, \psi_{2}=\theta_{1} \omega \text { and }\left(\theta_{1} \omega \psi_{1}^{-1}\right)(p)=1
\end{array}\right.
$$

Proof. - We first treat the case (i). If we have the congruence for the eigenvalues as stated, then by (1.4.8) above, we have

$$
\theta_{1}(l) l \iota(\langle l\rangle)+\psi_{1}(l) \equiv \theta_{2}(l) l \iota(\langle l\rangle)+\psi_{2}(l) \quad \bmod (\varpi, T)
$$

or equivalently,

$$
\left(\theta_{1} \omega\right)(l)+\psi_{1}(l) \equiv\left(\theta_{2} \omega\right)(l)+\psi_{2}(l) \quad \bmod (\varpi)=\varpi \mathfrak{r}
$$


for all prime numbers $l \nmid N p$. Then by Artin’s lemma on the linear independence of characters, we have either

$$
\left\{\begin{array}{l}
\psi_{1} \equiv \psi_{2} \text { and } \theta_{1} \omega \equiv \theta_{2} \omega \bmod (\varpi) ; \text { or } \\
\psi_{1} \equiv \theta_{2} \omega \text { and } \psi_{2} \equiv \theta_{1} \omega \bmod (\varpi) .
\end{array}\right.
$$

In the first case, our assumption on $N$ implies that $\psi_{1}=\psi_{2}$, and $\theta_{1}=\theta_{2}$. In the second case, we have $\psi_{1}=\theta_{2} \omega$ and $\psi_{2}=\theta_{1} \omega$. Note that, since the conductors of $\psi_{1}$ and $\psi_{2}$ are prime to $p$, we must have $\left.\theta_{i}\right|_{(\mathbf{Z} / p \mathbf{Z})^{\times}}=\omega^{-1}$ in this case. The converse is obvious.

We turn to the case (ii). For the "only if" part, we note that the assumption implies that $\psi_{1}(p)=\psi_{2}(p)$ by (1.4.8). Thus in the case where $\theta_{2}=\psi_{1} \omega^{-1}$ and $\psi_{2}=\theta_{1} \omega$, we must have $\left(\theta_{1} \omega \psi_{1}^{-1}\right)(p)=1$. The "if" part is clear.

Note that $\left(\theta_{1} \omega \psi_{1}^{-1}\right)(p)=1$ if and only if the $p$-adic $L$-function $L_{p}\left(s, \theta_{1} \psi_{1}^{-1} \omega^{2}\right)$ has the socalled trivial zero (or exceptional zero) at $s=0$.

Terminology (1.4.10). - We say that the pair $(\theta, \psi)$ as above is exceptional if $\left.\theta\right|_{(\mathbf{Z} / p \mathbf{Z}) \times}=\omega^{-1}$ and $\left(\theta \omega \psi^{-1}\right)(p)=1$.

\subsection{Main result}

We keep the notation of previous subsections. Especially, we fix $\theta$ and $\psi$ of conductors $u$ and $v$ whose values are contained in $\mathfrak{r}$, as in Section 1.4.

Definition (1.5.1). - Assume that $u v=N$ or $N p$. We define the Eisenstein ideal associated with $(\theta, \psi)$, denoted by $\mathcal{I}(\theta, \psi)$, as the annihilator of $\mathcal{E}(\theta, \psi)$ in $e \mathcal{H}(N ; \mathfrak{r})$. We set

$$
\mathfrak{M}(\theta, \psi):=(\mathcal{I}(\theta, \psi), \varpi, T) .
$$

We denote by $I(\theta, \psi)$ (resp. $\mathfrak{m}(\theta, \psi))$ the image of $\mathcal{I}(\theta, \psi)$ (resp. $\mathfrak{M}(\theta, \psi))$ by the canonical surjection: $e \mathcal{H}(N ; \mathfrak{r}) \rightarrow e h(N ; \mathfrak{r})$.

We also denote by $\mathcal{I}^{*}(\theta, \psi)$ and $\mathfrak{M}^{*}(\theta, \psi)$ (resp, $I^{*}(\theta, \psi)$ and $\mathfrak{m}^{*}(\theta, \psi)$ ) the corresponding ideals of $e^{*} \mathcal{H}^{*}(N ; \mathfrak{r})$ (resp. $e^{*} h^{*}(N ; \mathfrak{r})$ ) via the canonical isomorphism stated at the end of Section 1.2.

It is easy to see that $\mathcal{I}(\theta, \psi)$ is in fact the ideal of $e \mathcal{H}(N ; \mathfrak{r})$ generated by

$$
T(n)-\sum_{\substack{0<t \mid n \\ p \nmid t}} \theta(t) \psi\left(\frac{n}{t}\right) t \iota(\langle t\rangle)
$$

for all $n \geqslant 1$; or by such elements for prime numbers $n$ together with $T(q, q)-(\theta \psi)(q) \iota(\langle q\rangle)$ for all $q$ prime to $N p$. Clearly, $\mathfrak{M}(\theta, \psi)$ is a maximal ideal of $e \mathcal{H}(N ; \mathfrak{r})$; but note that $I(\theta, \psi)$ and $\mathfrak{m}(\theta, \psi)$ may not be proper ideals of $e h(N ; \mathfrak{r})$ in general.

We now make the following assumption:

$$
\left\{\begin{array}{l}
p \text { does not divide } \varphi(N) \\
u v=N \text { or } N p, \text { and }(\theta, \psi) \text { is not exceptional (1.4.10). }
\end{array}\right.
$$

We put

$$
\xi:=\theta \psi^{-1}
$$


When $(\theta, \psi) \neq\left(\omega^{-2}, \mathbf{1}\right)$, we set:

$$
A(T ; \theta, \psi):=\left(\prod_{\substack{l \mid N \\ l \nmid \operatorname{cond}(\xi)}}\left((1+T)^{s(l)}-\left(\xi \omega^{2}\right)^{-1}(l)\langle l\rangle^{-2}\right)\right) G\left(T, \xi \omega^{2}\right)
$$

where the product ranges over all prime numbers $l$ dividing $N$ but not dividing the conductor $\operatorname{cond}(\xi)$ of $\xi . s(l) \in \mathbf{Z}_{p}$ (resp. $G\left(T, \xi \omega^{2}\right)$ ) is defined in (1.4.4) (resp. (1.4.5)). It is easy to see that our assumption $(\theta, \psi) \neq\left(\omega^{-2}, \mathbf{1}\right)$ ensures us that this in fact belongs to $\Lambda_{\mathfrak{r}}$ even if $\xi \omega^{2}=\mathbf{1}$.

Finally, set $\mathfrak{M}:=\mathfrak{M}(\theta, \psi)$ and $\mathfrak{M}^{*}:=\mathfrak{M}^{*}(\theta, \psi)$ for simplicity, and indicate by the subscript " $\mathfrak{M}$ " or " $\mathfrak{M}^{*}$ " the localization at that maximal ideal.

THEOREM (1.5.5). - Assume (1.5.2). We have the following exact sequences of free $\Lambda_{\mathfrak{r}}$-modules:

$$
\left\{\begin{array}{l}
(\mathrm{I}): 0 \rightarrow e S\left(N ; \Lambda_{\mathfrak{r}}\right)_{\mathfrak{M}} \rightarrow e M\left(N ; \Lambda_{\mathfrak{r}}\right)_{\mathfrak{M}} \rightarrow \Lambda_{\mathfrak{r}} \rightarrow 0 ; \\
(\mathrm{II}): 0 \rightarrow \mathcal{I}(\theta, \psi)_{\mathfrak{M}} \rightarrow e \mathcal{H}(N ; \mathfrak{r})_{\mathfrak{M}} \rightarrow \Lambda_{\mathfrak{r}} \rightarrow 0 ; \\
(\mathrm{III}): 0 \rightarrow e^{*} E S_{p}(N)_{\mathfrak{r}, \mathfrak{M}^{*}} \rightarrow e^{*} G E S_{p}(N)_{\mathfrak{r}, \mathfrak{M}^{*}} \rightarrow \Lambda_{\mathfrak{r}} \rightarrow 0 ; \\
(\mathrm{IV}): 0 \rightarrow \Lambda_{\mathfrak{r}} \rightarrow e^{*} M S_{p}(N)_{\mathfrak{r}, \mathfrak{M}^{*}} \rightarrow e^{*} E S_{p}(N)_{\mathfrak{r}, \mathfrak{M}^{*}} \rightarrow 0 .
\end{array}\right.
$$

When tensored with the quotient field of $\Lambda_{\mathfrak{r}}$ over $\Lambda_{\mathfrak{r}}$, (I) and (II) (resp. (III) and (IV)) split uniquely as modules over e $\mathcal{H}(N ; \mathfrak{r})_{\mathfrak{M}}\left(\right.$ resp. $\left.e^{*} \mathcal{H}^{*}(N ; \mathfrak{r})_{\mathfrak{M}^{*}}\right)$. The associated congruence modules (1.1.6) are all isomorphic to

$$
\Lambda_{\mathfrak{r}} /(A(T ; \theta, \psi))
$$

unless $(\theta, \psi)=\left(\omega^{-2}, \mathbf{1}\right)$; while the congruence modules all vanish when $(\theta, \psi)=\left(\omega^{-2}, \mathbf{1}\right)$.

\section{Residues of $\Lambda$-adic Eisenstein series}

\subsection{Cuspidal groups}

We first consider the cuspidal groups over $\mathfrak{o}$ for the curves $X_{1}\left(N p^{r}\right)$ and the Hecke operators on them; and will turn to their projective limit in Section 2.3. We note that such groups already appeared in a work of Kubert and Lang [10] in their study of cuspidal divisor class groups, and also in Mazur and Wiles [11]. We begin by the description of the cusps of $\Gamma_{1}(M)$ following Shimura [18], 1.6.

Now for a positive integer $M$, we set

$$
A_{M}:=\left\{\left[\begin{array}{l}
x \\
y
\end{array}\right] \in(\mathbf{Z} / M \mathbf{Z})^{\oplus 2} \mid(x, y)=1 \text { in } \mathbf{Z} / M \mathbf{Z}\right\} / \sim
$$

where " $\sim$ " is the equivalence relation defined by

$$
\left[\begin{array}{l}
x \\
y
\end{array}\right] \sim\left[\begin{array}{l}
x^{\prime} \\
y^{\prime}
\end{array}\right] \quad \text { if and only if } \quad y=y^{\prime} \text { and } x \equiv x^{\prime} \bmod y(\mathbf{Z} / M \mathbf{Z}) .
$$

We denote by $\left[\begin{array}{l}x \\ y\end{array}\right]_{M}$ the equivalence class in $A_{M}$ of $\left[\begin{array}{l}x \\ y\end{array}\right] \in(\mathbf{Z} / M \mathbf{Z})^{\oplus 2}$ such that $(x, y)=1$ in 
$\mathbf{Z} / M \mathbf{Z}$, or $\left[\begin{array}{l}x \\ y\end{array}\right] \in \mathbf{Z}^{\oplus 2}$ such that $(x, y, M)=1$. In the following, we let $(\mathbf{Z} / M \mathbf{Z})^{\times}$act on $A_{M}$ by

$$
\alpha \cdot\left[\begin{array}{l}
x \\
y
\end{array}\right]_{M}:=\left[\begin{array}{c}
\alpha^{-1} x \\
\alpha y
\end{array}\right]_{M} \quad \text { for } \alpha \in(\mathbf{Z} / M \mathbf{Z})^{\times} .
$$

If $M=M_{1} \times M_{2}$ with mutually coprime positive integers $M_{1}$ and $M_{2}$, there is a natural bijection

$$
A_{M} \stackrel{\sim}{\rightarrow} A_{M_{1}} \times A_{M_{2}}
$$

sending $\left[\begin{array}{l}x \\ y\end{array}\right]_{M}$ to $\left(\left[\begin{array}{l}x \\ y\end{array}\right]_{M_{1}},\left[\begin{array}{l}x \\ y\end{array}\right]_{M_{2}}\right)$, which is of course compatible with the action of

$$
(\mathbf{Z} / M \mathbf{Z})^{\times} \cong\left(\mathbf{Z} / M_{1} \mathbf{Z}\right)^{\times} \times\left(\mathbf{Z} / M_{2} \mathbf{Z}\right)^{\times}
$$

on both sides.

As in Section 1, we fix a positive integer $N$ prime to $p$, and let

$$
N=\prod_{j=1}^{k} q_{j}^{e_{j}}
$$

be its prime decomposition. We write

$$
N_{r}:=N p^{r} \quad \text { for } r \geqslant 1
$$

for simplicity. For any finite set $S$, we denote by $\mathfrak{o}[S]$ the free $\mathfrak{o}$-module generated by the elements of $S, \mathfrak{o}$ being as in Section 1. Then by (2.1.4) above, we have an isomorphism:

$$
\mathfrak{o}\left[A_{N_{r}}\right] \cong\left(\bigotimes_{j=1}^{k} \mathfrak{o}\left[A_{q_{j} e_{j}}\right]\right) \otimes \mathfrak{o}\left[A_{p^{r}}\right]
$$

compatible with the action of

$$
\mathfrak{o}\left[\left(\mathbf{Z} / N_{r} \mathbf{Z}\right)^{\times}\right] \cong\left(\bigotimes_{j=1}^{k} \mathfrak{o}\left[\left(\mathbf{Z} / q_{j}^{e_{j}} \mathbf{Z}\right)^{\times}\right]\right) \otimes \mathfrak{o}\left[\left(\mathbf{Z} / p^{r} \mathbf{Z}\right)^{\times}\right]
$$

We recall that

$$
C_{r}:=\left(\text { the set of cusps of } X_{1}\left(N_{r}\right)(\overline{\mathbf{Q}})\right)
$$

is canonically identified with $A_{N_{r}} /\{ \pm 1\}$, and hence $\mathfrak{o}\left[C_{r}\right]$ with the quotient of $\mathfrak{o}\left[A_{N_{r}}\right]$ by its o-submodule generated by $\left\{x-(-1) \cdot x \mid x \in A_{N_{r}}\right\}$. For our computational purpose, it is rather convenient to work with $\mathfrak{o}\left[A_{N_{r}}\right]$.

We now consider the Hecke operators on $\mathfrak{o}\left[A_{M}\right]$. For this, put

$$
\left\{\begin{array}{l}
U_{\infty}:=\left\{\left[\begin{array}{ll}
1 & * \\
0 & 1
\end{array}\right] \in S L_{2}(\mathbf{Z})\right\} \\
\widetilde{U}_{\infty}:=\left\{\left[\begin{array}{ll}
a & * \\
0 & d
\end{array}\right] \in G L_{2}(\mathbf{Q}) \mid a, d>0\right\} .
\end{array}\right.
$$


Then there is a bijection: $\Gamma_{1}(M) \backslash S L_{2}(\mathbf{Z}) / U_{\infty} \stackrel{\sim}{\rightarrow} A_{M}$ sending an element $\left[\begin{array}{cc}a & b \\ c & d\end{array}\right] \in S L_{2}(\mathbf{Z})$ to $\left[\begin{array}{c}a \\ c\end{array}\right]_{M}$. On the other hand, the natural mapping $S L_{2}(\mathbf{Z}) / U_{\infty} \rightarrow G L_{2}^{+}(\mathbf{Q}) / \widetilde{U}_{\infty}$ is bijective. Consequently, we may identify $\mathfrak{o}\left[A_{M}\right]$ with $\mathfrak{o}\left[\Gamma_{1}(M) \backslash G L_{2}^{+}(\mathbf{Q}) / \widetilde{U}_{\infty}\right]$. We can then consider the action of the abstract Hecke algebra attached to $\Gamma_{1}(M)$ and $G L_{2}^{+}(\mathbf{Q})$ (cf. Shimura [18], 3.1) on this module. Namely, for any $g \in G L_{2}^{+}(\mathbf{Q})$, we let a double coset

$$
\Gamma_{1}(M) g \Gamma_{1}(M)=\coprod_{i} \Gamma_{1}(M) g_{i}
$$

act as: (the class of $\gamma \in G L_{2}^{+}(\mathbf{Q})$ in $\left.A_{M}\right) \mapsto \sum_{i}$ (the class of $g_{i} \gamma$ ).

For any prime number $l$, we denote by $T^{*}(l)$ the endomorphism of $\mathfrak{o}\left[A_{M}\right]$ determined by the double coset containing $\left[\begin{array}{cc}1 & 0 \\ 0 & l\end{array}\right]$, to be consistent with the terminology of [15], 3.4. A simple calculation shows that the action of $T^{*}(l)$ on $\left[\begin{array}{l}a \\ c\end{array}\right]_{M}(a, c \in \mathbf{Z},(a, c)=1)$ is given by the following formulas:

- When $l$ does not divide $M$, let $l^{\prime}$ be an integer such that $l l^{\prime} \equiv 1 \bmod M$. Then we have:

$$
T^{*}(l)\left[\begin{array}{l}
a \\
c
\end{array}\right]_{M}=l\left[\begin{array}{l}
a \\
l c
\end{array}\right]_{M}+\left[\begin{array}{c}
l^{\prime} a \\
c
\end{array}\right]_{M} .
$$

- When $l$ divides $M$ but does not divide $c$, let $i_{0}$ be the unique integer such that $0 \leqslant i_{0} \leqslant l-1$ and $a+i_{0} c \equiv 0 \bmod l$. Then

$$
T^{*}(l)\left[\begin{array}{l}
a \\
c
\end{array}\right]_{M}=\sum_{\substack{i=0 \\
i \neq i_{0}}}^{l-1}\left[\begin{array}{c}
a+i c \\
l c
\end{array}\right]_{M}+\left[\begin{array}{c}
\left(a+i_{0} c\right) / l \\
c
\end{array}\right]_{M} .
$$

- When $l$ divides both $M$ and $c$,

$$
T^{*}(l)\left[\begin{array}{l}
a \\
c
\end{array}\right]_{M}=\sum_{i=0}^{l-1}\left[\begin{array}{c}
a+i c \\
l c
\end{array}\right]_{M}
$$

Via the canonical mapping: $\mathfrak{o}\left[A_{N_{r}}\right] \rightarrow \mathfrak{o}\left[C_{r}\right]$ above, these operators induce the operators on $\mathfrak{o}\left[C_{r}\right]$ denoted by the same symbols in [15], loc. cit.

\subsection{Eisenstein elements in cuspidal groups}

We fix Dirichlet characters $\theta$ and $\psi$ of conductors $u$ and $v$, respectively, satisfying the same conditions as in Section 1.4. We assume that $u v=N$ or $N p$, and that $\mathfrak{o}$ contains all the values of $\theta$ and $\psi$.

For each prime factor $q_{j}$ of $N$, we set

$$
\left\{\begin{array}{l}
\operatorname{ord}_{q_{j}}(u):=f_{j} \\
\operatorname{ord}_{q_{j}}(v):=h_{j}
\end{array}\right.
$$

so that $\operatorname{ord}_{q_{j}}(N)=e_{j}=f_{j}+h_{j}$. 
DEFINITION (2.2.2). - Denote by $u_{1}$ the least common multiple of $u$ and $p$. Identifying the both sides of (2.1.7), we define $\mathbf{e}_{r}^{\prime} \in \mathfrak{o}\left[A_{N_{r}}\right]$ by

$$
\mathbf{e}_{r}^{\prime}:=\sum_{\alpha \in(\mathbf{Z} / v \mathbf{Z})^{\times}} \sum_{\beta \in\left(\mathbf{Z} / u_{1} \mathbf{Z}\right)^{\times}} \theta(\beta)^{-1} \psi(\alpha)\left(\bigotimes_{j=1}^{k}\left[\begin{array}{c}
\alpha \\
\beta q_{j}^{h_{j}}
\end{array}\right]_{q_{j}^{e_{j}}}\right) \otimes\left[\begin{array}{c}
0 \\
\omega(\beta)
\end{array}\right]_{p^{r}} .
$$

It is easy to see that $\mathbf{e}_{r}^{\prime}$ is a well-defined element. We again write $e^{*}$ for Hida's idempotent attached to $T^{*}(p)$ acting on $\mathfrak{o}\left[A_{N_{r}}\right]$. For the same reason as [15], (4.3.4), if we denote by $D_{r}^{\prime}$ the $\mathfrak{o}$-submodule of $\mathfrak{o}\left[A_{N_{r}}\right]$ generated by $\left[\begin{array}{c}a \\ c\end{array}\right]_{N_{r}}$ with $p \mid c$, then

$$
e^{*} D_{r}^{\prime}=\{0\} \quad \text { and } \quad e^{*} \mathfrak{o}\left[A_{N_{r}}\right] \stackrel{\sim}{\rightarrow} \mathfrak{o}\left[A_{N_{r}}\right] / D_{r}^{\prime} .
$$

Now we have that $\mathbf{e}_{r}^{\prime}$ is non-zero. In fact, it is easy to see that

$$
\left(\bigotimes_{j=1}^{k}\left[\begin{array}{c}
\alpha \\
\beta q_{j}^{h_{j}}
\end{array}\right]_{q_{j}^{e_{j}}}\right) \otimes\left[\begin{array}{c}
0 \\
\omega(\beta)
\end{array}\right]_{p^{r}} \in A_{N_{r}}
$$

are all different when $\alpha \in(\mathbf{Z} / v \mathbf{Z})^{\times}$and $\beta \in\left(\mathbf{Z} / u_{1} \mathbf{Z}\right)^{\times}$vary. Consequently, by the remark above, we see that $e^{*} \mathbf{e}_{r}^{\prime}$ is also non-zero.

In the following, we denote by $\iota$ the inclusion mapping of $\left(\mathbf{Z} / N_{r} \mathbf{Z}\right)^{\times}$into $\mathfrak{o}\left[\left(\mathbf{Z} / N_{r} \mathbf{Z}\right)^{\times}\right]$, the latter acting on $\mathfrak{o}\left[A_{N_{r}}\right]$ by the rule (2.1.3). It follows from the definition that

$$
\iota(q) \mathbf{e}_{r}^{\prime}=(\theta \psi)(q) \iota(\langle q\rangle) \mathbf{e}_{r}^{\prime}
$$

for any integer $q$ prime to $N p$, where $\iota(\langle q\rangle) \in \mathfrak{o}\left[U_{1} / U_{r}\right] \subset \mathfrak{o}\left[\left(\mathbf{Z} / N_{r} \mathbf{Z}\right)^{\times}\right]$.

PROPOSITION (2.2.5). - The notation being as above, we have

$$
T^{*}(l)\left(e^{*} \mathbf{e}_{r}^{\prime}\right)= \begin{cases}(\theta(l) l \iota(\langle l\rangle)+\psi(l))\left(e^{*} \mathbf{e}_{r}^{\prime}\right) & \text { if } l \neq p \\ \psi(p)\left(e^{*} \mathbf{e}_{r}^{\prime}\right) & \text { if } l=p\end{cases}
$$

Proof. - We first treat the case where $l \mid N$ so that $l=q_{j_{0}}$ with some index $j_{0}$. Take $\alpha \in(\mathbf{Z} / v \mathbf{Z})^{\times}$and $\beta \in\left(\mathbf{Z} / u_{1} \mathbf{Z}\right)^{\times}$, and choose coprime integers $a$ and $c$ such that $a \equiv \alpha$ and $c \equiv \beta q_{j}^{h_{j}} \bmod q_{j}^{e_{j}}(1 \leqslant j \leqslant k) ; a \equiv 0$ and $c \equiv \omega(\beta) \bmod p^{r}$, so that

$$
\left[\begin{array}{l}
a \\
c
\end{array}\right]_{N_{r}}=\left(\bigotimes_{j=1}^{k}\left[\begin{array}{c}
\alpha \\
\beta q_{j}^{h_{j}}
\end{array}\right]_{q_{j}^{e_{j}}}\right) \otimes\left[\begin{array}{c}
0 \\
\omega(\beta)
\end{array}\right]_{p^{r}} .
$$

Assume that $h_{j_{0}}=0$. In this case, we have $f_{j_{0}}=e_{j_{0}}$ and $l=q_{j_{0}} \nmid c$. Using the same notation as in (2.1.11), we have

$$
\sum_{\substack{i=0 \\
i \neq i_{0}}}^{l-1}\left[\begin{array}{c}
a+i c \\
l c
\end{array}\right]_{N_{r}}=\left(\bigotimes_{j \neq j_{0}}\left[\begin{array}{c}
\alpha \\
l \beta q_{j}^{h_{j}}
\end{array}\right]_{q_{j}}\right) \otimes\left(\sum_{i=1}^{q_{j_{0}}-1}\left[\begin{array}{c}
i \\
\beta q_{j_{0}}
\end{array}\right]_{q_{j_{0}} e_{j_{0}}}\right) \otimes\left[\begin{array}{c}
0 \\
l \omega(\beta)
\end{array}\right]_{p^{r}}=: X_{\alpha, \beta} .
$$

We clearly have $X_{\alpha, \beta}=X_{\alpha, \beta^{\prime}}$ whenever $\beta \equiv \beta^{\prime} \bmod u_{1} / q_{j_{0}}$, and so

$$
\sum_{\alpha \in(\mathbf{Z} / v \mathbf{Z})^{\times}} \sum_{\beta \in\left(\mathbf{Z} / u_{1} \mathbf{Z}\right)^{\times}} \theta(\beta)^{-1} \psi(\alpha) X_{\alpha, \beta}=0 .
$$


On the other hand, we have

$$
\left[\begin{array}{c}
\left(a+i_{0} c\right) / l \\
c
\end{array}\right]_{N_{r}}=\left(\bigotimes_{j \neq j_{0}}\left[\begin{array}{c}
l^{\prime} \alpha \\
\beta q_{j}^{h_{j}}
\end{array}\right]_{q_{j}^{e_{j}}}\right) \otimes\left[\begin{array}{l}
0 \\
\beta
\end{array}\right]_{q_{j_{0}}^{e_{j}}} \otimes\left[\begin{array}{c}
0 \\
\omega(\beta)
\end{array}\right]_{p^{r}}
$$

where $l l^{\prime} \equiv 1 \bmod N$. We conclude that $T^{*}(l) \mathbf{e}_{r}^{\prime}=\psi(l) \mathbf{e}_{r}^{\prime}$, which gives our formula, since $e^{*}$ commutes with $T^{*}(l)$, and $\theta(l)=0$.

Next assume that $h_{j_{0}}>0$, i.e. $l=q_{j_{0}} \mid c$. In this case, we apply (2.1.12). For $\alpha, \beta$ and $\left[\begin{array}{c}a \\ c\end{array}\right]_{N_{r}}$ as above, we have

$$
T^{*}(l)\left[\begin{array}{l}
a \\
c
\end{array}\right]_{N_{r}}=\left(\bigotimes_{j \neq j_{0}}\left[\begin{array}{c}
\alpha \\
l \beta q_{j}^{h_{j}}
\end{array}\right]_{q_{j}^{e_{j}}}\right) \otimes\left(\sum_{i=0}^{q_{j_{0}}-1}\left[\begin{array}{c}
\alpha+i \beta q_{j_{0}}^{h_{j_{0}}} \\
\beta q_{j_{0}}^{h_{j_{0}}+1}
\end{array}\right]_{q_{j_{0}}^{e_{0}}}\right) \otimes\left[\begin{array}{c}
0 \\
l \omega(\beta)
\end{array}\right]_{p^{r}}=: Y_{\alpha, \beta}
$$

When $h_{j_{0}}=e_{j_{0}}$, the sum in the middle term is $q_{j_{0}}\left[\begin{array}{c}\alpha \\ 0\end{array}\right]_{q_{j_{0}}}^{e_{j_{0}}}$, and we easily obtain

$$
T^{*}(l) \mathbf{e}_{r}^{\prime}=\theta(l) l \iota(\langle l\rangle) \mathbf{e}_{r}^{\prime} .
$$

On the other hand, when $h_{j_{0}} \neq e_{j_{0}}$ so that $f_{j_{0}}>0$ and $\theta(l)=\psi(l)=0$, we see that $\beta \equiv \beta^{\prime}$ mod $u_{1} / q_{j_{0}}$ implies $Y_{\alpha, \beta}=Y_{\alpha, \beta^{\prime}}$. We therefore conclude that $T^{*}(l) \mathbf{e}_{r}^{\prime}=0$.

We have thus settled the case where $l \mid N$. When $l \nmid N p$, the proof is similar and in fact simpler. Finally assume that $l=p$. It is in this case we have to consider $e^{*} \mathbf{e}_{r}^{\prime}$ rather than $\mathbf{e}_{r}^{\prime}$ : Using the same convention as in (2.1.11), (2.2.3) implies that

$$
T^{*}(p) e^{*}\left[\begin{array}{l}
a \\
c
\end{array}\right]_{N_{r}}=e^{*}\left[\begin{array}{c}
\left(a+i_{0} c\right) / p \\
c
\end{array}\right]_{N_{r}}
$$

whenever $p \nmid c$. One then easily derives $T^{*}(p)\left(e^{*} \mathbf{e}_{r}^{\prime}\right)=\psi(p)\left(e^{*} \mathbf{e}_{r}^{\prime}\right)$.

Definition (2.2.6). - We denote by $\mathbf{e}_{r}$ the image of $\mathbf{e}_{r}^{\prime}$ in $\mathfrak{o}\left[C_{r}\right]$.

\subsection{Eisenstein elements in $\Lambda$-adic cuspidal groups}

We keep the notation of the previous subsection. When $r \geqslant 1$ varies, there are natural surjections $A_{N_{s}} \rightarrow A_{N_{r}}$ sending $\left[\begin{array}{c}a \\ c\end{array}\right]_{N_{s}}$ to $\left[\begin{array}{c}a \\ c\end{array}\right]_{N_{r}}$ for $s \geqslant r$. We can therefore form the projective limits:

$$
\left\{\begin{array}{l}
\mathfrak{o} \llbracket A_{N p^{\infty}} \rrbracket:=\lim _{r \geqslant 1} \mathfrak{o}\left[A_{N_{r}}\right]=\mathfrak{o}\left[A_{N}\right] \otimes \mathfrak{o} \llbracket A_{p^{\infty}} \rrbracket, \\
\mathfrak{o} \llbracket C_{\infty} \rrbracket:=\lim _{r \geqslant 1} \mathfrak{o}\left[C_{r}\right] .
\end{array}\right.
$$

These are modules over $\mathfrak{o} \llbracket \lim _{r} \geqslant 1\left(\mathbf{Z} / N_{r} \mathbf{Z}\right)^{\times} \rrbracket$ in the manner described in Section 2.1. Especially they are modules over $\Lambda_{\mathfrak{o}}$. As in [15], we set

$$
\mathcal{C}_{p}(N)_{\mathfrak{o}}:=e^{*} \mathfrak{o} \llbracket C_{\infty} \rrbracket .
$$

Via the action of Hecke operators described in Section 2.1, we can consider $\mathcal{C}_{p}(N)_{0}$ as an $e^{*} \mathcal{H}^{*}(N ; \mathfrak{o})$-module $([15], 4.3)$, the resulting $\mathfrak{o} \llbracket \varliminf_{r \geqslant 1}\left(\mathbf{Z} / N_{r} \mathbf{Z}\right)^{\times} \rrbracket$-module structure being the one described above. We then recall that $\mathcal{C}_{p}(N)_{\mathfrak{o}}$ is a free $\Lambda_{\mathfrak{o}}$-module of finite rank (loc. cit.). 
It is clear from the definitions that $\mathbf{e}_{r}^{\prime}$ and $\mathbf{e}_{r}$ give rise to elements of the projective limits above, and we set

$$
\left\{\begin{array}{l}
\mathbf{e}_{\infty}^{\prime}:=\left(\mathbf{e}_{r}^{\prime}\right)_{r \geqslant 1} \in \mathfrak{o} \llbracket A_{N p^{\infty}} \rrbracket \\
\mathbf{e}_{\infty}:=\left(\mathbf{e}_{r}\right)_{r \geqslant 1} \in \mathfrak{o} \llbracket C_{\infty} \rrbracket .
\end{array}\right.
$$

We now assume that $p$ does not divide $\varphi(N)$. Set

$$
\chi=\theta \psi \text {. }
$$

For any $\mathfrak{o}\left[\left(\mathbf{Z} / N_{r} \mathbf{Z}\right)^{\times}\right]$-module $M$, we denote by $M^{(\chi)}$ its $\chi$-eigenspace, i.e. the maximum direct summand of $M$ on which $\left(\mathbf{Z} / N_{r} \mathbf{Z}\right)^{\times}$acts through the (possibly imprimitive) character $\chi$. Then since $\chi$ is even, we have canonical isomorphisms:

$$
\left\{\begin{array}{l}
\mathfrak{o}\left[A_{N_{r}}\right]^{(\chi)} \cong \mathfrak{o}\left[C_{r}\right]^{(\chi)} \\
\mathfrak{o} \llbracket A_{N p^{\infty}} \rrbracket^{(\chi)} \cong \mathfrak{o} \llbracket C_{\infty} \rrbracket^{(\chi)}
\end{array}\right.
$$

We see from (2.2.4) that $\mathbf{e}_{\infty}$ lies in $\mathfrak{o} \llbracket C_{\infty} \rrbracket^{(\chi)}$.

THEOREM (2.3.6). - Assume that $p \nmid \varphi(N)$. Then $\Lambda_{\mathfrak{o}}\left(e^{*} \mathbf{e}_{\infty}\right)$ is a free $\Lambda_{\mathfrak{o}}$-module of rank one, and it is a direct summand of the free $\Lambda_{\mathfrak{o}}$-module $\mathcal{C}_{p}(N)_{\mathfrak{o}}$ or $\mathcal{C}_{p}(N)_{\mathfrak{o}}^{(\chi)}$. Moreover, it coincides with the kernel of the Eisenstein ideal defined in (1.5.1):

$$
\Lambda_{\mathfrak{o}}\left(e^{*} \mathbf{e}_{\infty}\right)=\mathcal{C}_{p}(N)_{\mathfrak{o}}\left[\mathcal{I}^{*}(\theta, \psi)\right]
$$

Proof. - We fix an $r \geqslant 1$ for the moment. Let $M_{r}$ be the $\mathfrak{o}$-submodule of $\mathfrak{o}\left[A_{N_{r}}\right]$ generated by all

$$
\left(\bigotimes_{j=1}^{k}\left[\begin{array}{c}
\alpha \\
\beta q_{j}^{h_{j}}
\end{array}\right]_{q_{j}^{e_{j}}}\right) \otimes\left[\begin{array}{c}
0 \\
\omega(\beta) \gamma
\end{array}\right]_{p^{r}}
$$

for $\alpha \in(\mathbf{Z} / v \mathbf{Z})^{\times}, \beta \in\left(\mathbf{Z} / u_{1} \mathbf{Z}\right)^{\times}$and $\gamma \in U_{1} / U_{r}$. As noted in the previous subsection, these are well-defined elements of $A_{N_{r}}$, and are in fact all different. Evidently, as an $\mathfrak{o}\left[\left(\mathbf{Z} / N_{r} \mathbf{Z}\right)^{\times}\right]$module, $\mathfrak{o}\left[A_{N_{r}}\right]$ is a direct sum of $M_{r}$ and the $\mathfrak{o}$-submodule generated by the elements of $A_{N_{r}}$ not contained in $M_{r}$.

On the other hand, we can make $M_{r}$ into a module over $(\mathbf{Z} / v \mathbf{Z})^{\times} \times\left(\mathbf{Z} / u_{1} \mathbf{Z}\right)^{\times} \times\left(U_{1} / U_{r}\right)$ by letting an element $\left(\alpha^{\prime}, \beta^{\prime}, \gamma^{\prime}\right)$ in this group send the element above to

$$
\left(\bigotimes_{j=1}^{k}\left[\begin{array}{c}
\alpha^{\prime} \alpha \\
\beta^{\prime} \beta q_{j}^{h_{j}}
\end{array}\right]_{q_{j}^{e_{j}}}\right) \otimes\left[\begin{array}{c}
0 \\
\omega\left(\beta^{\prime} \beta\right) \gamma^{\prime} \gamma
\end{array}\right]_{p^{r}} .
$$

It is then clear that $M_{r}$ is a free $\mathfrak{o}\left[(\mathbf{Z} / v \mathbf{Z})^{\times} \times\left(\mathbf{Z} / u_{1} \mathbf{Z}\right)^{\times} \times\left(U_{1} / U_{r}\right)\right]$-module of rank one with a basis

$$
\mathbf{p}_{r}:=\left(\bigotimes_{j=1}^{k}\left[\begin{array}{c}
1 \\
q_{j}^{h_{j}}
\end{array}\right]_{q_{j}^{e_{j}}}\right) \otimes\left[\begin{array}{l}
0 \\
1
\end{array}\right]_{p^{r}} .
$$

By our assumption, the order $g$ of the group $(\mathbf{Z} / v \mathbf{Z})^{\times} \times\left(\mathbf{Z} / u_{1} \mathbf{Z}\right)^{\times}$is prime to $p$. Therefore, the element $\mathbf{e}_{r}^{\prime}$ is nothing but the one obtained by applying the idempotent attached to the character $\psi^{-1} \times \theta:(\alpha, \beta) \mapsto \theta(\beta) \psi^{-1}(\alpha)$ of $(\mathbf{Z} / v \mathbf{Z})^{\times} \times\left(\mathbf{Z} / u_{1} \mathbf{Z}\right)^{\times}$, to a basis $g \mathbf{p}_{r}$. Thus 
$\mathfrak{o}\left[U_{1} / U_{r}\right] \mathbf{e}_{r}^{\prime}=M_{r}^{\left(\psi^{-1} \times \theta\right)}$, which is an $\mathfrak{o}\left[U_{1} / U_{r}\right]$-module direct summand of $\mathfrak{o}\left[A_{N_{r}}\right]$, and free of rank one over $\mathfrak{o}\left[U_{1} / U_{r}\right]$. Note that $M_{r}^{\left(\psi^{-1} \times \theta\right)}$ is contained in $M_{r}^{(\chi)}$ in the previous sense.

We deduce from this and (2.2.3) that $\mathfrak{o}\left[U_{1} / U_{r}\right]\left(e^{*} \mathbf{e}_{r}\right)$ is free of rank one over $\mathfrak{o}\left[U_{1} / U_{r}\right]$, and that it is a direct summand of the $\mathfrak{o}\left[U_{1} / U_{r}\right]$-module $e^{*} \mathfrak{o}\left[C_{r}\right]^{(\chi)}$. The first assertion follows from this.

Now it follows from (2.2.4) and (2.2.5) that $e^{*} \mathbf{e}_{\infty} \in \mathcal{C}_{p}(N)_{\mathfrak{o}}\left[\mathcal{I}^{*}(\theta, \psi)\right]$. Let $\mathcal{L}_{K}$ be the quotient field of $\Lambda_{\mathfrak{o}}$. Then since $\mathcal{C}_{p}(N)_{\mathfrak{o}} / \Lambda_{\mathfrak{o}}\left(e^{*} \mathbf{e}_{r}\right)$ is free over $\Lambda_{\mathfrak{o}}$, it is enough to show that $\left(\mathcal{C}_{p}(N)_{\mathfrak{o}} \otimes_{\Lambda_{\mathfrak{o}}} \mathcal{L}_{K}\right)\left[\mathcal{I}^{*}(\theta, \psi)\right]$ is a one-dimensional $\mathcal{L}_{K}$-vector space, to finish the proof. For this, one easily reduces the problem to the case where $K$ contains all the roots of unity. In this case, by the main results of [15] and [16] (cf. also (2.4.6) below), $\mathcal{C}_{p}(N)_{\mathfrak{o}} \otimes_{\Lambda_{\mathfrak{o}}} \mathcal{L}_{K}$ is canonically isomorphic to the $\mathcal{L}_{K}$-vector space generated by the $\Lambda$-adic Eisenstein series given in Section 1.4, and via this isomorphism, $T^{*}(-)$ on the former corresponds to $T(-)$ on the latter. Thus by (1.4.8), the space above is one-dimensional.

\subsection{Residue mapping for $\Lambda$-adic modular forms}

Assume that $\mathfrak{o}$ contains all the roots of unity. We first recall some results from our previous works. We write $\Gamma_{r}$ for $\Gamma_{1}\left(N p^{r}\right)$, and $S_{2}\left(\Gamma_{r} ; \mathfrak{o}\right)$ (resp. $\left.M_{2}\left(\Gamma_{r} ; \mathfrak{o}\right)\right)$ for the space of cusp forms (resp. modular forms) of weight 2 with respect to $\Gamma_{r}$ with coefficients in $\mathfrak{o}$. We then denote by $S_{2}^{*}\left(\Gamma_{r} ; \mathfrak{o}\right)$ (resp. $M_{2}^{*}\left(\Gamma_{r} ; \mathfrak{o}\right)$ ) the space obtained from the above by applying the operator “| $\tau_{r}$ " with $\tau_{r}:=\left[\begin{array}{cc}0 & -1 \\ N_{r} & 0\end{array}\right]$. (See [14], (2.1.3), (2.3.4) and [15], (1.5.8), (2.2.1) for more details.) Denoting by $e^{*}$ Hida's idempotent attached to $T^{*}(p)$ acting on the latter spaces, we recall that there is an exact sequence for each $r \geqslant 1$

$$
0 \rightarrow e^{*} S_{2}^{*}\left(\Gamma_{r} ; \mathfrak{o}\right) \rightarrow e^{*} M_{2}^{*}\left(\Gamma_{r} ; \mathfrak{o}\right) \stackrel{\operatorname{Res}_{r}}{\rightarrow} e^{*} \mathfrak{o}\left[C_{r}\right] \rightarrow 0
$$

where $\operatorname{Res}_{r}$ sends $f \in e^{*} M_{2}^{*}\left(\Gamma_{r} ; \mathfrak{o}\right)$ to the formal sum obtained by taking residues at cusps:

$$
\operatorname{Res}_{r}(f):=\sum_{c \in C_{r}} \operatorname{Res}_{c}\left(\omega_{f}\right) \cdot c \quad \text { with } \omega_{f}:=f \frac{d q}{q} .
$$

Indeed, in [15], 4.4, we have established this for certain subspaces, and the general case is covered by the argument in [16], 2.2.

When $s \geqslant r \geqslant 1$, we have the following commutative diagram:

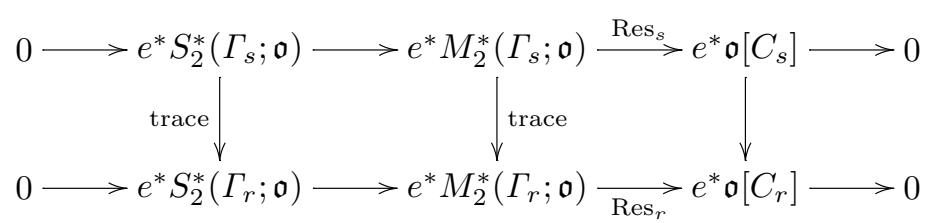

where the left two vertical arrows are the natural trace mappings (i.e. they correspond to the traces of differentials via $f \leftrightarrow \omega_{f}$ ), and the right vertical one is induced from the projection: $C_{s} \rightarrow C_{r}$. Then taking the projective limit, using the same terminology as [14], (2.3.5) and [15], (2.2.2), we obtain the exact sequence

$$
0 \rightarrow e^{*} \mathfrak{S}_{2}^{*}(N ; \mathfrak{o}) \rightarrow e^{*} \mathfrak{M}_{2}^{*}(N ; \mathfrak{o}) \stackrel{\operatorname{Res}_{\infty}}{\longrightarrow} \mathcal{C}_{p}(N)_{\mathfrak{o}} \rightarrow 0
$$


(To check the surjectivity of $\operatorname{Res}_{\infty}$ directly, it is enough to show that this mapping is surjective when reduced modulo $T \Lambda_{\mathfrak{o}}$, since its domain and range are both finitely generated free $\Lambda_{\mathfrak{o}}$-modules ([15], (2.4.10)). By [15], (2.5.4) and (4.3.14), this amounts to the surjectivity of $\operatorname{Res}_{1}$.)

On the other hand, there are canonical isomorphisms:

$$
\left\{\begin{array}{l}
e^{*} \mathfrak{S}_{2}^{*}(N ; \mathfrak{o}) \cong e S\left(N ; \Lambda_{\mathfrak{o}}\right), \\
e^{*} \mathfrak{M}_{2}^{*}(N ; \mathfrak{o}) \cong e M\left(N ; \Lambda_{\mathfrak{o}}\right)
\end{array}\right.
$$

([14], (2.3.6), [15], (2.2.3)). Combining these with (2.4.4), we obtain the exact sequence

$$
0 \rightarrow e S\left(N ; \Lambda_{\mathfrak{o}}\right) \rightarrow e M\left(N ; \Lambda_{\mathfrak{o}}\right) \stackrel{\text { Res }}{\rightarrow} \mathcal{C}_{p}(N)_{\mathfrak{o}} \rightarrow 0
$$

We call Res the residue mapping for $\Lambda$-adic modular forms. Let us make this mapping explicit: An element $\mathcal{F} \in e M\left(N ; \Lambda_{\mathfrak{o}}\right)$ corresponds, via $(2.4 .5)$, to $\left(f_{r}\right)_{r \geqslant 1} \in e^{*} \mathfrak{M}_{2}^{*}(N ; \mathfrak{o})$ defined by

$$
f_{r}=\frac{1}{p^{r-1}}\left(\sum_{\varepsilon \in \widehat{U_{1} / U_{r}}} \mathcal{F}_{\varepsilon, 0}\left|T(p)^{-r}\right| \tau_{r}^{-1}\right)
$$

where $\widehat{U_{1} / U_{r}}$ is the set of $\left(\overline{\mathbf{Q}}^{\times}\right.$-valued $)$characters of $U_{1} / U_{r}$. Thus if we set

$$
g_{r}:=\frac{1}{p^{r-1}}\left(\sum_{\varepsilon \in \widehat{U_{1} / U_{r}}} \mathcal{F}_{\varepsilon, 0} \mid T(p)^{-r}\right)
$$

we see that the composite of $e M\left(N ; \Lambda_{\mathfrak{o}}\right) \stackrel{\text { Res }}{\rightarrow} \mathcal{C}_{p}(N)_{\mathfrak{o}} \rightarrow e^{*} \mathfrak{o}\left[C_{r}\right]$ sends $\mathcal{F}$ to

$$
\sum_{c \in C_{r}} \operatorname{Res}_{\tau_{r}(c)}\left(\omega_{g_{r}}\right) \cdot c
$$

Now let $\theta$ and $\psi$ be Dirichlet characters as in Section 2.2, and assume that $(\theta, \psi) \neq\left(\omega^{-2}, \mathbf{1}\right)$. Since Res commutes with the Hecke operators in the sense that $T(-)$ on the domain commutes with $T^{*}(-)$ on the range, we see that $\operatorname{Res}(\mathcal{E}(\theta, \psi))$ lies in $\mathcal{C}_{p}(N)_{\mathfrak{o}}\left[\mathcal{I}^{*}(\theta, \psi)\right]$, in general. The following is the main result of this section:

THEOREM (2.4.10). - Let the notation and the assumption be as in (2.3.6) with

$$
(\theta, \psi) \neq\left(\omega^{-2}, \mathbf{1}\right)
$$

Then the image of $\mathcal{E}(\theta, \psi) \in e M\left(N ; \Lambda_{\mathfrak{o}}\right)$ under $\operatorname{Res}$ is a multiple of $A(T ; \theta, \psi)\left(e^{*} \mathbf{e}_{\infty}\right)$ by a unit in $\Lambda_{\mathfrak{o}}$. Here, $A(T ; \theta, \psi)$ is the power series defined by (1.5.4).

To prove this theorem, we will study the classical Eisenstein series in the next subsection.

\subsection{Classical Eisenstein series of weight 2}

Let $M$ be a positive integer. In [3], Hecke studied (not necessarily holomorphic) Eisenstein series for the principal congruence subgroup $\Gamma(M)$. When the weight is 2 , they are given by 


$$
\begin{aligned}
G_{2}\left(z ; a_{1}, a_{2}, M\right):= & \left.\sum_{m_{i} \equiv a_{i}(M)}^{\prime} \frac{1}{\left(m_{1} z+m_{2}\right)^{2}\left|m_{1} z+m_{2}\right|^{s}}\right|_{s=0} \\
= & \frac{-\pi}{M^{2} y}+\delta^{\prime}\left(\frac{a_{1}}{M}\right) \sum_{m_{2} \equiv a_{2}(M)}^{\prime} \frac{1}{m_{2}^{2}} \\
& -\frac{4 \pi^{2}}{M^{2}} \sum_{\substack{m m_{1}>0 \\
m_{1} \equiv a_{1}(M)}}|m| \mathrm{e}^{2 \pi i \frac{a_{2} m}{M}} \mathrm{e}^{2 \pi i \frac{m m_{1}}{M} z}
\end{aligned}
$$

Here, $a_{1}$ and $a_{2}$ are integers; $z=x+y i$ is a variable on the complex upper half plane; and $\delta^{\prime}\left(a_{1} / M\right)$ is equal to 1 (resp. 0 ) when $a_{1} / M$ is an integer (resp. otherwise). The superscripts prime for the sum indicate the exclusion of the term $m_{1}=m_{2}=0$ or $m_{2}=0$. For

$$
\gamma=\left[\begin{array}{ll}
a & b \\
c & d
\end{array}\right] \in S L_{2}(\mathbf{Z})
$$

the Eisenstein series above satisfy the transformation law

$$
\begin{aligned}
G_{2}\left(z ; a_{1}, a_{2}, M\right) \mid \gamma & :=\frac{G_{2}\left(\frac{a z+b}{c z+d} ; a_{1}, a_{2}, M\right)}{(c z+d)^{2}} \\
& =G_{2}\left(z ; a a_{1}+c a_{2}, b a_{1}+d a_{2}, M\right) .
\end{aligned}
$$

Now let $\theta$ and $\psi$ be Dirichlet characters of conductors $u$ and $v$, respectively. We assume that $\theta \psi$ is even, and that one of $\theta$ or $\psi$ is non-trivial. In the following, we set $M:=u v$. The Eisenstein series

$$
E_{2}(\theta, \psi):=\delta(\psi) L(1-2, \theta)+\sum_{n=1}^{\infty}\left(\sum_{0<t \mid n} \theta(t) \psi\left(\frac{n}{t}\right) t\right) q^{n}
$$

(notation as in (1.4.3)) for $\Gamma_{1}(M)$ can be expressed in terms of Hecke's Eisenstein series above by the following formula of Hida:

LEMMA (2.5.3) (cf. [6], p. 577). - The series

$$
E_{2}^{\prime}(\theta, \psi):=\sum_{\alpha=1}^{v} \sum_{\beta=1}^{M} \psi(\alpha) \bar{\theta}(\beta) G_{2}(z ; \alpha u, \beta, M)
$$

is equal to

$$
-2 \tau(\bar{\theta})\left(\frac{2 \pi}{u}\right)^{2} E_{2}(\theta, \psi),
$$

where $\tau(\bar{\theta}):=\sum_{a=1}^{u} \bar{\theta}(a) \mathrm{e}^{2 \pi i a / u}$ is the usual Gauss sum.

Proof. - Hida noted that $E_{2}^{\prime}(\theta, \psi)$ is a constant multiple of $E_{2}(\theta, \psi)$, and there is no doubt that he knew its explicit form. Since this is not stated in [6], we give the calculation: We consider the "non-constant term" of $E_{2}^{\prime}(\theta, \psi)$ divided by $-4 \pi^{2} / M^{2}$. We see from (2.5.1) that it is equal to

$$
\sum_{\alpha=1}^{v} \sum_{\beta=1}^{M} \psi(\alpha) \bar{\theta}(\beta) \sum_{\substack{m k>0 \\ k \equiv \alpha u(M)}}|m| \mathrm{e}^{2 \pi i \frac{\beta m}{M}} \mathrm{e}^{2 \pi i \frac{m k}{M} z}
$$


Here, $k$ must be divisible by $u$, and thus putting $k=r u$, this reads:

$$
\sum_{\beta=1}^{M} \sum_{m r>0} \psi(r) \bar{\theta}(\beta)|m| \mathrm{e}^{2 \pi i \frac{\beta m}{M}} \mathrm{e}^{2 \pi i \frac{m r}{v} z}
$$

When $v \nmid m$, the sum $\sum_{\beta=1}^{M} \bar{\theta}(\beta) \mathrm{e}^{2 \pi i \frac{\beta m}{M}}$ vanishes, and hence we only need to consider the terms with $m$ divisible by $v$. Then setting $m=v s$, we see that the above is equal to

$$
\sum_{n=1}^{\infty}\left(\sum_{n=r s} \psi(r) \theta(s) v \tau(\bar{\theta})|v s|\right) \mathrm{e}^{2 \pi i n z}
$$

Since $\psi \theta$ is even, this is equal to the non-constant term of $2 v^{2} \tau(\bar{\theta}) E_{2}(\theta, \psi)$. (Of course, one easily checks that the "constant terms" also agree: See the proposition below.)

We next want to calculate the constant terms of $E_{2}(\theta, \psi)$ at various cusps. For this, we take and fix $\gamma=\left[\begin{array}{ll}a & b \\ c & d\end{array}\right] \in S L_{2}(\mathbf{Z})$, so that $\gamma(i \infty)=a / c \in \mathbf{P}^{1}(\mathbf{Q})$ gives the cusp $\left[\begin{array}{l}a \\ c\end{array}\right]_{M} \bmod \{ \pm 1\}$ in the notation of Section 2.1 .

Proposition (2.5.5). - The constant term of the q-expansion of $E_{2}(\theta, \psi) \mid \gamma$ is 0 if $u \nmid c$. When $u \mid c$, it is given by

$$
\frac{1}{2} \frac{\tau(\psi \bar{\theta})}{\tau(\bar{\theta})}\left(\frac{\operatorname{cond}(\theta)}{\operatorname{cond}(\psi \bar{\theta})}\right)^{2} \psi\left(-\frac{c}{u}\right) \bar{\theta}(a)\left(\prod_{\substack{l \mid M \\ l \nmid \operatorname{cond}(\theta \bar{\psi})}}\left(1-(\psi \bar{\theta})(l) l^{-2}\right)\right) \times L(1-2, \theta \bar{\psi})
$$

where the product ranges over all primes satisfying the indicated conditions.

Proof. - We compute the constant term of $E_{2}^{\prime}(\theta, \psi) \mid \gamma$. By (2.5.2), we have

$$
E_{2}^{\prime}(\theta, \psi) \mid \gamma=\sum_{\alpha=1}^{v} \sum_{\beta=1}^{M} \psi(\alpha) \bar{\theta}(\beta) G_{2}(z ; a \alpha u+c \beta, b \alpha u+d \beta, M)
$$

By (2.5.1), a term in the right-hand side contributes to the constant term only when $a \alpha u+c \beta \equiv 0$ $(M)$, or equivalently, when $c \beta \equiv 0(u)$ and $a \alpha+c \beta / u \equiv 0(v)$. Therefore, the constant term is equal to

$$
\sum_{\substack{\alpha=1 \\ a \alpha u+c \beta \equiv 0(M)}}^{v} \psi(\alpha) \bar{\theta}(\beta)\left(\sum_{m \equiv b \alpha u+d \beta(M)}^{\prime} \frac{1}{m^{2}}\right) .
$$

If $u \nmid c$, then $c \beta \equiv 0(u)$ implies that $(\beta, u) \neq 1$, so that $\bar{\theta}(\beta)=0$ and the above sum vanishes.

We henceforth assume that $u \mid c$. For a given integer $m$, the condition

$$
[a \alpha u+c \beta, b \alpha u+d \beta]=[\alpha u, \beta]\left[\begin{array}{ll}
a & b \\
c & d
\end{array}\right] \equiv[0, m](M)
$$

is equivalent to:

$$
\alpha \equiv-\frac{c}{u} m(v) \quad \text { and } \quad \beta \equiv a m(M)
$$


and these conditions determine $\alpha$ and $\beta$ uniquely in the range considered above. It follows that the constant term is equal to:

$$
\sum_{m}^{\prime} \psi\left(-\frac{c}{u}\right) \psi(m) \bar{\theta}(a) \bar{\theta}(m) \frac{1}{m^{2}}=2 \psi\left(-\frac{c}{u}\right) \bar{\theta}(a) L(2, \psi \bar{\theta}) \prod_{\substack{l \mid M \\ l \nmid \operatorname{cond}(\psi \bar{\theta})}}\left(1-(\psi \bar{\theta})(l) l^{-2}\right)
$$

since $\psi \bar{\theta}$ is even. (Note that $\psi(0)=1$ when $\psi$ is the trivial character.) The functional equation of the $L$-function then implies that this is equal to

$$
2 \psi\left(-\frac{c}{u}\right) \bar{\theta}(a)\left(\prod_{\substack{l \mid M \\ l \nmid \operatorname{cond}(\theta \bar{\psi})}}\left(1-(\psi \bar{\theta})(l) l^{-2}\right)\right) \cdot(-1) \frac{\tau(\psi \bar{\theta})}{2} \frac{4 \pi^{2}}{\operatorname{cond}(\psi \bar{\theta})^{2}} L(1-2, \theta \bar{\psi}),
$$

from which our formula follows.

Later, we will also need the following fact: Let $p$ be a prime not dividing $M$, and denote by $\theta_{p}$ the imprimitive Dirichlet character defined modulo $p u$ induced from $\theta$. We can then consider the Eisenstein series attached to $\theta_{p}$ and $\psi$ defined by the same formula as (2.5.3). We in fact have

$$
E_{2}\left(\theta_{p}, \psi\right)=E_{2}(\theta, \psi)-\theta(p) E_{2}(\theta, \psi) \mid\left[\begin{array}{cc}
p & 0 \\
0 & 1
\end{array}\right]
$$

COROLlary (2.5.7). - Let the notation be as above, and assume that $p \mid c$. Then the constant term of the q-expansion of $E_{2}\left(\theta_{\underline{p}}, \psi\right) \mid \gamma$ is 0 if $u \nmid c$. When $u \mid c$, it is equal to the constant term of $E_{2}(\theta, \psi)$ multiplied by $1-(\theta \bar{\psi})(p) p$.

Proof. - Noting that

$$
E_{2}(\theta, \psi)\left|\left[\begin{array}{cc}
p & 0 \\
0 & 1
\end{array}\right]\left[\begin{array}{ll}
a & b \\
c & d
\end{array}\right]=E_{2}(\theta, \psi)\right|\left[\begin{array}{cc}
a & b p \\
c / p & d
\end{array}\right]\left[\begin{array}{ll}
p & 0 \\
0 & 1
\end{array}\right]
$$

the result follows from (2.5.5) and (2.5.6).

In the above discussions, we excluded the case where $(\theta, \psi)=(\mathbf{1}, \mathbf{1})$. However, as is well known (cf. [3], Math. Werke, p. 474), the series $E_{2}\left(\mathbf{1}_{p}, \mathbf{1}\right)$ also gives the Eisenstein series of weight 2 with respect to $\Gamma_{0}(p)$. Thus when $p \mid c$ (i.e. $\gamma \in \Gamma_{0}(p)$ ), the constant term of $E_{2}\left(\mathbf{1}_{p}, \mathbf{1}\right) \mid \gamma$ is given by $(1-p) \zeta(1-2) / 2$, in accordance with (2.5.7).

\subsection{Proof of (2.4.10)}

We now return to the situation considered in Section 2.4, and keep the same assumption as in (2.4.10). We already know that $\operatorname{Res}(\mathcal{E}(\theta, \psi))$ is contained in $\mathcal{C}_{p}(N)_{\mathfrak{o}}\left[\mathcal{I}^{*}(\theta, \psi)\right]=\Lambda_{\mathfrak{o}}\left(e^{*} \mathbf{e}_{\infty}\right)$ (2.3.6), and want to describe the $\Lambda_{\mathfrak{o}}$-submodule generated by it.

We fix an integer $r \geqslant 1$ for the moment. Setting

$$
\omega_{r}:=(1+T)^{p^{r-1}}-1 \in \Lambda_{\mathfrak{o}}
$$

we recall that the projection induces an isomorphism

$$
\mathcal{C}_{p}(N)_{\mathfrak{o}} / \omega_{r} \stackrel{\sim}{\rightarrow} e^{*} \mathfrak{o}\left[C_{r}\right]
$$


([15], (4.3.14)). Thus the image of $\Lambda_{\mathfrak{o}}\left(e^{*} \mathbf{e}_{\infty}\right)$ in $e^{*} \mathfrak{o}\left[C_{r}\right]$ is the rank one $\Lambda_{\mathfrak{o}} /\left(\omega_{r}\right)$-direct summand $\left(\Lambda_{\mathfrak{o}} /\left(\omega_{r}\right)\right)\left(e^{*} \mathbf{e}_{r}\right)=\mathfrak{o}\left[U_{1} / U_{r}\right]\left(e^{*} \mathbf{e}_{r}\right)$, and we can express the image of $\operatorname{Res}(\mathcal{E}(\theta, \psi))$ in $e^{*} \mathfrak{o}\left[C_{r}\right]$ uniquely in the form

$$
\sum_{\gamma \in U_{1} / U_{r}} c_{r, \gamma} \cdot \iota(\gamma)\left(e^{*} \mathbf{e}_{r}\right) \quad \text { with } c_{r, \gamma} \in \mathfrak{o} .
$$

We are going to compute the coefficients $c_{r, \gamma}$.

Now set

$$
\pi_{\gamma}:=\left(\bigotimes_{j=1}^{k}\left[\begin{array}{c}
1 \\
q_{j}^{h_{j}}
\end{array}\right]_{q_{j}^{e_{j}}}\right) \otimes\left[\begin{array}{l}
0 \\
\gamma
\end{array}\right]_{p^{r}} \quad \bmod \{ \pm 1\} \in C_{r} \text { for } \gamma \in U_{1} / U_{r} .
$$

Then it follows from the definition (2.2.2) of $\mathbf{e}_{r}^{\prime}$ that $\mathbf{e}_{r}$ is a sum of $2 \pi_{1}$ and an o-linear combination of elements of $C_{r}$ different from $\pi_{1}$. Since $e^{*}\left[\begin{array}{c}a \\ c\end{array}\right]_{N_{r}} \equiv\left[\begin{array}{c}a \\ c\end{array}\right]_{N_{r}} \bmod D_{r}^{\prime}$ whenever $p \nmid c$ (2.2.3), we conclude that $2 c_{r, \gamma}$ is equal to the coefficient of the image of $\operatorname{Res}(\mathcal{E}(\theta, \psi))$ with respect to $\pi_{\gamma}$.

In what follows, we put

$$
u^{\prime}:=\prod_{j=1}^{k} q_{j}^{f_{j}}
$$

so that $u=u^{\prime}$ or $u^{\prime} p$, and $N=u^{\prime} v$.

LEMMA (2.6.6). - Take an element $\gamma \in U_{1} / U_{r}\left(\subset\left(\mathbf{Z} / N_{r} \mathbf{Z}\right)^{\times}\right)$, and let $\sigma_{\gamma}$ be an element of $S L_{2}(\mathbf{Z})$ such that $\sigma_{\gamma} \equiv\left[\begin{array}{cc}\gamma^{-1} & * \\ 0 & \gamma\end{array}\right] \bmod N_{r}$. Let $g_{r}$ be defined from $\mathcal{E}(\theta, \psi)$ by the formula (2.4.8). If $\pi_{1}=\left[\begin{array}{l}a \\ c\end{array}\right]_{N_{r}}$ mod $\{ \pm 1\}$ with coprime integers $a$ and $c$, then we have

$$
2 c_{r, \gamma}=\operatorname{Res}_{\pi}\left(\omega_{g_{r} \mid \sigma_{\gamma}^{-1}}\right)
$$

with $\pi:=\left[\begin{array}{c}-c / v \\ a u^{\prime} p^{r}\end{array}\right]_{N_{r}} \bmod \{ \pm 1\}$.

Proof. - We have $\pi_{\gamma}=\sigma_{\gamma} \pi_{1}$. So, by (2.4.9) and the discussion above, we have

$$
2 c_{r, \gamma}=\operatorname{Res}_{\tau_{r}\left(\pi_{\gamma}\right)}\left(\omega_{g_{r}}\right)=\operatorname{Res}_{\tau_{r}\left(\pi_{1}\right)}\left(\omega_{g_{r} \mid \sigma_{\gamma}^{-1}}\right) .
$$

On the other hand, one checks easily that $\tau_{r}\left(\pi_{1}\right)=\pi$.

To calculate $c_{r, \gamma}$ using this, we need the following two elementary facts:

LEMMA (2.6.7). - The "width" of the cusp $\pi$ above is $v$; i.e. if $\delta$ is an element of $S L_{2}(\mathbf{Z})$ such that $\delta(i \infty)=\pi$, then we have

$$
\delta^{-1}\left\{\eta \in \Gamma_{r} \mid \eta(\pi)=\pi\right\} \delta \cdot\{ \pm 1\}=\left\{ \pm\left[\begin{array}{ll}
1 & v \\
0 & 1
\end{array}\right]^{m} \mid m \in \mathbf{Z}\right\} .
$$

Proof. - Straightforward, and left to the reader.

On the other hand, in general, let $\chi_{1}$ and $\chi_{2}$ be Dirichlet characters of conductors $f$ and $g$, respectively. Then it is well known (and easy to see) that:

$$
\tau\left(\chi_{1} \chi_{2}\right)=\chi_{1}(g) \chi_{2}(f) \tau\left(\chi_{1}\right) \tau\left(\chi_{2}\right) \quad \text { if }(f, g)=1 .
$$


Recall that we have set $\xi=\theta \psi^{-1}=\theta \bar{\psi}$ (1.5.3). We are now ready to prove the following

PROPOSITION (2.6.9). - Let the notation be as above, and write $\theta=\theta_{0} \omega^{i}$ with $p$ not dividing the conductor of $\theta_{0}$. Then there is a p-adic unit $C$ contained in a finite cyclotomic extension of $\mathbf{Q}_{p}$ and dependent only on $\theta$ and $\psi$, with which $c_{r, \gamma}$ can be expressed as

$$
\begin{aligned}
C & \times \frac{1}{p^{r-1}} \sum_{\varepsilon \in \widehat{U_{1} / U_{r}}} \varepsilon(\gamma)^{-1} \varepsilon\left(\frac{-N}{\operatorname{cond}\left(\psi \bar{\theta}_{0}\right)}\right) \\
& \times\left(\prod_{\substack{l \mid N \\
l \nmid \operatorname{cond}(\xi)}}\left(1-\left(\xi \omega^{2} \varepsilon\right)^{-1}(l)\langle l\rangle^{-2}\right)\right) G\left(\varepsilon\left(u_{0}\right)-1, \xi \omega^{2}\right)
\end{aligned}
$$

where $G\left(T, \xi \omega^{2}\right)$ is defined in Section 1.4.

Proof. - Using the definition (2.4.8) and the description (1.4.7) of specializations of $\mathcal{E}(\theta, \psi)$, we first note that $g_{r}$ above is given by

$$
\frac{\psi(p)^{-r}}{p^{r-1}} \sum_{\varepsilon \in \widehat{U_{1} / U_{r}}} E_{2}\left((\theta \varepsilon)_{p}, \psi\right)
$$

Here, $(\theta \varepsilon)_{p}$ is the (possibly imprimitive) Dirichlet character modulo $\operatorname{LCM}(\operatorname{cond}(\theta \varepsilon), p)$ induced from $\theta \varepsilon$ (hence $(\theta \varepsilon)_{p}=\theta \varepsilon$ unless $\varepsilon=\mathbf{1}$ ), and $E_{2}\left((\theta \varepsilon)_{p}, \psi\right)$ is defined by the same formula as (2.5.3). By (2.6.6), combined with (2.6.7) and [15], (4.5.4), we see that $v^{-1} 2 c_{r, \gamma}$ is equal to the constant term of the $q$-expansion of

$$
\frac{\psi(p)^{-r}}{p^{r-1}} \sum_{\varepsilon \in \widehat{U_{1} / U_{r}}} \varepsilon(\gamma)^{-1} E_{2}\left((\theta \varepsilon)_{p}, \psi\right)
$$

at the $\operatorname{cusp} \pi$.

But by (2.5.5), (2.5.7) and the remark after it, the constant term of $E_{2}\left((\theta \varepsilon)_{p}, \psi\right)$ at $\pi$ is given by

$$
\begin{aligned}
& \frac{1}{2} \frac{\tau(\psi(\overline{\theta \varepsilon}))}{\tau(\overline{\theta \varepsilon})}\left(\frac{\operatorname{cond}(\overline{\theta \varepsilon})}{\operatorname{cond}(\psi(\overline{\theta \varepsilon}))}\right)^{2} \psi\left(-\frac{a u^{\prime} p^{r}}{\operatorname{cond}(\theta \varepsilon)}\right) \cdot(\overline{\theta \varepsilon})\left(\frac{-c}{v}\right) \\
& \times \prod_{\substack{l \mid \operatorname{cond}(\overline{\theta \varepsilon}) \operatorname{cond}(\psi) \\
l \nmid \operatorname{cond}(\psi(\overline{\theta \varepsilon}))}}\left(1-\psi(\overline{\theta \varepsilon})(l) l^{-2}\right) \times L\left(1-2,(\theta \varepsilon)_{p} \bar{\psi}\right) .
\end{aligned}
$$

If $\operatorname{cond}\left(\omega^{i} \varepsilon\right)=p^{s}$, we see from (2.6.8) above that

$$
\frac{\tau(\psi(\overline{\theta \varepsilon}))}{\tau(\overline{\theta \varepsilon})}=\frac{\tau(\psi(\overline{\theta \varepsilon}))}{\tau\left(\overline{\omega^{i} \varepsilon}\right)} \cdot \frac{\tau\left(\overline{\omega^{i} \varepsilon}\right)}{\tau(\overline{\theta \varepsilon})}=\frac{\tau\left(\psi \bar{\theta}_{0}\right)}{\tau\left(\bar{\theta}_{0}\right)} \psi\left(p^{s}\right) \cdot\left(\omega^{i} \varepsilon\right)\left(\frac{\operatorname{cond}\left(\bar{\theta}_{0}\right)}{\operatorname{cond}\left(\psi \bar{\theta}_{0}\right)}\right) .
$$

On the other hand, it is easy to see that

$$
\left(\frac{\operatorname{cond}(\overline{\theta \varepsilon})}{\operatorname{cond}(\psi(\overline{\theta \varepsilon}))}\right)^{2}=\left(\frac{\operatorname{cond}\left(\bar{\theta}_{0}\right)}{\operatorname{cond}\left(\psi \bar{\theta}_{0}\right)}\right)^{2}
$$




$$
\psi\left(-\frac{a u^{\prime} p^{r}}{\operatorname{cond}(\theta \varepsilon)}\right)=\psi\left(-p^{r-s}\right) \quad \text { and } \quad(\overline{\theta \varepsilon})\left(-\frac{c}{v}\right)=\bar{\theta}_{0}\left(-\frac{c}{v}\right)\left(\omega^{i} \varepsilon\right)(-v) .
$$

It is clear that these four terms are all $p$-adic units in a finite cyclotomic extension of $\mathbf{Q}_{p}$. Also, their product is a product of a $p$-adic unit which depends only on $\theta$ and $\psi$, and

$$
\psi\left(p^{s}\right) \varepsilon\left(\frac{\operatorname{cond}\left(\bar{\theta}_{0}\right)}{\operatorname{cond}\left(\psi \bar{\theta}_{0}\right)}\right) \psi\left(p^{r-s}\right) \varepsilon(-v)=\psi\left(p^{r}\right) \varepsilon\left(-\frac{N}{\operatorname{cond}\left(\psi \bar{\theta}_{0}\right)}\right) .
$$

Now the range of $l$ indicated under the product above is for those $l$ such that $l \mid u^{\prime} v=N$ and $l \nmid \operatorname{cond}(\xi)$. We also have

$$
L\left(1-2,(\theta \varepsilon)_{p} \bar{\psi}\right)=G\left(\varepsilon\left(u_{0}\right)-1, \xi \omega^{2}\right) .
$$

Thus combining what we have said, the proposition follows.

We now prove (2.4.10): If $\operatorname{Res}(\mathcal{E}(\theta, \psi))=F_{0}(T)\left(e^{*} \mathbf{e}_{\infty}\right)$ with $F_{0}(T) \in \Lambda_{\mathfrak{o}}$, then its image in $\left(\Lambda_{\mathfrak{o}} /\left(\omega_{r}\right)\right)\left(e^{*} \mathbf{e}_{r}\right)=\mathfrak{o}\left[U_{1} / U_{r}\right]\left(e^{*} \mathbf{e}_{r}\right)$ is given by

$$
\sum_{\gamma \in U_{1} / U_{r}}\left(\frac{1}{p^{r-1}} \sum_{\varepsilon \in \widehat{U_{1} / U_{r}}} \varepsilon(\gamma)^{-1} F_{0}\left(\varepsilon\left(u_{0}\right)-1\right)\right) \cdot \iota(\gamma)\left(e^{*} \mathbf{e}_{r}\right)
$$

for all $r \geqslant 1$ (cf. e.g. the proof of [14], (2.4.2)). Conversely, this property determines $F_{0}(T)$ uniquely.

Define $\alpha \in \mathbf{Z}_{p}$ by

$$
\left\langle-\frac{N}{\operatorname{cond}\left(\psi \bar{\theta}_{0}\right)}\right\rangle=u_{0}^{\alpha}
$$

so that the value of $(1+T)^{\alpha} \in \Lambda_{\mathfrak{o}}^{\times}$at $T=\varepsilon\left(u_{0}\right)-1$ is $\varepsilon\left(-N / \operatorname{cond}\left(\psi \bar{\theta}_{0}\right)\right)$. If we set

$$
F(T):=C(1+T)^{\alpha}\left(\prod_{\substack{l \mid N \\ l \nmid \operatorname{cond}(\xi)}}\left(1-\left(\xi \omega^{2}\right)^{-1}(l)\langle l\rangle^{-2}(1+T)^{-s(l)}\right)\right) G\left(T, \xi \omega^{2}\right) \in \Lambda_{\mathfrak{o}}
$$

then it follows from (2.6.9) that this $F(T)$ enjoys the characterizing property of $F_{0}(T)$, and we conclude that $F_{0}(T)=F(T)$. Clearly, $F(T)$ is a multiple of $A(T ; \theta, \psi)$ by a unit in $\Lambda_{\mathfrak{o}}$; and the proof of (2.4.10) is complete.

\section{Determination of congruence modules of Eisenstein type}

\subsection{Congruence modules for $\Lambda$-adic modular forms}

The purpose of this section is to prove our main theorem (1.5.5). We treat the case (I) in this subsection.

We consider the same situation as in Section 1.5: Let $\theta, \psi, u, v$, and $\mathfrak{r}$ be as stated at the beginning of Section 1.4, with $u v=N$ or $N p$, and assume that $p \nmid \varphi(N)$. Recall that $\mathfrak{M}(\theta, \psi)=: \mathfrak{M}$ (resp. $\mathfrak{M}^{*}(\theta, \psi)=: \mathfrak{M}^{*}$ ) is the Eisenstein maximal ideal of $e \mathcal{H}(N ; \mathfrak{r})$ (resp. $\left.e^{*} \mathcal{H}^{*}(N ; \mathfrak{r})\right)$ associated with $\mathcal{E}(\theta, \psi)$. 
LEMMA (3.1.1). $-(\mathbf{Z} / N p \mathbf{Z})^{\times}$acts on the localization $\mathcal{H}(N ; \mathfrak{r})_{\mathfrak{M}}$ through the character $\theta \psi$, and $T(q, q)=(\theta \psi)(q) \iota(\langle q\rangle)$ in $\mathrm{eH}(N ; \mathfrak{r})_{\mathfrak{M}}$ for all positive integers $q$ prime to $N p$. The same holds for $e^{*} \mathcal{H}^{*}(N ; \mathfrak{r})_{\mathfrak{M}^{*}}$.

Proof. - It is enough to prove the assertion for $e \mathcal{H}(N ; \mathfrak{r})_{\mathfrak{M}}$, and we may assume that $\mathfrak{r}$ contains all the $\varphi(N p)$ th roots of unity to do this. Then, since $p \nmid \varphi(N)$, the action of $(\mathbf{Z} / N p \mathbf{Z})^{\times}$on the local ring $e \mathcal{H}(N ; \mathfrak{r})_{\mathfrak{M}}$ must factor through a character, and by (1.4.8), this character can be nothing other than $\theta \psi$. The assertion for $T(q, q)$ also follows from this.

Proposition (3.1.2). $-\mathcal{C}_{p}(N)_{\mathfrak{r}, \mathfrak{M}^{*}}$ is a free $\Lambda_{\mathfrak{r}}$-module of rank one when $(\theta, \psi)$ is not exceptional (1.4.10). If $(\theta, \psi)$ is exceptional, it is free of rank two over $\Lambda_{\mathfrak{r}}$.

Proof. - We may again assume that $\mathfrak{r}$ contains all the $\varphi(N p)$ th roots of unity.

$\mathcal{C}_{p}(N)_{\mathfrak{r}, \mathfrak{M}^{*}}$, being a direct factor of a free $\Lambda_{\mathfrak{r}}$-module, is $\Lambda_{\mathfrak{r}}$-free, and its rank is positive by (2.3.6). Assume that the rank is strictly greater than one. Then we see from the exact sequence (2.4.6) that there would exist a non-zero element $x \in \mathcal{C}_{p}(N)_{\mathfrak{r}, \mathfrak{M}^{*}}$ for which the eigenvalues of $T^{*}(l)$ coincide with the eigenvalues of $T(l)$ for $\mathcal{E}\left(\theta^{\prime}, \psi^{\prime} ; c\right) \in e M\left(N ; \Lambda_{\mathfrak{r}}\right)$ with some $\left(\theta^{\prime}, \psi^{\prime}\right) \neq(\theta, \psi)$, for all primes $l \nmid N p$. By (1.4.9), (i), we must have: $\left(\theta^{\prime}, \psi^{\prime}\right)=\left(\psi \omega^{-1}, \theta \omega\right)$. Consequently, since $u v=N$ or $N p$, we have $c=1$, and $x$ shares the same eigenvalues for all prime numbers $l$ with $\mathcal{E}\left(\psi \omega^{-1}, \theta \omega\right)$, in the same sense as above. When $(\theta, \psi)$ is not exceptional, we get a contradiction by (1.4.9), (ii). In the exceptional case, the same lemma implies that the $\Lambda_{\mathfrak{r}}$-rank of $\mathcal{C}_{p}(N)_{\mathfrak{r}, \mathfrak{M}^{*}}$ is two.

From now on, we assume that $(\theta, \psi)$ is non-exceptional, and $K$ is a complete subfield of $\mathbf{C}_{p}$ containing $k$ and all the roots of unity, in this subsection. By the above proposition and (2.3.6), we have:

$$
\mathcal{C}_{p}(N)_{\mathfrak{r}, \mathfrak{M}^{*}} \otimes_{\Lambda_{\mathfrak{r}}} \Lambda_{\mathfrak{o}} \cong \mathcal{C}_{p}(N)_{\mathfrak{o}, \mathfrak{M}^{*}}=\Lambda_{\mathfrak{o}}\left(e^{*} \mathbf{e}_{\infty}\right) .
$$

Now consider (2.4.6) as an exact sequence of $e \mathcal{H}(N ; \mathfrak{r})$-modules, viewing $\mathcal{C}_{p}(N)_{\mathfrak{o}}$ as a module over this ring through the canonical isomorphism: $e \mathcal{H}(N ; \mathfrak{r}) \stackrel{\sim}{\rightarrow} e^{*} \mathcal{H}^{*}(N ; \mathfrak{r})$. Then, its localization at $\mathfrak{M}$ becomes

$$
0 \rightarrow e S\left(N ; \Lambda_{\mathfrak{o}}\right)_{\mathfrak{M}} \rightarrow e M\left(N ; \Lambda_{\mathfrak{o}}\right)_{\mathfrak{M}} \stackrel{\mathbf{R e s}}{\longrightarrow} \Lambda_{\mathfrak{o}}\left(e^{*} \mathbf{e}_{\infty}\right) \rightarrow 0
$$

Noting that $e M\left(N ; \Lambda_{\mathfrak{r}}\right) \otimes_{\Lambda_{\mathfrak{r}}} \Lambda_{\mathfrak{o}} \cong e M\left(N ; \Lambda_{\mathfrak{o}}\right)$ canonically (and similarly for cusp forms), and using the faithful flatness of $\Lambda_{\mathfrak{o}}$ over $\Lambda_{\mathfrak{r}}$ (cf. [16], (2.1.1)), we see that $e M\left(N ; \Lambda_{\mathfrak{r}}\right)_{\mathfrak{M}} / e S\left(N ; \Lambda_{\mathfrak{r}}\right)_{\mathfrak{M}}$ is $\Lambda_{\mathfrak{r}}$-free of rank one, and we have the exact sequence of $e \mathcal{H}(N ; \mathfrak{r})_{\mathfrak{M}}$-modules

$$
0 \rightarrow e S\left(N ; \Lambda_{\mathfrak{r}}\right)_{\mathfrak{M}} \stackrel{i}{\rightarrow} e M\left(N ; \Lambda_{\mathfrak{r}}\right)_{\mathfrak{M}} \stackrel{\pi}{\rightarrow} \Lambda_{\mathfrak{r}} \rightarrow 0
$$

where $e \mathcal{H}(N ; \mathfrak{r})_{\mathfrak{M}}$ acts on $\Lambda_{\mathfrak{r}}$ through its quotient $e \mathcal{H}(N ; \mathfrak{r})_{\mathfrak{M}} / \mathcal{I}(\theta, \psi)_{\mathfrak{M}}=\Lambda_{\mathfrak{r}}$. This is the exact sequence stated in (1.5.5), (I). It splits uniquely as modules over the Hecke algebra when tensored with $\mathcal{L}_{k}$ (the quotient field of $\Lambda_{\mathfrak{r}}$ ) over $\Lambda_{\mathfrak{r}}$. When $(\theta, \psi)=\left(\omega^{-2}, \mathbf{1}\right)$, the mapping $\pi$ may be identified with the one taking a $\Lambda$-adic modular form to its constant term of the formal $q$-expansion, and the congruence module vanishes in this case, from the argument of [15], 5.2. We assume that $(\theta, \psi) \neq\left(\omega^{-2}, \mathbf{1}\right)$ in the following.

By (2.4.10) and its proof, when $\mathfrak{r}$ contains the constant $C$ in (2.6.9), the residue mapping sends $e M\left(N ; \Lambda_{\mathfrak{r}}\right)_{\mathfrak{M}}$ to $\Lambda_{\mathfrak{r}}\left(e^{*} \mathbf{e}_{\infty}\right)$, and (3.1.5) may be identified with

$$
0 \rightarrow e S\left(N ; \Lambda_{\mathfrak{r}}\right)_{\mathfrak{M}} \stackrel{i}{\rightarrow} e M\left(N ; \Lambda_{\mathfrak{r}}\right)_{\mathfrak{M}} \stackrel{\text { Res }}{\rightarrow} \Lambda_{\mathfrak{r}}\left(e^{*} \mathbf{e}_{\infty}\right) \rightarrow 0 .
$$


(See Section 3.3 below for another description of Coker( $i$ ).) To prove (1.5.5) for (I), we may assume that we are in this case (or we may even replace $\mathfrak{r}$ by $\mathfrak{o}$ ), by (1.1.7) and (1.1.9).

Now if we denote by $s$ the section of $\operatorname{Res} \otimes_{\Lambda_{\mathfrak{r}}} 1_{\mathcal{L}_{k}}$, its image is the $\mathcal{L}_{k}$-vector subspace spanned by $\mathcal{E}(\theta, \psi)$. By (2.4.10), we in fact have

$$
s\left(e^{*} \mathbf{e}_{\infty}\right)=U A(T ; \theta, \psi)^{-1} \mathcal{E}(\theta, \psi)
$$

with a unit $U$ in $\Lambda_{\mathfrak{r}}$. Since the coefficient of $q^{p}$ in $\mathcal{E}(\theta, \psi)$ is $\psi(p) \in \mathfrak{r}^{\times}$, we conclude that

$$
\boldsymbol{\operatorname { R e s }}\left(e M\left(N ; \Lambda_{\mathfrak{r}}\right)_{\mathfrak{M}} \cap s\left(\Lambda_{\mathfrak{r}}\left(e^{*} \mathbf{e}_{\infty}\right)\right)\right)=A(T ; \theta, \psi) \cdot \Lambda_{\mathfrak{r}}\left(e^{*} \mathbf{e}_{\infty}\right)
$$

which completes the proof of the part (I) of (1.5.5).

As a consequence, we obtain the following result. When $\psi=1$, it had been proved by Wiles ([22], Theorem 4.1).

Proposition (3.1.9). - The notation being as in (1.5.1), there is a surjective $\Lambda_{\mathfrak{r}}$-algebra homomorphism

$$
e h(N ; \mathfrak{r})_{\mathfrak{M}} / I(\theta, \psi)_{\mathfrak{M}} \rightarrow \Lambda_{\mathfrak{r}} /(A(T ; \theta, \psi)) .
$$

Proof. - Considering the congruence module attached to (3.1.5) with the canonical splitting as

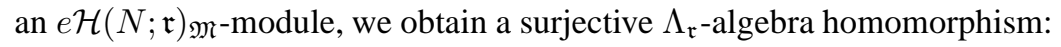

$$
e \mathcal{H}(N ; \mathfrak{r})_{\mathfrak{M}} \rightarrow \Lambda_{\mathfrak{r}} /(A(T ; \theta, \psi))
$$

In view of the description as " $t(B) / A$ " of the congruence module in (1.1.4), this must factor through $e \mathcal{H}(N ; \mathfrak{r})_{\mathfrak{M}} \rightarrow e h(N ; \mathfrak{r})_{\mathfrak{M}}$, while the description as " $C / \pi(B \cap s(C))$ " loc. cit. implies that this further factors through $e h(N ; \mathfrak{r})_{\mathfrak{M}} / I(\theta, \psi)_{\mathfrak{M}}$.

Corollary (3.1.10). - If $A(T ; \theta, \psi)$ is not a unit in $\Lambda_{\mathfrak{r}}$, then $I(\theta, \psi)$, and hence $\mathfrak{m}(\theta, \psi)$ also, is a proper ideal of $e h(N ; \mathfrak{r})$.

\subsection{Congruence modules for Hecke algebras}

For the moment, we give some general remarks under the setting as in Section 1.1. We thus consider the exact sequence (1.1.1) of flat $R$-modules, and its splitting (1.1.2) over $L$.

Assume that we are given an $R$-subalgebra $H$ of $\operatorname{End}_{R}(B)$, which is commutative, unitary and $R$-flat. We also assume that (1.1.1) is an exact sequence of $H$-modules, and that (1.1.2) is a splitting of $H \otimes_{R} L$-modules. Then $H$ naturally embeds in $\operatorname{End}_{R}\left(A \otimes_{R} L\right) \oplus \operatorname{End}_{R}\left(C \otimes_{R} L\right)$. Let $\mathrm{pr}_{1}$ (resp. $\mathrm{pr}_{2}$ ) be the projection mapping from $H$ to the first (resp. the second) direct factor, and set $H_{i}:=\operatorname{pr}_{i}(H)$ for $i=1,2$. Define ideals $\mathfrak{a}$ and $\mathfrak{b}$ of $H$ by the exactness of the following sequences:

$$
\left\{\begin{array}{l}
0 \rightarrow \mathfrak{a} \rightarrow H \stackrel{\mathrm{pr}_{1}}{\rightarrow} H_{1} \rightarrow 0, \\
0 \rightarrow \mathfrak{b} \rightarrow H \stackrel{\mathrm{pr}_{2}}{\rightarrow} H_{2} \rightarrow 0 .
\end{array}\right.
$$

Assume furthermore that $H_{1}$ and $H_{2}$ are both $R$-flat (from which the $R$-flatness of $\mathfrak{a}$ and $\mathfrak{b}$ follows), and that $H \otimes_{R} L \stackrel{\stackrel{\sim}{\rightarrow}}{(}\left(H_{1} \otimes_{R} L\right) \oplus\left(H_{2} \otimes_{R} L\right)$ via $\mathrm{pr}_{1}$ and $\mathrm{pr}_{2}$. From the last condition, we obtain canonical splittings of the two sequences above when tensored with $L$ over $R$, and we can consider the associated congruence modules. These are both isomorphic to

$$
\left(H_{1} \oplus H_{2}\right) / H=: \mathcal{C} .
$$


On the other hand, it is clear that $\mathfrak{b}=\operatorname{Ann}_{H}(C)$, the annihilator of $C$ in $H$, and this is mapped injectively into $H_{1}$ via $\mathrm{pr}_{1}$. Thus (1.1.4) gives the following description:

$$
\mathcal{C} \cong H_{1} / \operatorname{pr}_{1}\left(\operatorname{Ann}_{H}(C)\right)
$$

We now come back to the situation studied in Section 3.1. We assume that $(\theta, \psi)$ is nonexceptional, and apply the argument above to the exact sequence (3.1.5) with $R=\Lambda_{\mathfrak{r}}$ and $H=e \mathcal{H}(N ; \mathfrak{r})_{\mathfrak{M}}$. Clearly, we have $H_{1}=e h(N ; \mathfrak{r})_{\mathfrak{M}}$ and $H_{2}=\Lambda_{\mathfrak{r}}$. Noting that $\mathrm{pr}_{2}$ in the present case cannot factor through $H_{1}$, we see that all the conditions above are satisfied. Also, from definition (1.5.1), $\mathfrak{b}=\mathcal{I}(\theta, \psi)_{\mathfrak{M}}$ and $\operatorname{pr}_{1}(\mathfrak{b})=I(\theta, \psi)_{\mathfrak{M}}$, so that the second exact sequence in (3.2.1) is nothing but the one labelled as (II) in (1.5.5):

$$
0 \rightarrow \mathcal{I}(\theta, \psi)_{\mathfrak{M}} \rightarrow e \mathcal{H}(N ; \mathfrak{r})_{\mathfrak{M}} \rightarrow \Lambda_{\mathfrak{r}} \rightarrow 0
$$

By (3.2.3), the associated congruence module is isomorphic to the cuspidal Hecke algebra modulo Eisenstein ideal: $e h(N ; \mathfrak{r})_{\mathfrak{M}} / I(\theta, \psi)_{\mathfrak{M}}$, and we want to show that this is isomorphic to $\Lambda_{\mathfrak{r}} /(A(T ; \theta, \psi))$ when $(\theta, \psi) \neq\left(\omega^{-2}, \mathbf{1}\right)$, and $\{0\}$ otherwise. In the former case, (3.1.9) assures us that the two modules above vanish when $I(\theta, \psi)_{\mathfrak{M}}=e h(N ; \mathfrak{r})_{\mathfrak{M}}$; and we are reduced to the case where $\mathfrak{m}(\theta, \psi)$ is a proper ideal. Then, when $\psi=\mathbf{1}$ and $\left.\theta\right|_{(\mathbf{Z} / p \mathbf{Z}) \times}=\omega^{i}$ with $i \not \equiv 0,-1$ mod $(p-1)$, the comparison of the two modules above was done in [15], (5.3.21) through an argument involving the Iwasawa main conjecture for $\mathbf{Q}$ (a theorem of Mazur and Wiles [11]). Even when $\theta=\omega^{-2}$, or $i \equiv 0$ or -1 , a similar argument applies after [16] (with the aid of results in Section 3.4 below). Since this task is totally routine, we postpone it until we treat the Galois representation theoretical aspect of our theory for more general $(\theta, \psi)$ in the appendix, where the method of Harder and Pink [2], and Kurihara [9] is employed. Here, we prove (1.5.5) in the case (II) under the assumption that $\psi$ is not the trivial character.

For this, we recall the duality between $\Lambda$-adic modular forms and $p$-adic Hecke algebras: We set

$$
e m\left(N ; \Lambda_{\mathfrak{o}}\right):=\left(e M\left(N ; \Lambda_{\mathfrak{o}}\right) \otimes_{\Lambda_{\mathfrak{o}}} \mathcal{L}_{K}\right) \cap\left(\mathcal{L}_{K}+q \Lambda_{\mathfrak{o}} \llbracket q \rrbracket\right)
$$

for any $K$ and $\mathfrak{o}$ as before (which may not contain all the roots of unity). Then, we know that this is a free $\Lambda_{\mathfrak{o}}$-module of finite rank, and stable under the action of $e \mathcal{H}(N ; \mathfrak{o})$ on $e M\left(N ; \Lambda_{\mathfrak{o}}\right) \otimes_{\Lambda_{\mathfrak{o}}} \mathcal{L}_{K}$. Define pairings

$$
\left\{\begin{array}{l}
e h(N ; \mathfrak{o}) \times e S\left(N ; \Lambda_{\mathfrak{o}}\right) \rightarrow \Lambda_{\mathfrak{o}} \\
\operatorname{eH}(N ; \mathfrak{o}) \times e m\left(N ; \Lambda_{\mathfrak{o}}\right) \rightarrow \Lambda_{\mathfrak{o}}
\end{array}\right.
$$

by the formula $(t, \mathcal{F}):=($ the coefficient of $q$ in the formal $q$-expansion of $\mathcal{F} \mid t)$. Then we also know that these pairings establish perfect dualities of free $\Lambda_{\mathfrak{o}}$-modules (Hida [7], 7.3; cf. also [14], (2.5.3) and [15], (2.5.5)).

Lemma (3.2.7). - Assume that $\psi \neq 1$ and $(\theta, \psi)$ is not exceptional. For $K$ containing $k$, the localizations em $\left(N ; \Lambda_{\mathfrak{o}}\right)_{\mathfrak{M}}$ and $\operatorname{eM}\left(N ; \Lambda_{\mathfrak{o}}\right)_{\mathfrak{M}}$ at $\mathfrak{M}=\mathfrak{M}(\theta, \psi)(\subset e \mathcal{H}(N ; \mathfrak{r}))$ coincide.

Proof. - It is clear that $e M\left(N ; \Lambda_{\mathfrak{o}}\right)_{\mathfrak{M}} \subseteq e m\left(N ; \Lambda_{\mathfrak{o}}\right)_{\mathfrak{M}}$, and that these become the same when tensored with $\mathcal{L}_{K}$ over $\Lambda_{\mathfrak{o}}$. On the other hand, we already know that $e M\left(N ; \Lambda_{\mathfrak{o}}\right)_{\mathfrak{M}} \otimes_{\Lambda_{\mathfrak{o}}} \mathcal{L}_{K}$ is spanned by $e S\left(N ; \Lambda_{\mathfrak{o}}\right)_{\mathfrak{M}} \otimes_{\Lambda_{\mathfrak{o}}} \mathcal{L}_{K}$ and $\mathcal{E}(\theta, \psi)$ over $\mathcal{L}_{K}$. Since $\psi \neq \mathbf{1}$, the constant term of the formal $q$-expansion of $\mathcal{E}(\theta, \psi)$ vanishes, and our claim reduces to the equality: $\left(e M\left(N ; \Lambda_{\mathfrak{o}}\right) \otimes_{\Lambda_{\mathfrak{o}}}\right.$ $\left.\mathcal{L}_{K}\right) \cap \Lambda_{\mathfrak{o}} \llbracket q \rrbracket=e M\left(N ; \Lambda_{\mathfrak{o}}\right)$. This follows from [15], (2.4.9) and the final equality in the proof of [15], (2.4.7); but see also the corrections to [15] at the end of this paper for the latter. 
Let us now consider the $\Lambda_{\mathfrak{r}}$-dual of the exact sequence (3.1.5). Since the pairings above satisfy $\left(t^{\prime} t, \mathcal{F}\right)=\left(t, \mathcal{F} \mid t^{\prime}\right)$, this reads

$$
0 \rightarrow \Lambda_{\mathfrak{r}} \rightarrow e \mathcal{H}(N ; \mathfrak{r})_{\mathfrak{M}} \stackrel{\pi}{\rightarrow} e h(N ; \mathfrak{r})_{\mathfrak{M}} \rightarrow 0
$$

where $\pi$ is the obvious surjection. Namely, this is exactly the first exact sequence in (3.2.1) in the present situation. Thus the congruence module attached to this sequence together with natural splitting is isomorphic to that for (3.2.4) which is generated by one element as a $\Lambda_{\mathfrak{r}}$-module by (1.1.9). We conclude from (1.1.12) that the congruence module in question is isomorphic to the one in the case (I). This, with the remainder (in the case where $\psi=1$ ) treated in the appendix in greater generality, settles the case (II) of (1.5.5).

Remark (3.2.9). - In [4], at the end of the paper, Hida conjectured that the congruence module attached to $\mathcal{E}(\theta, \psi)$ is described in terms of Kubota-Leopoldt $p$-adic $L$-function. Precisely, such a $\Lambda$-adic Eisenstein series determines a direct summand $\mathcal{K}$ of $e \mathcal{H}(N ; \mathfrak{r}) \otimes_{\Lambda_{\mathfrak{r}}} \mathcal{L}_{k}$, which is isomorphic to $\mathcal{L}_{k}$. (If $\theta=\chi \omega^{a-2}$ with $\chi$ a Dirichlet character of prime-to- $p$ conductor, this is the irreducible component attached by Hida, to $\chi, \psi$ and $a$, in [4], Theorem 7.2.) Since $e h(N ; \mathfrak{r})_{\mathfrak{M}}$ is free over $\Lambda_{\mathfrak{r}}$, the congruence module above coincides with the one defined in [4], (3.9 a) for $\mathcal{K}$. Thus our result gives an affirmative answer to his conjecture under the assumption that $p \nmid \varphi(N)$ and $(\theta, \psi)$ is not exceptional.

\subsection{Remark on the duality (3.2.6)}

Here, we would like to complement (3.2.7) by the following

Proposition (3.3.1). - Let the notation and the assumption be as stated at the beginning of Section 3.1. If either $\psi \neq \mathbf{1}$ and $\theta \omega \neq \mathbf{1}$; or $(\theta, \psi)$ is different from $\left(\omega^{-2}, \mathbf{1}\right)$ and nonexceptional, then we have em $\left(N ; \Lambda_{\mathfrak{o}}\right)_{\mathfrak{M}}=e M\left(N ; \Lambda_{\mathfrak{o}}\right)_{\mathfrak{M}}$ for $\mathfrak{M}=\mathfrak{M}(\theta, \psi)$. Consequently, the second pairing in (3.2.6) induces a perfect duality between the free $\Lambda_{\mathfrak{o}}$-modules $\mathrm{eH}(N ; \mathfrak{o})_{\mathfrak{M}}$ and $e M\left(N ; \Lambda_{\mathfrak{o}}\right)_{\mathfrak{M}}$.

Proof. - When $(\theta, \psi)$ is exceptional, eM(N; $\left.\Lambda_{\mathfrak{o}}\right)_{\mathfrak{M}} \otimes_{\Lambda_{\mathfrak{o}}} \mathcal{L}_{K}$ is generated over $\mathcal{L}_{K}$ by $e S\left(N ; \Lambda_{\mathfrak{o}}\right)_{\mathfrak{M}}, \mathcal{E}(\theta, \psi)$ and $\mathcal{E}\left(\psi \omega^{-1}, \theta \omega\right)$. If $\psi$ and $\theta \omega$ are non-trivial, the constant terms of the $q$-expansions of these two $\Lambda$-adic Eisenstein series vanish, and the same reasoning as (3.2.7) applies to conclude.

So, let us assume that $(\theta, \psi)$ is not exceptional, but $\psi=\mathbf{1}$ and $\theta \neq \omega^{-2}$. We first note that we may replace $\mathfrak{o}$ by $\mathfrak{r}$ which is finite over $\mathbf{Z}_{p}$. In fact, if our assertion is established for $\mathfrak{r}$, then the resulting duality, after tensoring $\Lambda_{\mathfrak{o}}$ over $\Lambda_{\mathfrak{r}}$, yields an isomorphism

$$
e M\left(N ; \Lambda_{\mathfrak{o}}\right)_{\mathfrak{M}} \stackrel{\sim}{\rightarrow} \operatorname{Hom}_{\Lambda_{\mathfrak{o}}}\left(e \mathcal{H}(N ; \mathfrak{o})_{\mathfrak{M}}, \Lambda_{\mathfrak{o}}\right)
$$

and hence the duality (3.2.6) implies that $e m\left(N ; \Lambda_{\mathfrak{o}}\right)_{\mathfrak{M}}=e M\left(N ; \Lambda_{\mathfrak{o}}\right)_{\mathfrak{M}}$.

Now in this case, we use the isomorphism

$$
e h(N ; \mathfrak{r})_{\mathfrak{M}} / I(\theta, \mathbf{1})_{\mathfrak{M}} \cong \Lambda_{\mathfrak{r}} /\left(G\left(T, \theta \omega^{2}\right)\right)
$$

established in the appendix, to prove our assertion. We take an element $\mathcal{F} \in \operatorname{em}\left(N ; \Lambda_{\mathfrak{r}}\right)_{\mathfrak{M}}$ and express it as

$$
\mathcal{F}=b(T) \mathcal{E}(\theta, \mathbf{1})+c(T) \mathcal{F}_{\text {cusp }}
$$

with $b(T), c(T) \in \mathcal{L}_{k}$ and $\mathcal{F}_{\text {cusp }} \in e S\left(N ; \Lambda_{\mathfrak{r}}\right)_{\mathfrak{M}}$. Write $b(T)$ as $b_{\mathrm{n}}(T) / b_{\mathrm{d}}(T)$ with mutually coprime $b_{\mathrm{n}}(T), b_{\mathrm{d}}(T) \in \Lambda_{\mathfrak{r}}$. What we want to show is that $b_{\mathrm{d}}(T) \mid G\left(T, \theta \omega^{2}\right)$. 
For this, put $\mathcal{F}_{0}:=b_{\mathrm{d}}(T) c(T) \mathcal{F}_{\text {cusp }}=b_{\mathrm{d}}(T) \mathcal{F}-b_{\mathrm{n}}(T) \mathcal{E}(\theta, \mathbf{1})$. This belongs to

$$
\left(e S\left(N ; \Lambda_{\mathfrak{r}}\right)_{\mathfrak{M}} \otimes_{\Lambda_{\mathfrak{r}}} \mathcal{L}_{k}\right) \cap \Lambda_{\mathfrak{r}} \llbracket q \rrbracket=e S\left(N ; \Lambda_{\mathfrak{r}}\right)_{\mathfrak{M}}
$$

It is then easy to see that

$$
\mathcal{F}_{0} \mid T(l) \equiv\left(\theta(l) A_{l}(T)+1\right) \mathcal{F}_{0} \bmod b_{\mathrm{d}}(T) \Lambda_{\mathfrak{r}} \llbracket q \rrbracket
$$

if $l$ is a prime number different from $p$, and $\mathcal{F}_{0} \mid T(p) \equiv \mathcal{F}_{0} \bmod b_{\mathrm{d}}(T) \Lambda_{\mathfrak{r}} \llbracket q \rrbracket$; i.e. $\mathcal{F}_{0}$ is transformed under $T(l)$ 's in the same manner as $\mathcal{E}(\theta, \mathbf{1})$ modulo $b_{\mathrm{d}}(T) \Lambda_{\mathrm{r}} \llbracket q \rrbracket$.

But since the coefficient of $q^{p}$ in $\mathcal{F}_{0}$ is prime to $b_{\mathrm{d}}(T)$, we see that the image of $\mathcal{F}_{0}$ in $e S\left(N ; \Lambda_{\mathfrak{r}}\right)_{\mathfrak{M}} / b_{\mathrm{d}}(T) \hookrightarrow\left(\Lambda_{\mathfrak{r}} /\left(b_{\mathrm{d}}(T)\right)\right) \llbracket q \rrbracket$ spans a free $\Lambda_{\mathfrak{r}} /\left(b_{\mathrm{d}}(T)\right)$-module of rank one. The action on this module gives us a $\Lambda_{\mathfrak{r}}$-algebra homomorphism: $e h(N ; \mathfrak{r})_{\mathfrak{M}} \rightarrow \Lambda_{\mathfrak{r}} /\left(b_{\mathrm{d}}(T)\right)$ sending each $T(l)$ to the eigenvalue of $T(l)$ for $\mathcal{E}(\theta, \mathbf{1})$, which must factor through $e h(N ; \mathfrak{r})_{\mathfrak{M}} / I(\theta, \mathbf{1})_{\mathfrak{M}}$. From the above mentioned isomorphism, we conclude that $\left(G\left(T, \theta \omega^{2}\right)\right) \subseteq\left(b_{\mathrm{d}}(T)\right)$.

Thus, for non-exceptional $(\theta, \psi) \neq\left(\omega^{-2}, \mathbf{1}\right)$ with $\psi=\mathbf{1}$, we have used our main result (1.5.5) in the case (II) to prove (3.3.1). As the argument in the previous subsection shows, if one could prove (3.3.1) directly somehow, it would conversely give a proof of (1.5.5) for such $(\theta, \psi)$ in the case (II).

We now give another description of $\operatorname{Ker}(\pi)$ (resp. $\operatorname{Coker}(i)$ ) in the sequence (3.2.8) (resp. (3.1.6)). This was suggested by the anonymous referee to whom the author is thankful. It is based on the exact sequence due to Hida [7], Theorem 2.2:

$$
0 \rightarrow \bar{S}(N ; \mathfrak{r}) \rightarrow \overline{\mathcal{M}}(N ; \mathfrak{r}) \rightarrow C\left(S \times \mathbf{Z}_{p}^{\times} ; \mathfrak{r}\right) \rightarrow 0
$$

assuming that $\mathfrak{r}$ contains primitive $N$ th roots of unity. Here, $\bar{S}(N ; \mathfrak{r})($ resp. $\overline{\mathcal{M}}(N ; \mathfrak{r}))$ is the space of $p$-adic cusp forms (resp. modular forms) of level $N$ over $\mathfrak{r}$ as defined in [7], §1, and $C\left(S \times \mathbf{Z}_{p}^{\times} ; \mathfrak{r}\right)$ is the space of $\mathfrak{r}$-valued continuous functions on $S \times \mathbf{Z}_{p}^{\times}$(the projective limit of the set of unramified cusps of the tower $\left.\left\{X_{1}\left(N p^{r}\right)\right\}_{r \geqslant 0}\right)$.

Now applying the idempotent $e$, localizing at $\mathfrak{M}$, and then taking the $\mathfrak{r}$-dual, we obtain an exact sequence

$$
0 \rightarrow \operatorname{Meas}\left(S \times \mathbf{Z}_{p}^{\times} ; \mathfrak{r}\right)_{\mathfrak{M}} \rightarrow e \mathcal{H}(N ; \mathfrak{r})_{\mathfrak{M}} \stackrel{\pi}{\rightarrow} e h(N ; \mathfrak{r})_{\mathfrak{M}} \rightarrow 0
$$

when $(\theta, \psi) \neq\left(\omega^{-2}, \mathbf{1}\right)$. Here, the module in the left-hand side is the one consisting of $\mathfrak{r}$-valued measures on $S \times \mathbf{Z}_{p}^{\times}$. Indeed, Hida proved the (r-linear) duality between $\bar{S}(N ; \mathfrak{r})$ and $h(N ; \mathfrak{r})$ ([7], Theorem 1.3), and one can proceed in a similar manner (but taking account of a subtle difference between the $\mathfrak{r}$-dual of $\overline{\mathcal{M}}(N ; \mathfrak{r})$ and $\mathcal{H}(N ; \mathfrak{r}))$ to obtain the duality for modular forms under the assumption on characters above. Note that this especially shows the $\Lambda_{\mathfrak{r}}$-freeness of $\operatorname{Ker}(\pi)$.

Next, taking the $\Lambda_{\mathfrak{r}}$-dual of (3.3.3), we also obtain an exact sequence

$$
0 \rightarrow e S\left(N ; \Lambda_{\mathfrak{r}}\right)_{\mathfrak{M}} \stackrel{i}{\rightarrow} e M\left(N ; \Lambda_{\mathfrak{r}}\right)_{\mathfrak{M}} \rightarrow \operatorname{Hom}_{\Lambda_{\mathfrak{r}}}\left(\operatorname{Meas}\left(S \times \mathbf{Z}_{p}^{\times} ; \mathfrak{r}\right)_{\mathfrak{M}}, \Lambda_{\mathfrak{r}}\right) \rightarrow 0
$$

under the same hypothesis as in (3.3.1).

It is tempting to treat the case (II) of (1.5.5) directly through (3.3.2) and (3.3.3), but the author has not worked it out. 


\subsection{Galois action on $p$-adic Eichler-Shimura cohomology groups}

We give here some preparatory results, in order to complete the proof of (1.5.5) in the next subsection and in the appendix. In accordance with the terminology of [16], we set

$$
\left\{\begin{array}{l}
\mathfrak{A}_{\infty}^{*}:=e^{*} E S_{p}(N)_{\mathbf{Z}_{p}}^{I_{p}}=e^{*} G E S_{p}(N)_{\mathbf{Z}_{p}}^{I_{p}} ; \mathfrak{A}_{\infty, \mathfrak{o}}^{*}:=\mathfrak{A}_{\infty}^{*} \widehat{\otimes} \mathbf{z}_{p} \mathfrak{o}=\mathfrak{A}_{\infty}^{*} \otimes_{\Lambda_{\mathbf{z}_{p}}} \Lambda_{\mathfrak{o}} \\
\mathfrak{B}_{\infty}^{*}:=e^{*} E S_{p}(N)_{\mathbf{Z}_{p}} / \mathfrak{A}_{\infty}^{*} ; \mathfrak{B}_{\infty, \mathfrak{o}}^{*}:=\mathfrak{B}_{\infty}^{*} \widehat{\otimes}_{\mathbf{z}_{p}} \mathfrak{o}=\mathfrak{B}_{\infty}^{*} \otimes_{\Lambda_{\mathbf{z}_{p}}} \Lambda_{\mathfrak{o}} \\
\widetilde{\mathfrak{B}}_{\infty}^{*}:=e^{*} G E S_{p}(N)_{\mathbf{z}_{p}} / \mathfrak{A}_{\infty}^{*} ; \widetilde{\mathfrak{B}}_{\infty, \mathfrak{o}}^{*}:=\widetilde{\mathfrak{B}}_{\infty}^{*} \widehat{\otimes}_{\mathbf{z}_{p}} \mathfrak{o}=\widetilde{\mathfrak{B}}_{\infty}^{*} \otimes_{\Lambda_{\mathbf{z}_{p}}} \Lambda_{\mathfrak{o}}
\end{array}\right.
$$

where $I_{p}$ is the inertia group of $\operatorname{Gal}\left(\overline{\mathbf{Q}}_{p} / \mathbf{Q}_{p}\right) \subset \operatorname{Gal}(\overline{\mathbf{Q}} / \mathbf{Q})$. In what follows, we indicate by the superscript " $(i)$ " the $\omega^{i}$-eigenspace with respect to the action of $(\mathbf{Z} / p \mathbf{Z})^{\times}$. Then, in general (i.e. without assuming that $p \nmid \varphi(N)$ ), we have the following:

THEOREM (3.4.2).-Assume that $i \not \equiv 0$ modulo $p-1$. Then a geometric Frobenius $\Phi_{p} \in \operatorname{Gal}\left(\overline{\mathbf{Q}}_{p} / \mathbf{Q}_{p}\right)$ acts as $T^{*}(p)$ on $\mathfrak{A}_{\infty}^{*(i)}$.

Proof. - We first note that when $N=1$, this is due to Mazur and Wiles [12], p. 250 (under a different choice of the models of modular curves).

If $J_{r}$ denotes the Jacobian variety of $X_{1}\left(N p^{r}\right)$ defined over $\mathbf{Q}$, there is a well-known isomorphism of Galois modules: $H^{1}\left(X_{1}\left(N p^{r}\right) \otimes_{\mathbf{Q}} \overline{\mathbf{Q}}, \mathbf{Z}_{p}\right) \cong T_{p}\left(J_{r}\right)(-1)$ through which $T^{*}(-)$ on the left-hand side commutes with $T(-)$ (the covariant action of the Hecke correspondence) on the right-hand side. Thus, what we need to show is that $\Phi_{p}$ acts as $T(p)$ on $e T_{p}\left(J_{r}\right)^{(i)}(-1)^{I_{p}}$ for each $r \geqslant 1$ when $i \not \equiv 0 \bmod p-1, e$ being Hida's idempotent associated with $T(p)$. For this, we may take $\Phi_{p}$ from $\operatorname{Gal}\left(\overline{\mathbf{Q}}_{p} / \mathbf{Q}_{p}\left(\zeta_{p^{r}}\right)\right)$.

Let $B_{r}$ be the "good quotient" of $J_{r}$ studied by Mazur and Wiles [11,12], Tilouine [20] and others (cf. [14], 3.2). It has good reduction over $\mathbf{Q}_{p}\left(\zeta_{p^{r}}\right)$, and we denote by $B_{r / \mathbf{Z}_{p}\left[\zeta_{p^{r}}\right]}$ (resp. $B_{r / \mathbf{F}_{p}}$ ) the Néron model of $B_{r}$ over $\mathbf{Z}_{p}\left[\zeta_{p^{r}}\right]$ (resp. its closed fibre). We can consider the $p$-divisible group over $\mathbf{Z}_{p}\left[\zeta_{p^{r}}\right]$

$$
G_{r}:=\left(e \cdot B_{r / \mathbf{z}_{p}\left[\zeta_{p^{r}}\right]}(p)\right)^{(i)}
$$

where " $(p)$ " means the associated $p$-divisible group, and we know that $e T_{p}\left(J_{r}\right)^{(i)} \cong T_{p}\left(G_{r}\right)$ canonically. On the other hand, if $q_{p}$ is a positive integer congruent to 1 (resp. $p$ ) mod $p^{r}$ (resp. $N$ ), we also know that $T(p)$ acts as $T\left(q_{p}, q_{p}\right) \Phi_{p}^{-1}$ on $e T_{p}\left(B_{r / \mathbf{F}_{p}}\right)$ (cf. Saby [17], Théorèmes 2.2.3 and 2.3.1).

Let $G_{r}^{0}$ (resp. $G_{r}^{\text {ét }}$ ) be the connected part (resp. the maximal étale quotient) of $G_{r}$. The results above imply that $T(p)=T\left(q_{p}, q_{p}\right) \Phi_{p}^{-1}$ on $T_{p}\left(G_{r}^{\text {ét }}\right)$. Let us denote by $[,]_{r}$ the twisted Weil pairing on $T_{p}\left(J_{r}\right)$, which induces a perfect duality between $T_{p}\left(G_{r}^{0}\right)$ and $T_{p}\left(G_{r}^{\text {ét }}\right)$ (cf. [14], (3.2.5) where (the "*-version" of) the results of Mazur, Wiles and Tilouine are reviewed, or [16], (1.2.8)). Now, with the choice of $\Phi_{p}$ as above, we have

$$
\left[x^{\Phi_{p}}, y^{\Phi_{p}}\right]_{r}=\left[T\left(q_{p}, q_{p}\right) T(p)^{-1} x^{\Phi_{p}}, y\right]_{r}
$$

for all $x \in e T_{p}\left(G_{r}^{0}\right)$ and $y \in T_{p}\left(G_{r}^{\text {et }}\right)$. On the other hand, the well-known property of the twisted Weil pairing implies that

$$
\left[x^{\Phi_{p}}, y^{\Phi_{p}}\right]_{r}=\kappa\left(\Phi_{p}\right)\left[T\left(q_{p}, q_{p}\right) x, y\right]_{r} .
$$

We thus conclude that $\Phi_{p}=\kappa\left(\Phi_{p}\right) T(p)$ on $T_{p}\left(G_{r}^{0}\right)$. Our result follows from this, since $e T_{p}\left(J_{r}\right)^{(i)}(-1)^{I_{p}}=T_{p}\left(G_{r}^{0}\right)(-1)$ (loc. cit.). 
In the rest of this section, we fix $(\theta, \psi)$ and $\mathfrak{r}$, and assume (1.5.2). For notational simplicity, we put:

$$
\left\{\begin{array}{l}
\mathfrak{h}^{*}:=e^{*} h^{*}(N ; \mathfrak{r})_{\mathfrak{M}^{*}}, \\
\mathfrak{H}^{*}:=e^{*} \mathcal{H}^{*}(N ; \mathfrak{r})_{\mathfrak{M}^{*}}, \\
X:=e^{*} E S_{p}(N)_{\mathfrak{r}, \mathfrak{M}^{*}}, \\
Y:=e^{*} G E S_{p}(N)_{\mathfrak{r}, \mathfrak{M}^{*}},
\end{array}\right.
$$

with $\mathfrak{M}^{*}=\mathfrak{M}^{*}(\theta, \psi)$. We then know that $X_{+}:=X^{I_{p}}=\mathfrak{A}_{\infty, \mathfrak{r}, \mathfrak{M}^{*}}^{*}$ is a free $\mathfrak{h}^{*}$-module of rank one, and $X / X_{+} \otimes_{\Lambda_{\mathfrak{r}}} \mathcal{L}_{k}$ is a free $\mathfrak{h}^{*} \otimes_{\Lambda_{\mathfrak{r}}} \mathcal{L}_{k}$-module of rank one ([16], (2.3.6), [15], (5.1.2)). We therefore obtain a two-dimensional Galois representation:

$$
\rho: \operatorname{Gal}(\overline{\mathbf{Q}} / \mathbf{Q}) \rightarrow G L_{\mathfrak{h}^{*}}(X) \hookrightarrow G L_{2}\left(\mathfrak{h}^{*} \otimes_{\Lambda_{\mathfrak{r}}} \mathcal{L}_{k}\right) .
$$

This is unramified outside $N p$, and its determinant is given by

$$
\operatorname{det} \rho(\sigma)=(\theta \psi \omega)^{-1}(\sigma)\langle\kappa(\sigma)\rangle^{-1} \iota(\langle\kappa(\sigma)\rangle)^{-1}
$$

for all $\sigma \in \operatorname{Gal}(\overline{\mathbf{Q}} / \mathbf{Q})$. In fact, for any prime number $l$ not dividing $N p$, we have $\operatorname{det} \rho\left(\Phi_{l}\right)=l T^{*}(l, l)$ for the geometric Frobenius at $l([15],(5.1 .5))$; and hence (3.1.1) and the Cebotarev density theorem gives the formula above.

Now we want to split the exact sequences:

$$
\left\{\begin{array}{l}
0 \rightarrow X_{+} \rightarrow X \rightarrow X / X_{+} \rightarrow 0, \\
0 \rightarrow X_{+} \rightarrow Y \rightarrow Y / X_{+} \rightarrow 0
\end{array}\right.
$$

of $\mathfrak{H}^{*}$-modules. For this, we define an integer $i \bmod (p-1)$ by

$$
\left.\theta\right|_{(\mathbf{Z} / p \mathbf{Z}) \times}=\omega^{i}
$$

Then, $\sigma \in I_{p}$ acts on $X / X_{+}$and $Y / X_{+}$as $\omega^{-i-1}(\sigma)\langle\kappa(\sigma)\rangle^{-1} \iota(\langle\kappa(\sigma)\rangle)^{-1}$, by [16], (1.3.8). When $i \not \equiv-1 \bmod (p-1)$, it is easy to split (3.4.6), and this was in fact done in [15], 5.3 and [16], 3.2: We take and fix a $\sigma_{0} \in I_{p}$ such that $\omega^{-i-1}\left(\sigma_{0}\right) \neq 1$ and $\left\langle\kappa\left(\sigma_{0}\right)\right\rangle=1$. Then we define $X_{-}$(resp. $Y_{-}$) as the subspace of $X$ (resp. $Y$ ) consisting of elements on which $\sigma_{0}$ acts as multiplication by $\omega^{-i-1}\left(\sigma_{0}\right)$. It is clear that this is mapped isomorphically onto $X / X_{+}$(resp. $\left.Y / X_{+}\right)$.

Next assume that $i \equiv-1 \bmod (p-1)$. In the following, we take $\Phi_{p}$ in such a way that $\kappa\left(\Phi_{p}\right)=1$, which is of course possible since $\mathbf{Q}_{p}\left(\zeta_{p^{\infty}}\right) / \mathbf{Q}_{p}$ is totally ramified at $p$. For this $\Phi_{p}$, we have $\operatorname{det} \rho\left(\Phi_{p}\right)=(\theta \psi \omega)(p)$.

LEMMA (3.4.8). - Assume that $i \equiv-1 \bmod (p-1)$. Then $T^{*}(p)-(\theta \psi \omega)(p) T^{*}(p)^{-1} \in \mathfrak{H}^{*}$ is a unit.

Proof. - Since $\mathfrak{H}^{*}$ is local, it is enough to show that the element above reduced modulo the maximal ideal $\mathfrak{M}^{*}$ is a unit. But since $T^{*}(p) \equiv \psi(p) \bmod \mathfrak{M}^{*}$, we see that it is congruent to $\psi(p)-(\theta \omega)(p) \bmod \mathfrak{M}^{*}$. Our assumption (1.5.2) then implies that this is a unit.

By (3.4.2), $\Phi_{p}$ acts as $T^{*}(p)$ on $X_{+}$, and by the remark above, it acts as $(\theta \psi \omega)(p) T^{*}(p)^{-1}$ on $X / X_{+}$. Thus if we set $X_{-}:=\left(\right.$the elements of $X$ on which $\Phi_{p}$ acts as $\left.(\theta \psi \omega)(p) T^{*}(p)^{-1}\right)$, then this is a direct summand of $X$ which gives the splitting of the first sequence in (3.4.6), by the lemma above. 
To treat the second sequence in (3.4.6), we recall that there are exact sequences of $\operatorname{Gal}(\overline{\mathbf{Q}} / \mathbf{Q})$ and $\operatorname{Gal}\left(\overline{\mathbf{Q}}_{p} / \mathbf{Q}_{p}\right)$-modules, respectively:

$$
\left\{\begin{array}{l}
0 \rightarrow X \rightarrow Y \rightarrow \mathcal{C}_{p}(N)_{\mathfrak{r}, \mathfrak{M}^{*}}(-1) \rightarrow 0, \\
0 \rightarrow X / X_{+} \rightarrow Y / X_{+} \rightarrow \mathcal{C}_{p}(N)_{\mathfrak{r}, \mathfrak{M}^{*}}(-1) \rightarrow 0
\end{array}\right.
$$

([15], 4.3). Here, we have $\mathcal{C}_{p}(N)_{\mathfrak{r}, \mathfrak{M}^{*}}=\Lambda_{\mathfrak{r}}\left(e^{*} \mathbf{e}_{\infty}\right)$, by (2.3.6) and (3.1.2). The main result of [16] implies that the second sequence splits uniquely as $\mathfrak{H}^{*}$ - and $\operatorname{Gal}\left(\overline{\mathbf{Q}}_{p} / \mathbf{Q}_{p}\right)$-modules when tensored with $\mathcal{L}_{k}$ over $\Lambda_{\mathfrak{r}}$. Thus we can define $Y_{-} \subseteq Y$ in exactly the same manner as $X_{-}$to get the desired splitting of $\mathfrak{H}^{*}$-modules, for the same reason as above, with the following:

LEMMA (3.4.10). - When $i \equiv-1 \bmod (p-1), \Phi_{p}\left(\right.$ such that $\left.\kappa\left(\Phi_{p}\right)=1\right)$ acts on $\mathcal{C}_{p}(N)_{\mathfrak{r}, \mathfrak{M}^{*}}$ as $(\theta \psi \omega)(p) T^{*}(p)^{-1}$, and hence its action on $Y / X_{+}$is also given by $(\theta \psi \omega)(p) T^{*}(p)^{-1}$.

Proof. - Since $T^{*}(p)=\psi(p)$ on $\Lambda_{\mathfrak{r}}\left(e^{*} \mathbf{e}_{\infty}\right)$, it is enough to show that

$$
\mathbf{e}_{r}^{\Phi_{p}}=(\theta \omega)(p) \mathbf{e}_{r}
$$

for each $r \geqslant 1$.

In general, if $\sigma \in \operatorname{Gal}(\overline{\mathbf{Q}} / \mathbf{Q})$ satisfies $\zeta_{N_{r}}^{\sigma}=\zeta_{N_{r}}^{\alpha}$ with an integer $\alpha$, and if $\alpha^{\prime} \in \mathbf{Z}$ is taken so that $\alpha \alpha^{\prime} \equiv 1 \bmod N_{r}$, then $\sigma$ sends the cusp $\left[\begin{array}{c}a \\ c\end{array}\right]_{N_{r}} \bmod \{ \pm 1\}$ to $\left[\begin{array}{c}a \\ \alpha^{\prime} c\end{array}\right]_{N_{r}} \bmod \{ \pm 1\}$ (Stevens [19], Theorem 1.3.1). For $\sigma=\Phi_{p}$, we clearly have $\alpha^{\prime} \equiv p \bmod N^{r}$ and $\alpha^{\prime} \equiv 1$ $\bmod p^{r}$, and the conclusion follows from the definition of $\mathbf{e}_{r}$ (cf. (2.2.2) and (2.2.6)).

Summing up, we have obtained the direct sum decomposition of $\mathfrak{H}^{*}$-modules:

$$
\left\{\begin{array}{l}
X=X_{+} \oplus X_{-} \\
Y=X_{+} \oplus Y_{-}
\end{array}\right.
$$

\subsection{Congruence modules attached to $p$-adic Eichler-Shimura cohomology groups}

In this subsection, we treat the cases (III) and (IV), and complete the proof of (1.5.5). We keep the notation and the assumption of Section 3.4.

We first consider the exact sequence already mentioned above:

$$
0 \rightarrow X \rightarrow Y \rightarrow \Lambda_{\mathfrak{r}}\left(e^{*} \mathbf{e}_{\infty}\right)(-1) \rightarrow 0
$$

This is the sequence (III) in (1.5.5). From the existence of $Y_{-}$above, we see that this exact sequence splits uniquely as modules over $\mathfrak{H}^{*}$ when tensored with $\mathcal{L}_{k}$ over $\Lambda_{\mathfrak{r}}$, and we want to determine the structure of the congruence module attached to this situation. 
For this, we consider the following commutative diagram in which all the sequences are exact:

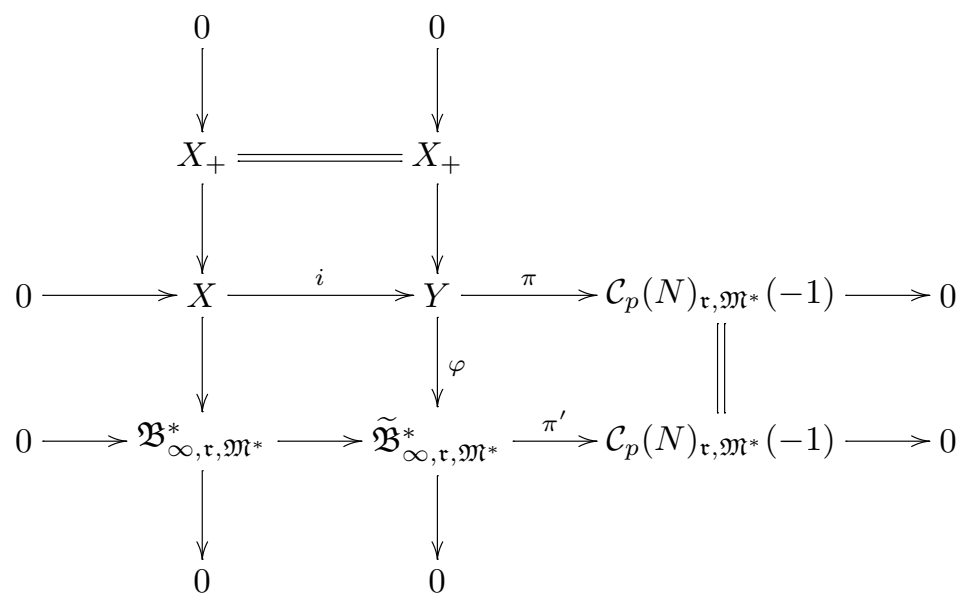

Let

$$
s: \mathcal{C}_{p}(N)_{\mathfrak{r}, \mathfrak{M}^{*}}(-1) \otimes_{\Lambda_{\mathfrak{r}}} \mathcal{L}_{k} \rightarrow Y \otimes_{\Lambda_{\mathfrak{r}}} \mathcal{L}_{k}
$$

and

$$
s^{\prime}: \mathcal{C}_{p}(N)_{\mathfrak{r}, \mathfrak{M}^{*}}(-1) \otimes_{\Lambda_{\mathfrak{r}}} \mathcal{L}_{k} \rightarrow \widetilde{\mathfrak{B}}_{\infty, \mathfrak{r}, \mathfrak{M}^{*}} \otimes_{\Lambda_{\mathfrak{r}}} \mathcal{L}_{k}
$$

be the homomorphisms giving the (unique) splittings of the two horizontal lines above, as $\mathfrak{H}^{*}$ modules.

The uniqueness implies that we have: $\varphi \circ s=s^{\prime}$. We also have that $s$ and $s^{\prime}$ commute with the action of $\operatorname{Gal}\left(\overline{\mathbf{Q}}_{p} / \mathbf{Q}_{p}\right)$. Thus from the construction in the previous subsection, we see that $s\left(\mathcal{C}_{p}(N)_{\mathfrak{r}, \mathfrak{M}^{*}}(-1)\right) \subseteq Y_{-} \otimes_{\Lambda_{\mathfrak{r}}} \mathcal{L}_{k}$. Consequently, the congruence modules attached to two horizontal lines above are isomorphic.

As before, we take $K(\supseteq k)$ so large that it contains all the roots of unity. By the main result of [16], we then have the following commutative diagram:

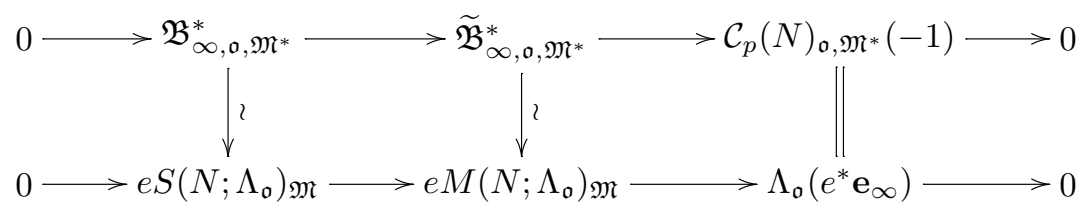

where we are fixing an isomorphism: $\mathbf{Z}_{p}(-1) \cong \mathbf{Z}_{p}$. Here, the Hecke operator $T^{*}(-)$ on the upper left two modules corresponds to $T(-)$ on the lower left two modules. The case (I) of (1.5.5), which was established in Section 3.1, together with (1.1.7) implies that the congruence module attached to the lower sequence is isomorphic to $\Lambda_{\mathfrak{o}} /(A(T ; \theta, \psi))$ if $(\theta, \psi) \neq\left(\omega^{-2}, \mathbf{1}\right)$ and $\{0\}$ otherwise. (Remember that $\Lambda_{\mathfrak{o}}$ is faithfully flat over $\Lambda_{\mathfrak{r}}$.) The case (III) of (1.5.5) therefore follows from this combined with (1.1.7) and (1.1.9).

Let us finally treat the case (IV) of (1.5.5). In Section 1.3, we have established the duality (1.3.3) between $e^{*} G E S_{p}(N)_{\mathfrak{r}}$ and $e^{*} M S_{p}(N)_{\mathfrak{r}}$. By (1.3.5), this induces the self-duality of 
$e^{*} E S_{p}(N)_{\mathfrak{r}}$. Thus taking the $\Lambda_{\mathfrak{r}}$-dual of the exact sequence:

$$
0 \rightarrow e^{*} E S_{p}(N)_{\mathfrak{r}} \stackrel{i}{\rightarrow} e^{*} G E S_{p}(N)_{\mathfrak{r}} \rightarrow \mathcal{C}_{p}(N)_{\mathfrak{r}}(-1) \rightarrow 0
$$

we obtain an exact sequence:

$$
0 \rightarrow \mathcal{C}_{p}(N)_{\mathfrak{r}}(-1)^{\vee} \rightarrow e^{*} M S_{p}(N)_{\mathfrak{r}} \stackrel{\pi}{\rightarrow} e^{*} E S_{p}(N)_{\mathfrak{r}} \rightarrow 0
$$

where $\pi$ is the natural surjection. We have especially shown that $B:=\operatorname{Ker}(\pi)$ is a free $\Lambda_{\mathfrak{r}}$-module, in general. From (1.3.3), (ii), we have a natural action of $e^{*} \mathcal{H}^{*}(N ; \mathfrak{r})$ on $e^{*} M S_{p}(N)_{\mathfrak{r}}$, which induces the original action of $e^{*} h^{*}(N ; \mathfrak{r})$ on the quotient $e^{*} E S_{p}(N)_{\mathfrak{r}}$. Now taking the $\Lambda_{\mathfrak{r}}$-dual of the diagram (3.5.2), we obtain:

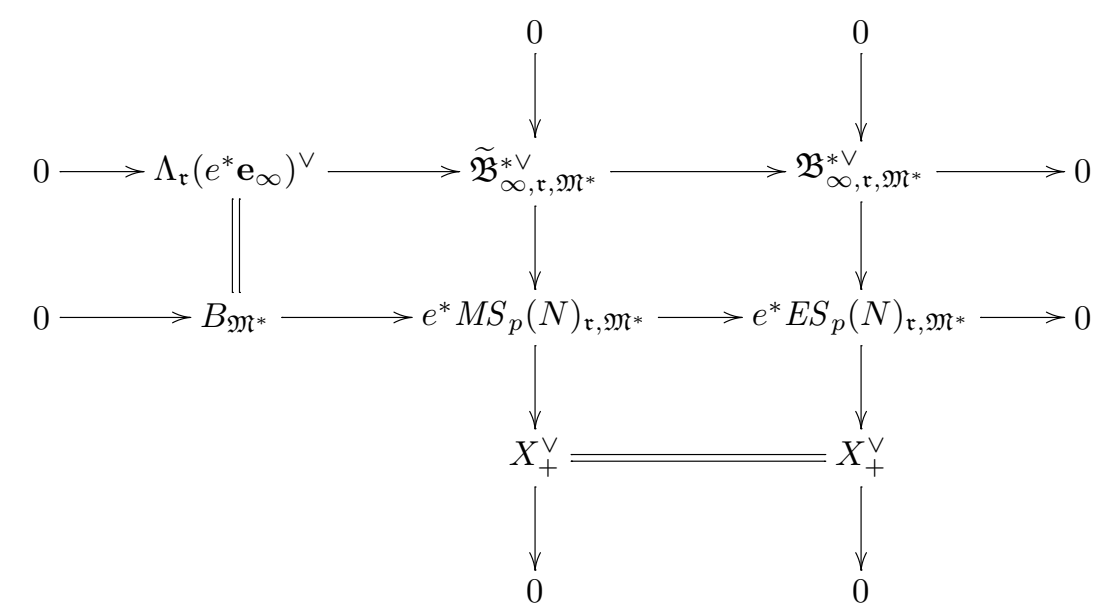

The middle horizontal sequence is the one labelled as (IV) in (1.5.5). Here, we already know that the two horizontal sequences split uniquely as modules over $\mathfrak{H}^{*}$ when tensored with $\mathcal{L}_{k}$ over $\Lambda_{\mathfrak{r}}$.

When $(\theta, \psi)=\left(\omega^{-2}, \mathbf{1}\right)$, we already know that the upper horizontal exact sequence in (3.5.2) splits as modules over $\mathfrak{H}^{*}$, and hence the same holds for the lower horizontal sequence in (3.5.6). This shows the vanishing of the congruence module in question in this case. We may thus assume that $(\theta, \psi) \neq\left(\omega^{-2}, \mathbf{1}\right)$.

As in Section 3.4, we choose a geometric Frobenius $\Phi_{p} \in \operatorname{Gal}\left(\overline{\mathbf{Q}}_{p} / \mathbf{Q}_{p}\right)$ such that $\kappa\left(\Phi_{p}\right)=1$ in the following:

LEMMA (3.5.7). - Let the integer $i \bmod (p-1)$ be defined by (3.4.7). Through the natural action of $\operatorname{Gal}(\overline{\mathbf{Q}} / \mathbf{Q})$ on $e^{*} M S_{p}(N)_{\mathfrak{r}}, \sigma \in I_{p}$ acts trivially on $\widetilde{\mathfrak{B}}_{\infty, \mathfrak{r}, \mathfrak{M}^{*}}^{* \vee}$ and as $\omega^{-i-1}(\sigma)\langle\kappa(\sigma)\rangle^{-1} \iota\left(\langle\kappa(\sigma)\rangle^{-1}\right)$ on $X_{+}^{\vee}$. When $i \equiv-1 \bmod (p-1), \Phi_{p}$ acts as $T^{*}(p)$ on $\widetilde{\mathfrak{B}}_{\infty, \mathfrak{r}, \mathfrak{M}^{*}}^{*}$, and as $(\theta \psi \omega)(p) T^{*}(p)^{-1}$ on $X_{+}^{\vee}$, respectively.

Proof. - This directly follows from the known facts for $e^{*} G E S_{p}(N)_{\mathfrak{r}, \mathfrak{M}^{*}}((3.4 .2)$, (3.4.10) and [16], (1.3.8)) and (1.3.3).

It easily follows from this that the congruence modules (of the form " $t(B) / A$ ") attached to two horizontal sequences in (3.5.6) are isomorphic. On the other hand, by (3.5.3) and (3.3.1), the upper horizontal sequence in (3.5.6) tensored with $\Lambda_{\mathfrak{o}}$ over $\Lambda_{\mathfrak{r}}$ can be described in terms of 
Hecke algebras:

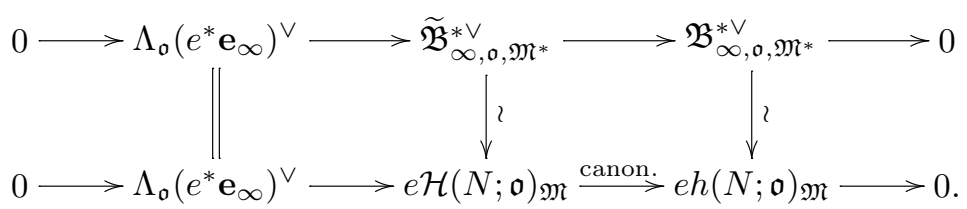

Now fix an isomorphism $\Lambda_{\mathfrak{r}}\left(e^{*} \mathbf{e}_{\infty}\right)^{\vee} \cong \Lambda_{\mathfrak{r}}$. Let $t: \widetilde{\mathfrak{B}}_{\infty, \mathfrak{r}, \mathfrak{M}^{*}}^{* \vee} \otimes_{\Lambda_{\mathfrak{r}}} \mathcal{L}_{k} \rightarrow \mathcal{L}_{k}$ be the homomorphism giving the splitting of the exact sequence in question, and $t^{\prime}: \widetilde{\mathfrak{B}}_{\infty, \mathfrak{o}, \mathfrak{M}^{*}}^{* \vee} \otimes_{\Lambda_{\mathfrak{o}}} \mathcal{L}_{K} \rightarrow \mathcal{L}_{K}$ its $\mathcal{L}_{K}$-linear extension. Then, from the diagram above and the argument in Section 3.2, we see that $t^{\prime}\left(\widetilde{\mathfrak{B}}_{\infty, \mathfrak{o}, \mathfrak{M}^{*}}^{* V}\right)$ is a free $\Lambda_{\mathfrak{o}}$-module of rank one. In addition, we see from the case (II) of (1.5.5) and (1.1.7) that the congruence module attached to (3.5.8) is isomorphic to $\Lambda_{\mathfrak{o}} /(A(T ; \theta, \psi))$. These imply that

$$
t^{\prime}\left(\widetilde{\mathfrak{B}}_{\infty, \mathfrak{o}, \mathfrak{M}^{*}}^{* \vee}\right)=\frac{1}{A(T ; \theta, \psi)} \Lambda_{\mathfrak{o}}
$$

which in turn implies that

$$
A(T ; \theta, \psi) t\left(\widetilde{\mathfrak{B}}_{\infty, \mathfrak{r}, \mathfrak{M}^{*}}^{* \vee} \subseteq \Lambda_{\mathfrak{r}} .\right.
$$

But if the equality does not hold here, the left-hand side must be contained in the maximal ideal $(T, \varpi)$ of $\Lambda_{\mathfrak{r}}$, which evidently contradicts (3.5.9).

This settles the case (IV), and we have completed the proof of our main result (1.5.5).

Remark (3.5.10). - In [1], Greenberg and Stevens considered the congruence module attached to (3.5.5) (with its canonical splitting), and raised the problem of analyzing its structure.

More precisely, the relation between the terminology of [1] and ours is as follows: The module $\mathbf{D}$ in [1] is isomorphic to $\mathbf{Z}_{p} \llbracket\left(\mathbf{Z}_{p}^{2}\right)^{\prime} \rrbracket$ with

$$
\left(\mathbf{Z}_{p}^{2}\right)^{\prime}:=\left\{[a, c] \mid a, c \in \mathbf{Z}_{p}, a \mathbf{Z}_{p}+c \mathbf{Z}_{p}=\mathbf{Z}_{p}\right\},
$$

and these are acted on by $M_{2}\left(\mathbf{Z}_{p}\right)$ from the right ([1], p. 431). Let

$$
Z_{0}:=\left\{\left[\begin{array}{l}
a \\
c
\end{array}\right] \mid a, c \in \mathbf{Z}_{p}, a \mathbf{Z}_{p}+c \mathbf{Z}_{p}=\mathbf{Z}_{p}\right\} .
$$

Then the obvious bijection $\left(\mathbf{Z}_{p}^{2}\right)^{\prime} \leftrightarrow Z_{0}$ given by $[a, c] \leftrightarrow\left[\begin{array}{c}-c \\ a\end{array}\right]$ transforms the above right action of $g \in M_{2}\left(\mathbf{Z}_{p}\right)$ to the left action of $g^{*}$ on $Z_{0}$ defined in a similar manner as in [1], where $*$ denotes the canonical involution. Via this isomorphism, we may identify $\operatorname{Symb}_{\Gamma_{1}(N)}(\mathbf{D})$ with $\operatorname{Hom}_{\Gamma_{1}(N)}\left(D_{0}, \mathbf{Z}_{p} \llbracket Z_{0} \rrbracket\right)=M S_{p}(N) \mathbf{z}_{p}$ (cf. [14], (1.2.12)), and hence the exact sequence (6.7) in [1] with

$$
0 \rightarrow \operatorname{Ker}(\text { canon. }) \rightarrow M S_{p}(N) \mathbf{z}_{p} \stackrel{\text { canon. }}{\longrightarrow} E S_{p}(N) \mathbf{z}_{p} \rightarrow 0 \text {. }
$$

On the other hand, in [1], the Hecke operators $T_{n}$ and $[m]$ are considered. Here, $n$ and $m$ are positive integers and $m$ is prime to $N p$. Let $W_{N}:=\left[\begin{array}{cc}0 & -1 \\ N & 0\end{array}\right]$. For $f \in \operatorname{Hom}_{\Gamma_{1}(N)}\left(D_{0}, \mathbf{Z}_{p} \llbracket Z_{0} \rrbracket\right)$, we define $f \mid W_{N}$ by $f \mid W_{N}(d):=W_{N}^{*} f\left(W_{N} d\right)$. It is then clear that the correspondence: $f \mapsto f \mid W_{N}$ gives an automorphism of $\operatorname{Hom}_{\Gamma_{1}(N)}\left(D_{0}, \mathbf{Z}_{p} \llbracket Z_{0} \rrbracket\right)$. A direct calculation, using results of [13], 7.3 and 7.4, shows that the conjugation by this automorphism transforms $T_{n}$ 
(resp. $[m]$ ) to our $T^{*}(n)$ (resp. $T^{*}(m, m)$ ). It follows that the module $C$ defined on [1], p. 444 is isomorphic to the congruence module attached to (3.5.5) with $\mathfrak{r}=\mathbf{Z}_{p}$ as a $\Lambda_{\mathbf{z}_{p}}$-module.

We have determined the structure of the localizations of the congruence module attached to (3.5.5) at "primitive" (in the sense that $u v=N$ or $N p$ ) and non-exceptional $\mathfrak{M}^{*}(\theta, \psi)$ when $p \nmid \varphi(N)$, which gives a partial solution to the problem of Greenberg and Stevens.

\section{Appendix A. Construction of abelian $p$-extensions over $\mathbf{Z}_{p}$-extensions}

\section{A.1. The extension $L / F_{\infty}$}

We keep the notation of the text. Especially, we fix $p, N$ and $(\theta, \psi)$, and assume (1.5.2) for them. $\mathfrak{r}$ is the ring of integers of a finite extension $k$ of $\mathbf{Q}_{p}$ containing the values of $\theta$ and $\psi$.

In [15], Section 5 and [16], Section 3, we applied the method of Harder and Pink [2] and Kurihara [9] to $e^{*} E S_{p}(N)_{\mathfrak{r}}$ to construct large unramified abelian pro- $p$ extensions over cyclotomic $\mathbf{Z}_{p}$-extensions of abelian number fields, when $\psi=\mathbf{1}$. The purpose of this appendix is to show that one can apply the same technique for general $(\theta, \psi)$ (such that $\theta \psi^{-1} \neq \omega^{-2}$ ) as above, which will also settle the case (II) of (1.5.5). When the argument is parallel to the above mentioned works, we will be brief, referring the details to them.

In the following, we argue under the assumption that the ideal $\mathfrak{m}^{*}:=\mathfrak{m}^{*}(\theta, \psi)$ defined in (1.5.1) is a proper ideal of the Hecke algebra $e^{*} h^{*}(N ; \mathfrak{r})$, which assures us that

$$
\mathfrak{h}^{*}:=e^{*} h^{*}(N ; \mathfrak{r})_{\mathfrak{M}^{*}}=e^{*} h^{*}(N ; \mathfrak{r})_{\mathfrak{m}}
$$

is not a zero ring. As for (1.5.5), (II), when $(\theta, \psi)=\left(\omega^{-2}, \mathbf{1}\right)$, we will soon arrive at a contradiction to this assumption, while otherwise, we have reduced the problem to this case in Section 3.2.

Now recall that we have set $X:=e^{*} E S_{p}(N)_{\mathfrak{r}, \mathfrak{M}^{*}}=e^{*} E S_{p}(N)_{\mathfrak{r}, \mathfrak{m}^{*}}$, and that $X$ is a direct sum of $X_{+}:=X^{I_{p}}$ and $X_{-}$defined in Section 3.4. We know that $X_{-} \otimes_{\Lambda_{\mathfrak{r}}} \mathcal{L}_{k}$ (resp. $X_{+}$) is a free $\mathfrak{h}^{*} \otimes_{\Lambda_{\mathfrak{r}}} \mathcal{L}_{k}$-module (resp. free $\mathfrak{h}^{*}$-module) of rank one. We fix bases of these modules in this order, and realize the Galois representation $\rho$ (3.4.4) in the form:

$$
\rho(\sigma)=\left[\begin{array}{ll}
a(\sigma) & b(\sigma) \\
c(\sigma) & d(\sigma)
\end{array}\right] \in G L_{2}\left(\mathfrak{h}^{*} \otimes_{\Lambda_{\mathfrak{r}}} \mathcal{L}_{k}\right) .
$$

Thus by construction,

$$
\rho(\sigma)=\left[\begin{array}{cc}
\operatorname{det} \rho(\sigma) & 0 \\
* & 1
\end{array}\right] \quad \text { if } \sigma \in I_{p}
$$

and recall that $\operatorname{det} \rho$ is in general given by (3.4.5).

Let $J^{*}$ be the ideal of $\mathfrak{h}^{*}$ generated by $T^{*}(p)-\psi(p)$ and all $T^{*}(l)-(\theta(l) l \iota(\langle l\rangle)+\psi(l))$ for prime numbers $l$ not dividing $N p$. This is clearly contained in $I^{*}:=I^{*}(\theta, \psi) \mathfrak{h}^{*}$. Noting that, when $i \equiv-1 \bmod (p-1)$,

$$
\rho\left(\Phi_{p}\right)=\left[\begin{array}{cc}
(\theta \psi \omega)(p) T^{*}(p)^{-1} & 0 \\
0 & T^{*}(p)
\end{array}\right] \equiv\left[\begin{array}{cc}
(\theta \omega)(p) & 0 \\
0 & \psi(p)
\end{array}\right] \quad \bmod J^{*}
$$

for a geometric Frobenius at $p$ such that $\kappa\left(\Phi_{p}\right)=1$, we have the following: 
Lemma (A.1.3). - For any $\sigma, \tau \in \operatorname{Gal}(\overline{\mathbf{Q}} / \mathbf{Q})$, the elements

$$
a(\sigma)-\operatorname{det} \rho(\sigma) \psi(\sigma), \quad d(\sigma)-\psi(\sigma)^{-1} \quad \text { and } \quad b(\sigma) c(\tau)
$$

are contained in $J^{*}$.

The sets

$$
\{a(\sigma)-\operatorname{det} \rho(\sigma) \psi(\sigma) \mid \sigma \in \operatorname{Gal}(\overline{\mathbf{Q}} / \mathbf{Q})\}
$$

and

$$
\left\{d(\sigma)-\psi(\sigma)^{-1} \mid \sigma \in \operatorname{Gal}(\overline{\mathbf{Q}} / \mathbf{Q})\right\}
$$

generate the same ideal $J_{0}^{*}$ of $\mathfrak{h}^{*}$. If $i \not \equiv 0 \bmod (p-1)$, we in fact have $J_{0}^{*}=J^{*}$.

Definition (A.1.4). - We denote by $B$ and $C$ the $\mathfrak{h}^{*}$-submodule of $\mathfrak{h}^{*} \otimes_{\Lambda_{\mathfrak{r}}} \mathcal{L}_{k}$ generated by all $b(\sigma)$ and $c(\sigma)$ for $\sigma \in \operatorname{Gal}(\overline{\mathbf{Q}} / \mathbf{Q})$, respectively.

From the lemma above, $B C$ is an ideal of $\mathfrak{h}^{*}$ contained in $J^{*}$.

LEMMA (A.1.5). $-B C$ contains $T^{*}(l)-(\theta(l) l \iota(\langle l\rangle)+\psi(l))$ for infinitely many primes $l$ not dividing $N p$. If $i \not \equiv 0,-1 \bmod (p-1)$, then $B C$ in fact coincides with $J^{*}$. As a consequence, $B$ and $C$ are faithful $\mathfrak{h}^{*}$-modules.

Now consider the mapping defined by

$$
\varphi(\sigma):=\left[\begin{array}{cc}
\overline{\operatorname{det} \rho}(\sigma) \bar{\psi}(\sigma) & \bar{b}(\sigma) \\
0 & \bar{\psi}(\sigma)^{-1}
\end{array}\right],
$$

where the bar indicates the reduction modulo $I^{*}: \overline{\operatorname{det} \rho}(\sigma), \bar{\psi}(\sigma) \in \mathfrak{h}^{*} / I^{*}$ and $\bar{b}(\sigma) \in B / I^{*} B$. Under the matrix product in the obvious sense, $\varphi$ gives a representation of $\operatorname{Gal}(\overline{\mathbf{Q}} / \mathbf{Q})$.

Let $F$ be the abelian extension of $\mathbf{Q}$ corresponding to the intersection of the kernels of $\theta \omega$ and $\psi$. From our assumption, the degree of $F$ over $\mathbf{Q}$ is prime to $p$. Let $F_{\infty}$ be the cyclotomic $\mathbf{Z}_{p}$-extension of $F$, and as usual, we set

$$
\left\{\begin{array}{l}
\Delta:=\operatorname{Gal}(F / \mathbf{Q}), \\
\Gamma:=\operatorname{Gal}\left(F_{\infty} / F\right),
\end{array}\right.
$$

so that we have an identification: $\operatorname{Gal}\left(F_{\infty} / \mathbf{Q}\right)=\Delta \times \Gamma$. On the other hand, let

$$
\left\{\begin{array}{l}
L_{0}:=(\text { the field corresponding to } \operatorname{Ker}(\varphi)) \\
L:=L_{0} \cdot F_{\infty} \\
K:=(\text { the field corresponding to }\{\sigma \in \operatorname{Gal}(\overline{\mathbf{Q}} / \mathbf{Q}) \mid \overline{\operatorname{det} \rho}(\sigma)=1, \bar{\psi}(\sigma)=1\}) .
\end{array}\right.
$$

Clearly, $L / F_{\infty}$ is a pro- $p$ abelian extension, and $K \subseteq F_{\infty}$. It is easy to see that $K \supseteq F$. From (A.1.2), we see that the primes above $p$ are unramified in $L_{0} / K$, while the primes above $p$ ramify totally in $F_{\infty} / F$. We therefore obtain

$$
\operatorname{Gal}\left(L_{0} / K\right) \cong \operatorname{Gal}\left(L / F_{\infty}\right) \hookrightarrow B / I^{*} B
$$

by $\sigma \mapsto \bar{b}(\sigma)$.

$\operatorname{Gal}\left(F_{\infty} / \mathbf{Q}\right)$ acts on $\operatorname{Gal}\left(L / F_{\infty}\right)$ via the conjugation. Recall that we have set $\xi:=\theta \psi^{-1}$ (1.5.3), in the following: 
Lemma (A.1.10). - Identify $\operatorname{Gal}\left(L / F_{\infty}\right)$ with its image in $B / I^{*} B$ via (A.1.9). Then on this module, the action of $\Delta$ is given by the character $(\xi \omega)^{-1}$, and the action of $\gamma \in \Gamma$ is given by $\langle\kappa(\gamma)\rangle^{-1} \iota\left(\langle\kappa(\gamma)\rangle^{-1}\right)$.

Let $\mathfrak{r}_{0}$ be the ring generated by the values of $\xi$ over $\mathbf{Z}_{p}$. The lemma above implies that the image of $\operatorname{Gal}\left(L / F_{\infty}\right)$ is a $\Lambda_{\mathfrak{r}_{0}}$-submodule of $B / I^{*} B$. A computation of some commutator shows that the image of $\operatorname{Gal}\left(L / F_{\infty}\right)$ in fact generates $B / I^{*} B$ as a $\Lambda_{\mathfrak{r}}$-module; i.e. we have a surjection of $\Lambda_{\mathfrak{r}}$-modules

$$
\operatorname{Gal}\left(L / F_{\infty}\right) \otimes_{\mathfrak{r}_{0}} \mathfrak{r} \rightarrow B / I^{*} B .
$$

Now consider the case where $(\theta, \psi)=\left(\omega^{-2}, \mathbf{1}\right)$. Then $L$ is an unramified abelian pro- $p$ extension of the cyclotomic $\mathbf{Z}_{p}$-extension of $\mathbf{Q}\left(\zeta_{p}\right)$, whose Galois group is acted on by $\operatorname{Gal}\left(\mathbf{Q}\left(\zeta_{p}\right) / \mathbf{Q}\right)$ via the character $\omega$. As is well-known, such an extension must be trivial, and hence (A.1.11) forces $B$ to be $\{0\}$. This contradiction implies $I^{*}(\theta, \psi)=e^{*} h^{*}(N ; \mathfrak{r})$, which completes the proof of (1.5.5), (II) in this case.

We thus henceforth assume that $(\theta, \psi) \neq\left(\omega^{-2}, \mathbf{1}\right)$, and note that the power series $A(T ; \theta, \psi)$ belongs to $\Lambda_{\mathfrak{r}_{0}}$. The lemma above states that the action of $\mathbf{Z}_{p} \llbracket \Delta \times \Gamma \rrbracket$ on $\operatorname{Gal}\left(L / F_{\infty}\right)$ factors through $\mathfrak{r}_{0} \llbracket \Gamma \rrbracket$ via $(\xi \omega)^{-1}$. We fix a topological generator $\gamma_{0}$ of $\Gamma$ and let it correspond to a topological generator $\kappa\left(\gamma_{0}\right)=: u_{0}$ of $U_{1}$, which gives $\mathfrak{r}_{0} \llbracket \Gamma \rrbracket \cong \Lambda_{\mathfrak{r}_{0}}$, the latter being identified with $\mathfrak{r}_{0} \llbracket T \rrbracket$ via $u_{0} \leftrightarrow 1+T$. The resulting (Iwasawa theoretic) $\Lambda_{\mathfrak{r}_{0}}$-module structure of $\operatorname{Gal}\left(L / F_{\infty}\right)$ and the older one are transformed to each other by the (involutive) $\mathfrak{r}_{0}$-automorphism

$$
1+T \leftrightarrow u_{0}^{-1}(1+T)^{-1}
$$

of $\Lambda_{\mathfrak{r}_{0}}=\mathfrak{r}_{0} \llbracket T \rrbracket$. In view of this, we set

$$
\left\{\begin{array}{l}
F\left(T, \xi \omega^{2}\right):=G\left(u_{0}^{-1}(1+T)^{-1}-1, \xi \omega^{2}\right), \\
B(T ; \theta, \psi):=\left(\prod_{\substack{l \mid N \\
l \nmid \operatorname{cond}(\xi)}}\left((1+T)^{s(l)}-(\xi \omega)(l) l\right)\right) F\left(T, \xi \omega^{2}\right)
\end{array}\right.
$$

so that $F\left(u_{0}^{s}-1, \xi \omega^{2}\right)=L_{p}\left(s, \xi \omega^{2}\right)$ (cf. (1.4.5)), and $B(T ; \theta, \psi)$ generates the same ideal of $\Lambda_{\mathfrak{r}_{0}}$ as $A\left(u_{0}^{-1}(1+T)^{-1}-1 ; \theta, \psi\right)$.

THEOREM (A.1.13). - If we consider $\operatorname{Gal}\left(L / F_{\infty}\right)$ as a $\Lambda_{\mathfrak{r}_{0}}$-module Iwasawa theoretically, then we have

$$
\left\{\begin{array}{l}
\operatorname{Fitt}_{\Lambda_{\mathfrak{r}_{0}}}\left(\operatorname{Gal}\left(L / F_{\infty}\right)\right) \subseteq B(T ; \theta, \psi) \Lambda_{\mathfrak{r}_{0}}, \\
\operatorname{char}_{\Lambda_{\mathfrak{r}_{0}}}\left(\operatorname{Gal}\left(L / F_{\infty}\right)\right) \subseteq B(T ; \theta, \psi) \Lambda_{\mathfrak{r}_{0}},
\end{array}\right.
$$

where $\operatorname{Fitt}_{\Lambda_{\mathrm{r}_{0}}}$ (resp. char $_{\Lambda_{\mathrm{r}_{0}}}$ ) means the Fitting ideal (resp. the characteristic ideal) of a $\Lambda_{\mathfrak{r}_{0}}$-module.

Proof. - The first assertion is equivalent to saying that

$$
\operatorname{Fitt}_{\Lambda_{\mathrm{r}_{0}}}\left(\operatorname{Gal}\left(L / F_{\infty}\right)\right) \subseteq A(T ; \theta, \psi) \Lambda_{\mathfrak{r}_{0}}
$$

with respect to the older description of the $\Lambda_{\mathfrak{r}_{0}}$-module structure above, which we now propose to prove.

First note that, from the definition of the Fitting ideal, we have

$$
\operatorname{Fitt}_{\Lambda_{\mathfrak{r}_{0}}}\left(\operatorname{Gal}\left(L / F_{\infty}\right)\right) \Lambda_{\mathfrak{r}}=\operatorname{Fitt}_{\Lambda_{\mathfrak{r}}}\left(\operatorname{Gal}\left(L / F_{\infty}\right) \otimes_{\mathfrak{r}_{0}} \mathfrak{r}\right)
$$


But it follows from (A.1.11) that

$$
\operatorname{Fitt}_{\Lambda_{\mathfrak{r}}}\left(\operatorname{Gal}\left(L / F_{\infty}\right) \otimes_{\mathfrak{r}_{0}} \mathfrak{r}\right) \subseteq \operatorname{Fitt}_{\Lambda_{\mathfrak{r}}}\left(B / I^{*} B\right)
$$

Let $\mathfrak{h}^{*} / I^{*}=\Lambda_{\mathfrak{r}} / \mathfrak{a}$ with an ideal $\mathfrak{a}$ of $\Lambda_{\mathfrak{r}}$. (A.1.5), (3.1.9) and a standard argument imply

$$
\operatorname{Fitt}_{\Lambda_{\mathfrak{r}}}\left(B / I^{*} B\right) \subseteq \mathfrak{a} \subseteq A(T ; \theta, \psi) \Lambda_{\mathfrak{r}}
$$

Combining these, we have

$$
\operatorname{Fitt}_{\Lambda_{\mathfrak{r}_{0}}}\left(\operatorname{Gal}\left(L / F_{\infty}\right)\right) \Lambda_{\mathfrak{r}} \subseteq A(T ; \theta, \psi) \Lambda_{\mathfrak{r}}
$$

i.e. every element of $\operatorname{Fitt}_{\Lambda_{\mathfrak{r}_{0}}}\left(\operatorname{Gal}\left(L / F_{\infty}\right)\right)$ is divisible by $A(T ; \theta, \psi)$ in $\Lambda_{\mathfrak{r}}$, and hence in $\Lambda_{\mathfrak{r}_{0}}$ also.

We next deduce the latter assertion from the former: There is a $\Lambda_{\mathfrak{r}_{0}}$-module $E$ of the form $\bigoplus_{i=1}^{m} \Lambda_{\mathfrak{r}_{0}} / P_{i}^{e_{i}}$ which is pseudo-isomorphic to $\operatorname{Gal}\left(L / F_{\infty}\right)$, where $P_{i}$ are prime ideals of $\Lambda_{\mathfrak{r}_{0}}$ of height one (the "elementary $\Lambda_{\mathfrak{r}_{0}}$-module associated with $\operatorname{Gal}\left(L / F_{\infty}\right)$ "). We thus obtain exact sequences of finitely generated torsion $\Lambda_{\mathfrak{r}_{0}}$-modules

$$
\left\{\begin{array}{l}
0 \rightarrow F_{1} \rightarrow E \rightarrow E^{\prime} \rightarrow 0 \\
0 \rightarrow E^{\prime} \rightarrow \operatorname{Gal}\left(L / F_{\infty}\right) \rightarrow F_{2} \rightarrow 0
\end{array}\right.
$$

with $F_{1}$ and $F_{2}$ finite.

Now from the properties 1, 5, 9 and 11 in the appendix of [11], respectively, we obtain:

$$
\left\{\begin{array}{l}
\operatorname{Fitt}_{\Lambda_{\mathrm{r}_{0}}}(E) \subseteq \operatorname{Fitt}_{\Lambda_{\mathrm{r}_{0}}}\left(E^{\prime}\right), \\
\operatorname{Fitt}_{\Lambda_{\mathfrak{r}_{0}}}(E)=\operatorname{char}_{\Lambda_{\mathfrak{r}_{0}}}(E)=\operatorname{char}_{\Lambda_{\mathfrak{r}_{0}}}\left(\operatorname{Gal}\left(L / F_{\infty}\right)\right), \\
\operatorname{Fitt}_{\Lambda_{\mathrm{r}_{0}}}\left(E^{\prime}\right) \cdot \operatorname{Fitt}_{\Lambda_{\mathfrak{r}_{0}}}\left(F_{2}\right) \subseteq \operatorname{Fitt}_{\Lambda_{\mathrm{r}_{0}}}\left(\operatorname{Gal}\left(L / F_{\infty}\right)\right), \\
\operatorname{Fitt}_{\Lambda_{\mathrm{r}_{0}}}\left(F_{2}\right) \supseteq(p, T)^{l} \quad \text { with } l=\operatorname{length}_{\Lambda_{\mathfrak{r}_{0}}}\left(F_{2}\right) .
\end{array}\right.
$$

Putting all these together, we conclude that

$$
\operatorname{char}_{\Lambda_{\mathbf{r}_{0}}}\left(\operatorname{Gal}\left(L / F_{\infty}\right)\right) \cdot(p, T)^{l} \subseteq \operatorname{Fitt}_{\Lambda_{\mathfrak{r}_{0}}}\left(\operatorname{Gal}\left(L / F_{\infty}\right)\right)
$$

from which our claim follows.

\section{A.2. Characteristic power series of $\operatorname{Gal}\left(L / F_{\infty}\right)$}

In this final subsection, we show that the "extra factor" appearing in $B(T ; \theta, \psi)$ corresponds to the ramification in $L / F_{\infty}$ of primes above $l$ such that $l \mid N$ and $l \nmid \operatorname{cond}(\xi)$, and we determine the characteristic power series of $\operatorname{Gal}\left(L / F_{\infty}\right)$, when $\xi \neq \omega^{-2}$.

We already know that the extension $L / F_{\infty}$ is unramified outside $N$. We fix a prime $l$ dividing $N$ in the following. There are only finitely many primes of $F_{\infty}$ above $l$, and let $\mathfrak{l}_{1}, \ldots, \mathfrak{l}_{m}$ be all such primes. Let $L(l)$ be the maximal subextension of $L / F_{\infty}$ in which all $\mathfrak{l}_{i}$ are unramified. This is a Galois extension of $\mathbf{Q}$. By class field theory, $\operatorname{Gal}(L / L(l))$ is isomorphic to a quotient of $\lim _{n} \prod_{\lambda \mid l} \mathfrak{o}_{F_{n}, \lambda}^{\times}$. Here, $F_{n}$ is the $n$th layer of the $\mathbf{Z}_{p}$-extension $F_{\infty} / F, \mathfrak{o}_{F_{n}, \lambda}$ is the $\lambda$-adic completion of the ring of integers of $F_{n}$, and the projective limit is relative to the norm mappings. Since $\operatorname{Gal}\left(L / F_{\infty}\right)$ is a pro- $p$ group, it is also isomorphic to a quotient of $\lim _{n} \prod_{\lambda \mid l} \mathfrak{k}\left(\mathfrak{o}_{F_{n}, \lambda}\right)^{\times}, \mathfrak{k}$ 
meaning the residue field. Again by class field theory, the action of $\operatorname{Gal}\left(F_{\infty} / \mathbf{Q}\right)$ on $\operatorname{Gal}(L / L(l))$ corresponds to the natural action on $\lim _{n} \prod_{\lambda \mid l} \mathfrak{k}\left(\mathfrak{o}_{F_{n}, \lambda}\right)^{\times}$. Considering the action of the inertia group of $l$ in $\Delta$, we see from (A.1.10) that $L=L(l)$ when $l \mid \operatorname{cond}(\xi)$. (This argument is due to Mazur and Wiles ([11], Chapter 1, Section 8).)

Lemma (A.2.1). - Assume that l divides $N$, but does not divide cond $(\xi)$. Then $\operatorname{Gal}(L / L(l))$ is a cyclic $\mathfrak{r}_{0} \llbracket \Gamma \rrbracket$-module annihilated by

$$
(1+T)^{s(l)}-(\xi \omega)(l) l=: b_{l}(T) \in \mathfrak{r}_{0} \llbracket \Gamma \rrbracket=\Lambda_{\mathfrak{r}_{0}} .
$$

Proof. - In this case, the $\operatorname{Gal}\left(F_{\infty} / \mathbf{Q}\right)$-module $\operatorname{Gal}\left(L / F_{\infty}\right)$ is unramified at $l$, by lemma (A.1.10). Moreover, the same lemma, after the "change of variable" $1+T \leftrightarrow u_{0}^{-1}(1+T)^{-1}$, implies that the Iwasawa theoretic action of a geometric Frobenius $\Phi_{l} \in \operatorname{Gal}\left(F_{\infty} / \mathbf{Q}\right)$ at $l$ is given by $(\xi \omega)(l)(1+T)^{-s(l)}$. On the other hand, $\Phi_{l}$ acts on $\lim _{n} \prod_{\lambda \mid l} \mathfrak{k}\left(\mathfrak{o}_{F_{n}, \lambda}\right)^{\times}$as multiplication by $l^{-1}$ (if we consider this group as an additive group). It follows that $\operatorname{Gal}(L / L(l))$ is annihilated by $(\xi \omega)(l)(1+T)^{-s(l)}-l^{-1}$, and hence by its unit multiple $b_{l}(T)$ also.

For $n$ large enough, the restrictions $\mathfrak{l}_{n, i}$ of $\mathfrak{l}_{i}$ to $F_{n}$ give $m$ distinct primes, and each $\lim _{n \gg 0} \mathfrak{k}\left(\mathfrak{o}_{F_{n}, \mathfrak{l}_{n, i}}\right) \times$ is a pro-cyclic group. Since $\operatorname{Gal}\left(F_{\infty} / \mathbf{Q}\right)$ acts transitively on $\left\{\mathfrak{l}_{1}, \ldots, \mathfrak{l}_{m}\right\}$, we conclude that $\operatorname{Gal}(L / L(l))$ is a cyclic module over $\mathbf{Z}_{p} \llbracket \Delta \times \Gamma \rrbracket$, and hence over $\Lambda_{\mathfrak{r}_{0}}$.

THEOREM (A.2.2). - Consider $\operatorname{Gal}\left(L / F_{\infty}\right)$ as a $\Lambda_{\mathfrak{r}_{0}}$-module Iwasawa theoretically. If $\xi \neq \omega^{-2}$, then we have

$$
\operatorname{char}_{\Lambda_{\mathfrak{r}_{0}}}\left(\operatorname{Gal}\left(L / F_{\infty}\right)\right)=B(T ; \theta, \psi) \Lambda_{\mathfrak{r}_{0}}
$$

Proof. - Let $l$ be a prime such that $l \mid N$ but $l \nmid \operatorname{cond}(\xi)$. From the exact sequence:

$$
0 \rightarrow \operatorname{Gal}(L / L(l)) \rightarrow \operatorname{Gal}\left(L / F_{\infty}\right) \rightarrow \operatorname{Gal}\left(L(l) / F_{\infty}\right) \rightarrow 0
$$

of $\Lambda_{\mathfrak{r}_{0}}$-modules and the lemma above we see that:

$$
\operatorname{char}_{\Lambda_{\mathfrak{r}_{0}}}\left(\operatorname{Gal}\left(L / F_{\infty}\right)\right) \supseteq b_{l}(T) \cdot \operatorname{char}_{\Lambda_{\mathfrak{r}_{0}}}\left(\operatorname{Gal}\left(L(l) / F_{\infty}\right)\right) .
$$

Let $L^{\text {ur }}$ be the maximal everywhere unramified subextension of $L / F_{\infty}$. Then repeating the argument above, we obtain

$$
\operatorname{char}_{\Lambda_{\mathfrak{r}_{0}}}\left(\operatorname{Gal}\left(L / F_{\infty}\right)\right) \supseteq\left(\prod_{\substack{l \mid N \\ l \nmid \operatorname{cond}(\xi)}} b_{l}(T)\right) \operatorname{char}_{\Lambda_{\mathfrak{r}_{0}}}\left(\operatorname{Gal}\left(L^{\mathrm{ur}} / F_{\infty}\right)\right)
$$

By (A.1.13), the left-hand side is contained in $B(T ; \theta, \psi) \Lambda_{\mathfrak{r}_{0}}$, and we note that this implies

$$
F\left(T, \xi \omega^{2}\right) \Lambda_{\mathfrak{r}_{0}} \supseteq \operatorname{char}_{\Lambda_{\mathfrak{r}_{0}}}\left(\operatorname{Gal}\left(L^{\mathrm{ur}} / F_{\infty}\right)\right) .
$$

However, since we assumed that $\xi \omega^{2} \neq 1$, the Iwasawa main conjecture proved by Mazur and Wiles [11] (cf. also [16]) assures us the reverse inclusion, i.e.:

$$
\operatorname{char}_{\Lambda_{\mathfrak{r}_{0}}}\left(\operatorname{Gal}\left(L / F_{\infty}\right)\right) \supseteq B(T ; \theta, \psi) \Lambda_{\mathfrak{r}_{0}} .
$$


During the course of the proof above, we have constructed large enough unramified extension $L^{\text {ur }}$ of $F_{\infty}$ using the Galois representation on $e^{*} E S_{p}(N)_{\mathfrak{r}, \mathfrak{m}^{*}}$ with $\mathfrak{m}^{*}=\mathfrak{m}^{*}(\theta, \psi)$. More precisely, for the same reason as [15], (5.3.20), we have:

COROllary (A.2.3). - Again assume that $\xi \neq \omega^{-2}$. Let $L_{\infty}$ be the maximal unramified pro-p abelian extension of $F_{\infty}$. Then $L^{\mathrm{ur}}$ above is the subextension of $L_{\infty} / F_{\infty}$ corresponding to the quotient $\operatorname{Gal}\left(L_{\infty} / F_{\infty}\right) \otimes \mathbf{z}_{p}[\Delta] \mathfrak{r}_{0}$ of the Galois group, where we consider the tensor product via $(\xi \omega)^{-1}: \Delta \rightarrow \mathfrak{r}_{0}^{\times}$.

In Section 3.2, we left a part of the proof of (1.5.5) in the case (II) unproved. The following corollary covers this lacuna:

COROllary (A.2.4). $-\mathfrak{h}^{*} / I^{*}$ is isomorphic to $\Lambda_{\mathfrak{r}} /(A(T ; \theta, \psi))$ as a $\Lambda_{\mathfrak{r}}$-algebra.

Proof. - We have proved this under the assumption that $\psi \neq 1$ in Section 3.2. Since

$$
(\theta, \psi) \neq\left(\omega^{-2}, \mathbf{1}\right)
$$

we may assume that $\xi \neq \omega^{-2}$. Consider $\operatorname{Gal}\left(L / F_{\infty}\right)$ as a $\Lambda_{\mathfrak{r}_{0}}$-submodule of $B / I^{*} B$. Then from the proof of (A.1.13) and (A.2.2), we have

$$
A(T ; \theta, \psi) \Lambda_{\mathfrak{r}} \supseteq \mathfrak{a} \supseteq \operatorname{Fitt}_{\Lambda_{\mathfrak{r}_{0}}}\left(\operatorname{Gal}\left(L / F_{\infty}\right)\right) \Lambda_{\mathfrak{r}} \supseteq A(T ; \theta, \psi)(p, T)^{l} \Lambda_{\mathfrak{r}}
$$

Our assertion follows from this, because we already know that $\mathfrak{a}$ is a principal ideal by (1.1.9).

\section{Corrections to [15]}

During the preparation of this paper, the author found errors in [15], and he would like to take this opportunity to correct them. These are caused by two misobservations: p. 256, lines 13-14 and p. 261, line 11, which are no longer true when $K$ is not finite over $\mathbf{Q}_{p}$. (A non-zero element of $\Lambda_{\mathfrak{o}}$ can have zeros of the form $\zeta u^{d}-1$ for infinitely many roots of unity $\zeta$ of $p$-power orders even for a fixed $d$; while the ring of integers of a finite extension of $K$ can be of infinite type as an o-module.) The proofs of (2.4.6) and (2.4.7) should be corrected as follows:

- They are valid when $K$ is finite over $\mathbf{Q}_{p}$. However, for the estimate of the rank given on p. 262, line 7 (in this case), we should have also appealed to the first statement in the proof of (2.4.6).

- In general, for a given $d \geqslant 0$ and a non-zero $F(T) \in \Lambda_{\mathfrak{o}}$, it is true that there are infinitely many characters $\varepsilon$ of $U_{1}$ such that $F\left(\varepsilon(u) u^{d}-1\right) \neq 0$. Assume that $K$ contains all the roots of unity of $p$-power orders and the $\varphi(N)$ th roots of unity for the moment. Then the proof of (2.4.6), given after p. 261, line 22, and the statement concerning the rank in (2.4.7) remain valid with the remark above, for such a $K$.

- Let $K$ be as above. Then, at the end of the proof of (2.4.6), we can choose $\left\{\mathcal{F}_{1}, \ldots, \mathcal{F}_{r}\right\}$ from $e M_{k}^{\prime}\left(N ; \Lambda_{\mathbf{z}_{p}}\right)$. Consequently, $e M_{k}^{\prime}\left(N ; \Lambda_{\mathfrak{o}}\right)=e M_{k}^{\prime}\left(N ; \Lambda_{\mathbf{z}_{p}}\right) \otimes_{\Lambda_{\mathbf{z}_{p}}} \Lambda_{\mathfrak{o}}$, and it is independent of $k \geqslant 2$. The equality given on p. 262 , line 12 also follows from this, because the right-hand side is contained in $(1 / D(T))\left(\Lambda_{\mathfrak{o}} \mathcal{F}_{1}+\cdots+\Lambda_{\mathfrak{o}} \mathcal{F}_{r}\right)$ with a non-zero $D(T) \in \Lambda_{\mathbf{z}_{p}}$.

- The validity of the statements (2.4.6) and (2.4.7) (and the above mentioned equality) for general $K$ follows from this. 


\section{REFERENCES}

[1] Greenberg R., Stevens G., $p$-adic $L$-functions and $p$-adic periods of modular forms, Invent. Math. 111 (1993) 407-447.

[2] Harder G., Pink R., Modular konstruierte unverzweigte abelsche $p$-Erweiterungen von $\mathbb{Q}\left(\zeta_{p}\right)$ und die Struktur ihrer Galoisgruppen, Math. Nachr. 159 (1992) 83-99.

[3] Hecke E., Theorie der Eisensteinschen Reihen höherer Stufe und ihre Anwendung auf Funktionentheorie und Arithmetik, Abh. Math. Sem. Hamburg 5 (1927) 199-224 (Math. Werke No. 24).

[4] HidA H., Iwasawa modules attached to congruences of cusp forms, Ann. Sci. Éc. Norm. Sup. (4) 19 (1986) 231-273.

[5] HidA H., Hecke algebras for $\mathrm{GL}_{1}$ and $\mathrm{GL}_{2}$, in: Sém. Théorie des Nombres, Paris, 1984-85, in: Progress in Math., Vol. 63, Birkhäuser, 1986, pp. 131-163.

[6] HidA H., Galois representations into $\mathrm{GL}_{2}\left(\mathbf{Z}_{p} \llbracket X \rrbracket\right)$ attached to ordinary cusp forms, Invent. Math. 85 (1986) 543-613.

[7] HidA H., A $p$-adic measure attached to the zeta functions associated with two elliptic modular forms II, Ann. Inst. Fourier 38 (1988) 1-83.

[8] HidA H., Elementary Theory of L-functions and Eisenstein Series, in: London Math. Soc. Stud. Texts, Vol. 26, Cambridge Univ. Press, 1993.

[9] KURIHARA M., Ideal class groups of cyclotomic fields and modular forms of level 1, J. Number Theory 45 (1993) 281-294.

[10] Kubert D., LANG S., Modular units, Springer-Verlag, 1981.

[11] Mazur B., Wiles A., Class fields of abelian extensions of Q, Invent. Math. 76 (1984) 179-330.

[12] Mazur B., Wiles A., On p-adic analytic families of Galois representations, Comp. Math. 59 (1986) 231-264.

[13] Онта M., On cohomology groups attached to towers of algebraic curves, J. Math. Soc. Japan 45 (1993) 131-183.

[14] Онта M., On the $p$-adic Eichler-Shimura isomorphism for $\Lambda$-adic cusp forms, J. Reine Angew. Math. 463 (1995) 49-98.

[15] Онта M., Ordinary $p$-adic étale cohomology groups attached to towers of elliptic modular curves, Comp. Math. 115 (1999) 241-301.

[16] Oнта M., Ordinary $p$-adic étale cohomology groups attached to towers of elliptic modular curves. II, Math. Ann. 318 (2000) 557-583.

[17] S ABy N., Théorie d'Iwasawa géométrique: un théorème de comparaison, J. Number Theory 59 (1996) 225-247.

[18] ShImURA G., Introduction to the Arithmetic Theory of Automorphic Functions, Iwanami Shoten and Princeton Univ. Press, 1971.

[19] Stevens G., Arithmetic on Modular Curves, in: Progress in Math., Vol. 20, Birkhäuser, 1982.

[20] Tilouine J., Un sous-groupe $p$-divisible de la jacobienne de $X_{1}\left(N p^{r}\right)$ comme module sur l'algèbre de Hecke, Bull. Soc. Math. Fr. 115 (1987) 329-360.

[21] WiLES A., On ordinary $\lambda$-adic representations associated to modular forms, Invent. Math. 94 (1988) 529-573.

[22] WiLES A., The Iwasawa conjecture for totally real fields, Ann. Math. 131 (1990) 493-540.

(Manuscrit reçu le 3 août 2001; accepté, après révision, le 21 mai 2002.)

\footnotetext{
Masami OHTA

Department of Mathematics,

Faculty of Science, Tokai University,

Hiratsuka, Kanagawa, 259-1292, Japan

E-mail: ohta@sm.u-tokai.ac.jp
} 